\title{
Improved effective-one-body description of coalescing nonspinning black-hole binaries and its numerical-relativity completion
}

\author{
Thibault Damour ${ }^{1}$, Alessandro Nagar ${ }^{1}$, and Sebastiano Bernuzzi ${ }^{2}$ \\ ${ }^{1}$ Institut des Hautes Etudes Scientifiques, 91440 Bures-sur-Yvette, France and \\ ${ }^{2}$ Theoretical Physics Institute, University of Jena, 07743 Jena, Germany
}

(Dated: October 30, 2018)

\begin{abstract}
We improve the effective-one-body (EOB) description of nonspinning coalescing black hole binaries by incorporating several recent analytical advances, notably: (i) logarithmic contributions to the conservative dynamics; (ii) resummed horizon-absorption contribution to the orbital angular momentum loss; and (iii) a specific radial component of the radiation reaction force implied by consistency with the azimuthal one. We then complete this analytically improved EOB model by comparing it to accurate numerical relativity (NR) simulations performed by the Caltech-CornellCITA group for mass ratios $q=(1,2,3,4,6)$. In particular, the comparison to NR data allows us to determine with high-accuracy $\left(\sim 10^{-4}\right)$ the value of the main EOB radial potential: $A(u ; \nu)$, where $u=G M /\left(R c^{2}\right)$ is the inter-body gravitational potential and $\nu=q /(q+1)^{2}$ is the symmetric mass ratio. We introduce a new technique for extracting from $\mathrm{NR}$ data an intrinsic measure of the phase evolution, $\left(Q_{\omega}(\omega)\right.$ diagnostics). Aligning the NR-completed EOB quadrupolar waveform and the $\mathrm{NR}$ one at low frequencies, we find that they keep agreeing (in phase and amplitude) within the NR uncertainties throughout the evolution for all mass ratios considered. We also find good agreement for several subdominant multipoles without having to introduce and tune any extra parameters.
\end{abstract}

\section{INTRODUCTION}

The Effective One Body (EOB) formalism [1-5] has been proposed as a new analytical method for describing the motion and radiation of coalescing black hole binaries. One of its main aims is to provide analytical ${ }^{1}$ gravitational wave $(\mathrm{GW})$ templates covering the full coalescence process, from early inspiral to ringdown, passing through late inspiral, plunge and merger. The definition of the EOB formalism mainly relies on two sources of information:

(i) high-order results of post-Newtonian (PN) theory;

(ii) high-accuracy results from Numerical Relativity (NR) simulations of coalescing black hole binaries (both in the comparable-mass case, $\nu=\mathcal{O}(1)$, and in the extreme-mass-ratio limit, $\nu \ll 1)$. [Here, $\nu \equiv$ $m_{1} m_{2} /\left(m_{1}+m_{2}\right)^{2}$ denotes the symmetric mass ratio.]

In addition, EOB theory has recently tapped useful information out of Gravitational Self Force (GSF) computations at order $\mathcal{O}(\nu)$. All this information is not used in its original form, but rather as a way to determine, or at least constrain, the structure of the few basic functions that enter the definition of the EOB formalism. For recent general reviews of the EOB formalism and its historical roots, see $[6,7]$.

The EOB formalism has been developed in a sequence of papers, both for nonspinning black hole binaries [1$3,5,8,9]$ and for spinning ones [4, 10-14]. In addition, it has been extended to the case of tidally interacting

\footnotetext{
${ }^{1}$ Here we use the adjective "analytical" (instead of "semianalytical") for methods that are based on solving analytically given ordinary differential equations, even if one needs to use numerical tools to solve them.
}

neutron star binaries $[15,16]$. For all those types of systems, many comparisons between the predictions of $\mathrm{EOB}$ theory and the results of NR simulations have been performed $[9,17-29]$ and have demonstrated that it is possible to devise accurate EOB waveforms by combining improved resummation methods [5, 8, 9], high-order $\mathrm{PN}$ results (see [30] for a review), and some nonperturbative information coming from high-accuracy NR results. These $\mathrm{EOB}$ waveforms can be used both in GW detection and in GW parameter-estimation protocols. The EOB formalism can thereby crucially help detecting the GWs emitted by coalescing black hole binaries, since many thousands of waveform templates need to be computed to extract the signal from the broad-band noise, an impossible task for NR alone. The EOB formalism might also be crucial in allowing one to extract information on the equation of state of nuclear matter from observations of coalescing neutron star binaries [31]. An early version of the EOB waveform [28] has already been incorporated ${ }^{2}$, and used [32] in the LIGO and Virgo search pipeline.

In addition, some recent comparisons between NR studies of the dynamics of black hole binaries and its EOB description, have directly confirmed the ability of EOB theory to accurately describe several (gauge-invariant) aspects of the conservative dynamics of binary systems, such as periastron precession [33] and the relation between energy and angular momentum [26].

The aim of the present paper is to improve the definition of some of the basic elements of the EOB formalism both by including for the first time recently obtained analytical information, and by extracting, in a new way, nonperturbative information from accurate NR simulations performed by the Caltech-Cornell-CITA group [34].

\footnotetext{
${ }^{2}$ See https://www.lsc-group.phys.uwm.edu/daswg/projects/lalsuite.html.
} 
Though our study will be limited to nonspinning binaries, the EOB structures we shall improve (such as the basic EOB radial potential $A(R)$ ) are central, and should then be included both in the spinning and tidal extensions of the EOB formalism.

The recent analytical progresses that we shall incorporate here in EOB theory are:

(i) $4 \mathrm{PN}$ and $5 \mathrm{PN}$ logarithmic contributions to the conservative dynamics [35-38];

(ii) the $\mathcal{O}(\nu) 4 \mathrm{PN}$ nonlogarithmic contribution to the conservative dynamics [36, 38-40];

(iii) resummed horizon-absorption contributions to angular momentum loss [41, 42];

(iv) the radial component of the radiation reaction force implied by consistency with the azimuthal one [43];

(v) an additional $3.5 \mathrm{PN}$ contribution to the phase of the (factorized [5, 8, 9]) quadrupolar waveform [44].

In addition, we shall bring up some novelties in the definition of the EOB formalism, and in the way to extract information from (comparable-mass) NR data. Namely:

(a) we introduce a Padé resummation of the additional tail phases $\delta_{\ell m}$ of the factorized EOB waveform;

(b) we show how to accurately extract from NR data the $Q_{\omega}(\omega)$ function measuring, in an intrinsic way, the phase evolution of the (curvature) quadrupolar waveform;

(c) we introduce a new way to improve the EOB waveform during plunge and merger by matching it to the NR one at a specifically chosen ( $\nu$-dependent) NR time $t_{\text {extr }}^{\mathrm{NR}}(\nu)$ around merger. More precisely, we impose [by using six next-to-quasi-circular (NQC) parameters] a $C^{2}$ contact condition between the amplitudes and the frequencies of the NR and EOB waveforms at an NR instant $t_{\text {extr }}^{\mathrm{NR}}(\nu)$, corresponding to the maximum of the $\mathrm{EOB}$ orbital frequency $t_{\Omega \text { peak }}^{\text {EOB }}$.

The paper is organized as follows. In Sec. II we present, in a self contained manner, the detailed definition of our improved EOB formalism. Section III explains how to extract the $Q_{\omega}(\omega)$ function from NR data while Sec. IV revisits the extreme-mass-ratio case. In Sec. V we then complete our new EOB formalism by comparison with several comparable-mass simulations performed by the Caltech-Cornell-CITA group. Section VI studies the structure of the main EOB radial potential $(A(u)$ function) obtained from the latter NR comparison and Sec. VII discusses how to compute EOB waveforms for arbitrary values of $\nu$. We summarize our main conclusions in Sec. VIII, while some supplemental material is presented in several Appendixes. In particular, Appendix D gives the explicit expressions of the $\rho_{\ell m}$ and $\delta_{\ell m}$ bricks of the EOB factorized waveform we use.

\section{EFFECTIVE-ONE-BODY ANALYTICAL FRAMEWORK}

In this section we shall present in detail the definition of the new (nonspinning) EOB formalism, incorporating several recent analytical improvements that we shall use in this paper. Our presentation will be self-contained so as to allow readers to generate for themselves all our EOB results. We also intend to make available soon a public version of our EOB codes.

The EOB formalism is made of three basic building blocks: (i) a EOB Hamiltonian that resums the conservative two-body dynamics; (ii) a resummed EOB radiation reaction force that completes the conservative dynamics by causing the system to inspiral down to merger, and (iii) a resummed EOB inspiral-plus-plunge waveform, together with a prescription for extending the waveform through merger and ringdown. Each one of these building blocks has been developed in previous papers. In particular, the construction of the EOB Hamiltonian was initiated in Refs. [1, 3], while the definition of the resummed, factorized inspiral waveform was initiated in Refs. [5, 8, 9]. Here we bring new (recently derived) theoretical improvements to each element of the formalism, namely: (i) we include logarithmic contributions [35-38] to the EOB Hamiltonian; (ii) we include the effect of a resummed version of horizon absorption [41, 42] in the radiation reaction; (iii) we add a recently derived [43] radial component of radiation reaction; (iv) we include the $3.5 \mathrm{PN}$ contribution [44] to the phase $\delta_{22}$ of the factorized quadrupolar waveform; (v) we resum $\delta_{22}$, as well as some higher-multipoles $\delta_{\ell m}$ 's, by Padé methods. All these improvements either add some new physics that was not included in the previous EOB models [22, 28], or improve [in the case of $(\mathrm{v})$ ] the robustness of the EOB resummations. We shall discuss them in detail in the sections below.

\section{A. Improved Hamiltonian: logarithmic contributions to the A function}

The conservative (nonspinning) two-body dynamics is described, within the EOB formalism, by a Hamiltonian $H_{\mathrm{EOB}}\left(Q^{i}, P_{i}\right)$, describing the relative motion $Q^{i}=$ $Q_{1}^{i}-Q_{2}^{i}$ of the binary, and depending on two radial functions, $A(R)$ and $B(R)$, where $R \equiv\left|Q^{i}\right|$ is the binary separation (in EOB coordinates). We are using phase space variables $\left(R, P_{R}, \varphi, P_{\varphi}\right)$ associated to polar coordinates in the equatorial plane $\theta=\pi / 2$. Actually it is useful to replace the radial momentum $P_{R}$ by the momentum $P_{R_{*}}=(A / B)^{1 / 2} P_{R}$ conjugate to the "tortoise" radial coordinate $R_{*}=\int d R(B / A)^{1 / 2}$. Furthermore, it is convenient to use suitably rescaled dimensionless variables:

$$
r=\frac{R}{G M}, p_{r_{*}}=\frac{P_{R_{*}}}{\mu}, p_{\varphi}=\frac{P_{\varphi}}{\mu G M}, t=\frac{T}{G M} .
$$


Here, and in the following, we use the notation

$$
M \equiv m_{1}+m_{2}, \mu \equiv \frac{m_{1} m_{2}}{m_{1}+m_{2}}, \nu \equiv \frac{\mu}{M}, q \equiv \frac{m_{1}}{m_{2}} .
$$

Note that the dimensionless symmetric mass ratio $\nu=$ $m_{1} m_{2} /\left(m_{1}+m_{2}\right)^{2}=q /(q+1)^{2}$ varies between 0 (extreme mass-ratio case) and $\frac{1}{4}$ (equal-mass case), and that we shall conventionally consider that $m_{2} \leq m_{1}$, so that $q \geq$ 1 . In addition we generally set $c=1$, and shall also often set $G=1$ in the following.

With the above notation, the $\mu$-rescaled (real) EOB Hamiltonian reads

$$
\hat{H}_{\mathrm{EOB}}\left(r, p_{r_{*}}, p_{\varphi}\right) \equiv \frac{H_{\mathrm{EOB}}}{\mu}=\frac{1}{\nu} \sqrt{1+2 \nu\left(\hat{H}_{\mathrm{eff}}-1\right)},
$$

where $\hat{H}_{\text {eff }}$ denotes the ( $\mu$-rescaled) effective EOB Hamiltonian, given by

$$
\hat{H}_{\mathrm{eff}}=\sqrt{p_{r_{*}}^{2}+A(r)\left(1+\frac{p_{\varphi}^{2}}{r^{2}}+z_{3} \frac{p_{r_{*}}^{4}}{r^{2}}\right)},
$$

with $z_{3}=2 \nu(4-3 \nu)$.

The (rescaled) EOB Hamiltonian (3) leads to equations of motion for $\left(r, \varphi, p_{r_{*}}, p_{\varphi}\right)$ with respect to the rescaled time $t=T / G M$, Eq. (1), of the form ${ }^{3}$

$$
\begin{aligned}
\frac{d \varphi}{d t} & \equiv \Omega=\frac{\partial \hat{H}_{\mathrm{EOB}}}{\partial p_{\varphi}}, \\
\frac{d r}{d t} & =\left(\frac{A}{B}\right)^{1 / 2} \frac{\partial \hat{H}_{\mathrm{EOB}}}{\partial p_{r_{*}}}, \\
\frac{d p_{\varphi}}{d t} & =\hat{\mathcal{F}}_{\varphi}, \\
\frac{d p_{r_{*}}}{d t} & =-\left(\frac{A}{B}\right)^{1 / 2} \frac{\partial \hat{H}_{\mathrm{EOB}}}{\partial r}+\hat{\mathcal{F}}_{r_{*}},
\end{aligned}
$$

which explicitly read

$$
\begin{aligned}
& \frac{d \varphi}{d t} \equiv \Omega=\frac{A p_{\varphi}}{\nu r^{2} \hat{H}_{\mathrm{EOB}} \hat{H}_{\mathrm{eff}}} \\
& \frac{d r}{d t}=\left(\frac{A}{B}\right)^{1 / 2} \frac{1}{\nu \hat{H}_{\mathrm{EOB}} \hat{H}_{\mathrm{eff}}}\left(p_{r_{*}}+z_{3} \frac{2 A}{r^{2}} p_{r_{*}}^{3}\right) \\
& \frac{d p_{\varphi}}{d t}=\hat{\mathcal{F}}_{\varphi} \\
& \frac{d p_{r_{*}}}{d t}=-\left(\frac{A}{B}\right)^{1 / 2} \frac{1}{2 \nu \hat{H}_{\mathrm{EOB}} \hat{H}_{\mathrm{eff}}} \\
& \left\{A^{\prime}+\frac{p_{\varphi}^{2}}{r^{2}}\left(A^{\prime}-\frac{2 A}{r}\right)+z_{3}\left(\frac{A^{\prime}}{r^{2}}-\frac{2 A}{r^{3}}\right) p_{r_{*}}^{4}\right\}+\hat{\mathcal{F}}_{r_{*}}
\end{aligned}
$$

\footnotetext{
${ }^{3}$ For clarity, we shall sometimes restore the $M$ 's in the text below, as well as in the figures.
}

where $A^{\prime}=d A / d r$. In these equations, $\hat{\mathcal{F}} \equiv \mathcal{F} / \mu$ denotes the $\mu$-rescaled radiation-reaction force. Its explicit form will be given in Sec. IID below.

Let us now define the explicit forms of the two basic EOB radial functions $A(r)$ and $B(r)$ entering the Hamiltonian (3). One of the main theoretical novelties of the EOB model used in the present work is the inclusion in $A(r)$ (which plays the role of the main radial potential in the EOB Hamiltonian) of the recently computed logarithmic contributions appearing at the $4 \mathrm{PN}$ and $5 \mathrm{PN}$ levels [35-38]. If we first focus on the Taylor-expanded version of the $A$ potential, it has, when considered at the $5 \mathrm{PN}$ level, the form

$$
\begin{aligned}
& A^{\text {Taylor }}(u)=1-2 u+2 \nu u^{3}+\left(\frac{94}{3}-\frac{41}{32} \pi^{2}\right) \nu u^{4} \\
& +\nu\left[a_{5}^{c}(\nu)+a_{5}^{\ln }(\nu) \ln u\right] u^{5}+\nu\left[a_{6}^{c}(\nu)+a_{6}^{\ln }(\nu) \ln u\right] u^{6},
\end{aligned}
$$

where $u \equiv G M / R \equiv 1 / r$ denotes the (EOB) dimensionless gravitational potential, and where

$$
\begin{aligned}
& a_{5}^{\ln }(\nu)=\frac{64}{5}, \\
& a_{6}^{\ln }(\nu)=-\frac{7004}{105}-\frac{144}{5} \nu,
\end{aligned}
$$

denote the analytically known logarithmic contributions, while $a_{5}^{c}(\nu)$ and $a_{6}^{c}(\nu)$ represent currently unknown, nonlogarithmic $\nu$-dependent $4 \mathrm{PN}$ and $5 \mathrm{PN}$ contributions to $A(u)$. Following the EOB methodology initiated in Ref. [3], we do not use the Taylor-expanded radial potential $A^{\text {Taylor }}(u)$ to define the EOB Hamiltonian, but use instead its $(1,5)$ Padé approximant, namely

$$
\begin{aligned}
& A\left(u ; a_{5}^{c}(\nu), a_{6}^{c}(\nu) ; \nu\right) \equiv P_{5}^{1}\left[A^{\text {Taylor }}(u)\right] \\
& =\frac{1+n_{1} u}{1+d_{1} u+d_{2} u^{2}+d_{3} u^{3}+d_{4} u^{4}+d_{5} u^{5}},
\end{aligned}
$$

where the coefficients $n_{1}$ and $d_{i}$ appearing in the numerator and the denominator of the Padé approximant depend rationally on $a_{5}^{c}, a_{6}^{c}, \nu$ and $\ln u$.

As is well known, Padé approximants can sometimes exhibit "spurious poles" in $u$. The appearance of such poles was emphasized by Pan et al. [45] within the context of an EOB model for spinning black holes where the $A(u)$ radial potential is defined by Padéing a Taylor-expanded $A$ function augmented by Kerr-like spin-dependent terms (as suggested in Ref. [4]). In the case we shall investigate here (with $a_{5}^{c}$ fixed to the value in Eq. (13) below) we found that such a spurious pole is present even in the absence of spin, but that it is always located behind a horizon (i.e. a zero of $A(u)$ ). However, when $\nu=0.25$ and $a_{6}^{c} \lesssim-130$, the presence of this pole (even "hidden" behind the horizon) starts visibly affecting the position of the adiabatic light-ring (i.e. the location of the maximum of $\left.u^{2} A(u)\right)$, and thereby the 
late-plunge dynamics ${ }^{4}$. This hidden pole will not affect our analysis below because we shall work in the range $a_{6}^{c} \geq-110$. We note in this respect that the presence of spurious poles in the context of a spinning EOB model has motivated Barausse and Buonanno [12] to propose a different resummation of the $A$ potential which does not rely on Padé approximants, but imposes by hand the presence of a horizon.

The logarithmic-dependent 5PN-Padé-resummed radial potential $A\left(u ; a_{5}^{c}, a_{6}^{c} ; \nu\right)$ will play in our work the role played by the nonlogarithmic $5 \mathrm{PN}$ Padéed potentials $A^{\text {no- } \log }\left(u ; a_{5}, a_{6} ; \nu\right)$ (obtained by replacing $a_{5}^{c}(\nu)+$ $a_{5}^{\ln }(\nu) \ln u \rightarrow a_{5}(\nu)$ and $a_{6}^{c}(\nu)+a_{6}^{\ln }(\nu) \ln u \rightarrow a_{6}(\nu)$ in the formulas above) used in the previous EOB works $[6$, $22,28]$. As in those references, we shall use NR data to constrain, for each value of the symmetric mass ratio $\nu$, the values of $a_{5}^{c}(\nu)$, and $a_{6}^{c}(\nu)$. To simplify this task, we shall take into account from the beginning a finding of Refs. [6, 22, 28]. The latter references found that there is, for each value of $\nu$, a good EOB/NR agreement within a long and thin banana-like region in the $\left(a_{5}, a_{6}\right)$ plane. In view of this degeneracy between $a_{5}$ and $a_{6}$, we shall then fix the value of $a_{5}^{c}$ and fit only for the ( $\nu$-dependent) value of $a_{6}^{c}(\nu)$.

Recent works connecting $\mathrm{PN}$ and/or EOB theory to gauge-invariant observables computable from GSF theory have succeeded in determining the $\nu$-linear contributions to the two EOB potentials $A(u ; \nu)$ and $B(u ; \nu)[35$, $38,40,46-48]$. In particular, the limiting values as $\nu \rightarrow 0$ of the Taylor value of $a_{5}^{c}(\nu)$ and $a_{6}^{c}(\nu)$ (defined from Eq. (7)) were found by Barausse et al. [38] to be $a_{5}^{c \text { Taylor }}(0)=23.50190(5)$ and $a_{6}^{c \text { Taylor }}(0)=-131.72(1)$. It is important to note here that these values correspond to the "true" Taylor coefficients of the PN-expansion of the $A(u)$ function when $u \rightarrow 0$, i.e., the coefficients of $u^{5}$ and $u^{6}$ in an expansion in powers of $u$ around $u=0^{5}$.

However, within our present EOB model the meaning of the parameters $\left(a_{5}^{c}(\nu), a_{6}^{c}(\nu)\right)$ is different. First, when $\nu \rightarrow 0$, as the expansion (7) does not include powers of $u$ beyond $u^{6}$, any attempt at determining values of $\left(a_{5}^{c}(0), a_{6}^{c}(0)\right)$ by comparing Eq. (7) to GSF data will strongly depend on the $u$-interval where this comparison is done. For instance, we might want to require that the function $\mathrm{a}(u)[35]$ takes at $u=1 / 6$, i.e. at the unperturbed $\nu=0 \mathrm{LSO}$, the numerical value corresponding to periastron precession, as determined by GSF calculations [48, 49]. This would lead (similarly to what is done in Ref. [35] which did not take into account logarithmic contributions) to determining values of $\left(a_{5}^{c}(0), a_{6}^{c}(0)\right)$

\footnotetext{
${ }^{4}$ By contrast, for $\nu \lesssim 0.2$ the spurious pole still exists but has nearly no effect neither on the location of the adiabatic lightring nor on the late-plunge dynamics.

${ }^{5}$ By contrast, note that Ref. [48] obtained slightly different values of $a_{5}^{c}(0)$ and $a_{6}^{c}(0)$, namely $a_{5}^{c \text { eff }}(0)=23.47267$ and $a_{6}^{c \text { eff }}(0)=$ -127.154 , because they were derived from $u$-global fits instead of an expansion around $u=0$.
}

such that Eq. (92) of [48], namely

$$
\mathrm{a}\left(1 / 6, a_{5}^{c}, a_{6}^{c}\right)=0.795883004(15)
$$

is satisfied. Taking for instance $a_{5}^{c}(0)=23.50190(5)$ [38], we would then get the following "effective" value of $a_{6}^{c}(0)$

$$
a_{6}^{c}(0)=+39.1223 \quad \text { [from GSF LSO precession]. }
$$

Note that this value is completely different, even in sign, from the value $a_{6}^{c \text { Taylor }}(0)=-131.72(1)$ which refers to the Taylor expansion around $u=0$.

A second reason why the meaning of $\left(a_{5}^{c}(\nu), a_{6}^{c}(\nu)\right)$ is different in our framework than in the GSF one is that the function $A\left(u ; a_{5}^{c}(\nu), a_{6}^{c}(\nu) ; \nu\right)$ defined by Eq. (10) is the Padé-resummed version of the Taylor polynomial given in Eq. (7), which does not contain any term beyond $u^{6}$. This implies that, when $\nu \neq 0$, the Taylor-expansion of $A\left(u ; a_{5}^{c}(\nu), a_{6}^{c}(\nu) ; \nu\right)$ does contain higher-order terms in $u$ which are all expressed in terms of $\left(a_{5}^{c}, a_{6}^{c}\right)$ and $\nu$. Therefore, the values of $\left(a_{5}^{c}(\nu), a_{6}^{c}(\nu)\right)$ extracted by comparison with NR data (for $\nu \neq 0$ ) represent a kind of mix between the true Taylor values and a plethora of higherorder $\mathrm{PN}$ corrections. In other words $\left(a_{5}^{c}(\nu), a_{6}^{c}(\nu)\right)$ represent an effective parametrization of the global shape of the $A$ potential.

Summarizing, in view of the effective character of the parameters $\left(a_{5}^{c}(\nu), a_{6}^{c}(\nu)\right)$ there is no necessity to impose that their $\nu \rightarrow 0$ limits coincide with those of Ref. [38]. However, due to the strong degeneracy between $\left(a_{5}^{c}(\nu), a_{6}^{c}(\nu)\right)$, it is convenient to fix $a_{5}^{c}(\nu)$ to some fiducial value. We then decided to use the following simple, $\nu$-independent, fiducial value

$$
a_{5}^{c \text { fiducial }}(\nu)=23.5 \text {. }
$$

We will see later that we could have replaced this value (which is compatible with the rounded-off Taylor value of $\left.a_{5}^{c}(0)\right)$ with a significantly different one.

Finally, as the other EOB potential $B(u)$, or equivalently the associated potential

$$
D(u ; \nu) \equiv A(u ; \nu) B(u, \nu),
$$

plays only a secondary role in the dynamics of coalescing binaries, and is therefore difficult to probe by using NR data, we used its 3PN-resummed value as obtained in Ref. [3], namely

$$
D(u ; \nu)=\frac{1}{1+6 \nu u^{2}+2(23-3 \nu) \nu u^{3}},
$$

without trying to improve it by including the known logarithmic contributions appearing at $4 \mathrm{PN}$ and $5 \mathrm{PN}[37,38]$ (which mix with unknown nonlogarithmic contributions).

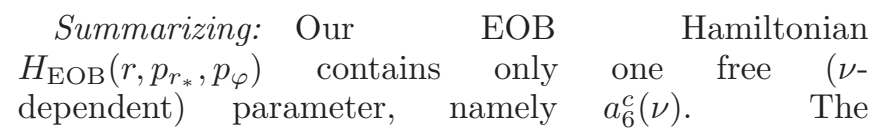
Hamiltonian $H_{\mathrm{EOB}}\left(r, p_{r_{*}}, p_{\varphi} ; a_{6}^{c}(\nu)\right)$ is defined by Eqs. (3), (4), (10), (15), with Eqs. (7), (8), (9), and (13), together with $p_{r_{*}}=(A / B)^{1 / 2} p_{r}, B(u) \equiv D(u) / A(u)$ and $u \equiv 1 / r$. 


\section{B. Improved EOB waveform during inspiral and plunge}

Following Refs. [5, 8, 9], we describe the inspiral-plusplunge multipolar waveform by the factorized structure

$$
h_{\ell m}^{\text {insplunge }}=h_{\ell m}^{(N, \epsilon)}\left(v_{\varphi}\right) S_{\text {eff }}^{(\epsilon)} \hat{h}_{\ell m}^{\text {tail }}(y)\left[\rho_{\ell m}\left(v_{\varphi}^{2}\right)\right]^{\ell} \hat{h}_{\ell m}^{\mathrm{NQC}},
$$

where we indicated the (main) arguments used in several factors of the waveform. Here $\epsilon=0,1$ is the parity of the considered multipole (i.e. the parity of $\ell+m), h_{\ell m}^{(N, \epsilon)}$ the Newtonian waveform, $\hat{S}_{\text {eff }}^{(\epsilon)}$ a source factor, with $\hat{S}_{\text {eff }}^{(0)}=$ $\hat{H}_{\text {eff }}$ or $\hat{S}_{\text {eff }}^{(1)}=p_{\varphi} /\left(r_{\omega} v_{\varphi}\right)$ according to the parity of the multipole (see below for definitions),

$$
\hat{h}_{\ell m}^{\text {tail }}(y) \equiv T_{\ell m}(y) e^{i \delta_{\ell m}(y)},
$$

the tail factor $[5,8,9], \rho_{\ell m}$ the resummed modulus correction and $\hat{h}_{\ell m}^{\mathrm{NQC}}$ a next-to-quasi-circular correction. The precise definitions of the factors entering Eq. (16), and of their arguments is given next.

The Newtonian contribution reads

$$
h_{\ell m}^{(N, \epsilon)}\left(v_{\varphi}\right)=\frac{M \nu}{R} n_{\ell m}^{(\epsilon)} c_{\ell+\epsilon}(\nu) v_{\varphi}^{\ell+\epsilon} Y^{\ell-\epsilon,-m}\left(\frac{\pi}{2}, \varphi\right),
$$

where $\varphi$ is the orbital phase, $v_{\varphi}=r_{\omega} \Omega$ a suitably defined azimuthal velocity, and $r_{\omega} \equiv r \psi^{1 / 3}$ a modified EOB radius with $\psi$ defined as

$$
\begin{aligned}
\psi\left(r, p_{\varphi}\right) & =\frac{2}{r^{2}}\left(\frac{d A}{d r}\right)^{-1} \\
& \times\left[1+2 \nu\left(\sqrt{A\left(1+\frac{p_{\varphi}^{2}}{r^{2}}\right)}-1\right)\right] .
\end{aligned}
$$

The definitions of $v_{\varphi}$ and $r_{\omega}$ are such that they satisfy Kepler's law, $1=\Omega^{2} r_{\omega}^{3}=v_{\varphi}^{2} r_{\omega}$, during the adiabatic inspiral [50]. In Eq. (18), $n_{\ell m}^{(\epsilon)}$ and $c_{\ell+\epsilon}(\nu)$ are numerical coefficients given by [5]

$$
\begin{aligned}
& n_{\ell m}^{(0)}=(i m)^{\ell} \frac{8 \pi}{(2 \ell+1) ! !} \sqrt{\frac{(\ell+1)(\ell+2)}{\ell(\ell-1)}} \\
& n_{\ell m}^{(1)}=-(i m)^{\ell} \frac{16 \pi i}{(2 \ell+1) ! !} \sqrt{\frac{(2 \ell+1)(\ell+2)\left(\ell^{2}-m^{2}\right)}{(2 \ell-1)(\ell+1) \ell(\ell-1)}} \\
& c_{\ell+\epsilon}=X_{2}^{\ell+\epsilon-1}+(-1)^{m} X_{1}^{\ell+\epsilon-1}
\end{aligned}
$$

where $X_{1,2} \equiv m_{1,2} / M$. [Note that, in our EOB/NR comparisons below, we shall often work with a "Zerilli-normalized" waveform, denoted $\Psi_{\ell m}$, whose normalization differs from that of $h_{\ell m}$ by a factor $R /(M \sqrt{(l+2)(l+1)(l)(l-1)}) \cdot]$ For what concerns the tail factor $\hat{h}_{\ell m}^{\text {tail }}$, Eq. (17), its main contribution, $T_{\ell m}$, is written as

$$
T_{\ell m}(y)=\frac{\Gamma(\ell+1-2 \mathrm{i} \hat{\hat{k}})}{\Gamma(\ell+1)} e^{\pi \hat{\hat{k}}} e^{2 \mathrm{i} \hat{\hat{k}} \ln \left(2 k r_{0}\right)},
$$

with $\hat{\hat{k}} \equiv m G H_{\mathrm{EOB}} \Omega, k \equiv m \Omega$ and $r_{0}=2 G M / \sqrt{e}$. Note that, apart from the $\operatorname{logarithm}$ term $\ln \left(2 k r_{0}\right)$, the main tail contribution $T_{\ell m}$ depends on the dimensionless argument $y \equiv\left(G H_{\mathrm{EOB}} \Omega\right)^{2 / 3}$, which differs from the usual dimensionless frequency parameter $x \equiv(G M \Omega)^{2 / 3}$ by the replacement $M \rightarrow H_{\mathrm{EOB}}$.

\section{Further resummation of the residual tail phase $\delta_{\ell m}(y)$.}

The main factorized tail term $T_{\ell m}(y)=\left|T_{\ell m}\right| e^{i \tau_{\ell m}}$ is a complex quantity whose modulus $\left|T_{\ell m}\right|$ describes the tail amplification of the waveform modulus, and whose phase $\tau_{\ell m}$ describes the main part of the dephasing caused by tails. There are, however, additional dephasings caused by tails, which are described by the supplementary phase factor $e^{i \delta_{\ell m}}$ in Eq. (17). The residual phase corrections $\delta_{\ell m}(y)$ entering the tail factor (17) were obtained in Ref. [5] as a PN series in the variable $y=\left(G H_{\mathrm{EOB}} \Omega\right)^{2 / 3}$. Here we shall use for $\delta_{\ell m}(y)$ an expression that differs both from the one originally given in Ref. [5], and from its test-mass-higher-PN completion given in Ref. [51]. More precisely: (i) we do not include the highest-order $O\left(y^{9 / 2}\right)$ test-mass $(\nu=0) \mathrm{PN}$ corrections because of their PN-gap with respect to the last known comparable-mass terms; (ii) we include the $3.5 \mathrm{PN}, \nu$-dependent, contribution to $\delta_{22}(y)$ that can be deduced from a recent analytical computation of the PN-expanded waveform at 3.5PN accuracy [44]; and (iii) we Padé-resum the Taylor series in powers of $y^{1 / 2}$ giving $\delta_{\ell m}(y)$. Indeed, we found that the PN-expanded version of $\delta_{\ell m}(y)$ presents some unpleasant features (discussed below in the $\ell=m=2$ case) that are avoided if one resums $\delta_{\ell m}\left(y^{1 / 2}\right)$ by factorizing the leading-order term and and replacing the rest with a suitable Padé approximant $N\left(y^{1 / 2}\right) / D\left(y^{1 / 2}\right)$.

Let us explain our new procedure on the (most important) example of the $\ell=m=2$ phase (the others are listed in Appendix D). Let us start from its Taylorexpanded form

$$
\begin{aligned}
\delta_{22}^{\text {Taylor }}(y) & =\frac{7}{3} y^{3 / 2}-24 \nu y^{5 / 2}+\frac{428}{105} \pi y^{3} \\
& +\left(\frac{30995}{1134} \nu+\frac{962}{135} \nu^{2}\right) y^{7 / 2}
\end{aligned}
$$

Here we did not include the highest-order test-mass term $\left(-2203 / 81+1712 / 315 \pi^{2}\right) y^{9 / 2}$ that was obtained in Ref. [51]. On the other hand, the 3.5 PN $\nu$-dependent term proportional to $y^{7 / 2}$ is a new contribution that is obtained by applying the factorization of [5] to the results of [44]. Note that this is the only genuinely new information given by this calculation; indeed, the real $3.5 \mathrm{PN}$ contributions to $h_{22}$ are already contained in the modulus of the EOB-resummed tail factor $\hat{h}_{\ell m}^{\text {tail }}$. For the comparable-mass cases that are of primary concern for upcoming GW observations (say for $\nu \gtrsim 0.1$ ) the $O\left(y^{7 / 2}\right.$ ) contribution is numerically quite significant compared to the lower-order terms. To better appreciate the relative 
importance of the successive PN corrections we factorize Eq. (24) in a leading order (LO) part, $\delta_{22}^{\mathrm{LO}}(y) \equiv(7 / 3) y^{3 / 2}$ and a fractional PN-correction term, $\hat{\delta}_{22} \equiv \delta_{22}^{\text {Taylor }} / \delta_{22}^{\mathrm{LO}}$. In terms of $v_{y} \equiv \sqrt{y}$, the latter fractional PN-correction has the structure

$$
\hat{\delta}_{22}=1+c_{2} v_{y}^{2}+c_{3} v_{y}^{3}+c_{4} v_{y}^{4} .
$$

We plot, in Fig. 1 the successive truncated PN approximants, at $1 \mathrm{PN}, 1.5 \mathrm{PN}$ and $2 \mathrm{PN}$ accuracy (i.e. up to $v_{y}^{2}$, $v_{y}^{3}$ and $\left.v_{y}^{4}\right)$ for $q=1(\nu=1 / 4)$ and $q=6(\nu=6 / 49 \approx$ $0.1224)$. This figure illustrates two facts: (i) the successive PN approximants to $\hat{\delta}_{22}=1+c_{2} v_{y}^{2}+c_{3} v_{y}^{3}+c_{4} v_{y}^{4}+\cdots$ are suspiciously different from each other; and (ii) they introduce rather large fractional modifications of the LO phase $\delta_{22}^{\mathrm{LO}}(y) \equiv(7 / 3) y^{3 / 2}$ when $v_{y} \gtrsim 0.3$ (which is reached during the late plunge). This suggests a nonrobust behavior of the Taylor approximants in the highvelocity regime. In addition we have found that using $\delta_{22}^{\text {Taylor }}(y)$ in the generation of the EOB waveform generates pathological features in the waveform phase in the very late plunge phase, compromising the accuracy of the phasing in a crucial region. To overcome this difficulty, we replace $\hat{\delta}_{22}^{\text {Taylor }}\left(v_{y}\right)$ with its $(2,2)$ Padé approximant, i.e. we take $P_{2}^{2}\left[\hat{\delta}_{22}\left(v_{\Omega}\right)\right]$. Finally, we use in defining the factorized $\mathrm{EOB}$ waveform the following resummed version of the $\delta_{22}(y)$ phase:

$$
\delta_{22}(y) \equiv \delta_{22}^{\mathrm{LO}}(y) P_{2}^{2}\left[\hat{\delta}_{22}(\sqrt{y})\right]=\frac{7}{3} v_{y}^{3} \frac{p_{0}+p_{1} v_{y}+p_{2} v_{y}^{2}}{p_{0}+p_{1} v_{y}+p_{2}^{\prime} v_{y}^{2}},
$$

where $v_{y} \equiv y^{1 / 2}$. The explicit expressions of the $\nu$ dependent Padé coefficients $p_{0}(\nu), p_{1}(\nu), p_{2}(\nu), p_{2}^{\prime}(\nu)$ will be found in Appendix D. Note that this Padé representation degenerates as $\nu \rightarrow 0$, and yields $P_{2}^{2}\left[\hat{\delta}_{22}\left(v_{y}\right)\right] \rightarrow 1$; this occurs because the definition of this Padé approximant crucially depends on having a non-vanishing 3.5PN contribution. Figure 1 compares the Padé-resummed $\hat{\delta}_{22}\left(v_{y}\right)$ to its successive Taylor approximants. This figure suggests that the Padé approximant represents a reasonable "average" of the successive Taylor approximants.

We found that the (known) successive PN approximants to $\hat{\delta}_{21}^{\text {Taylor }}, \hat{\delta}_{33}^{\text {Taylor }}$ and $\hat{\delta}_{31}^{\text {Taylor }}$, exhibited a rather nonrobust behavior similar to that of $\hat{\delta}_{22}^{\text {Taylor }}$. We therefore decided to Padé resum them, using now $(1,2)$ Padé approximants, in view of the available PN knowledge. For the other residual phase corrections, $\delta_{32}, \delta_{4 m}$ with $m=1, \ldots, 4$ and $\delta_{55}$, there is too little PN information to try a resummation, so that we keep them in their unresummed Taylor-expanded form. See Appendix D for details.

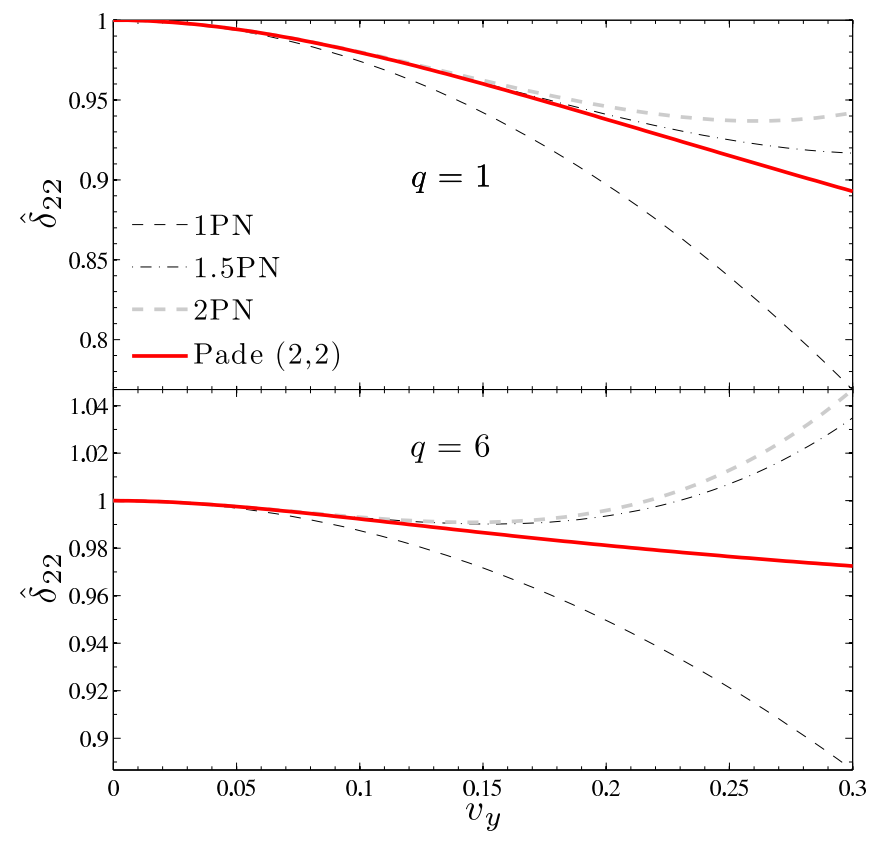

FIG. 1. (color online) Comparing the Taylor-expanded $\hat{\delta}_{22}$ with its $(2,2)$ Padé approximant for two mass ratios.

\section{Further factorized corrections to the waveform: $\rho_{\ell m}\left(v_{\varphi}^{2}\right)$ and $\hat{h}_{\ell m}^{\mathrm{NQC}}$.}

Let us first emphasize that, as in our previous work [22], we shall use as argument in the modulus correction $\rho_{\ell m}$ (to replace the generic variable $x$ used in [5]) the quantity $x_{\varphi}=v_{\varphi}^{2}=\left(r_{\omega} \Omega\right)^{2}$ defined above. By contrast, Ref. [28] uses $x=(M \Omega)^{2 / 3}$ as argument in the $\rho_{\ell m}$ 's. The $\rho_{\ell m}$ 's that enter Eq. (16) are taken at the complete $3^{+2} \mathrm{PN}$ approximation (as done in previous work $[26,27,31,42,52]$ ), i.e., by completing the $3 \mathrm{PN}$ accurate, $\nu$-dependent results of Ref. [5] by the $\nu=0$, $5 \mathrm{PN}$-accurate, terms obtained ${ }^{6}$ by Fujita and Iyer [51]. Note that in doing so we are taking into account more test-mass terms in the $\rho_{\ell m}$ 's than was done in Ref. [28], which was stopping one PN order earlier for $\rho_{33}, \rho_{31}$, $\rho_{4 m}$, and two PN orders earlier for $\rho_{5 m}, \rho_{6 m}$ and $\rho_{7 m}$. For completeness we list in Appendix D the explicit expressions of the $\rho_{\ell m}$ 's that we use. As we said, one must replace the generic variable $x$ used in these expressions by $x_{\varphi}=v_{\varphi}^{2}=\left(r_{\omega} \Omega\right)^{2}$.

Let us now discuss the structure of the final, NQC factor $\hat{h}_{\ell m}^{\mathrm{NQC}}$ in the factorized waveform, Eq. (16), as well as the procedure we shall use to determine (from NR data) the values of the coefficients $a_{i}^{\ell m}$ and $b_{i}^{\ell m}$ entering this NQC correction factor $\hat{h}_{\ell m}^{\mathrm{NQC}}$. We shall adopt here a more elaborate NQC factor $\hat{h}_{\ell m}^{\mathrm{NQC}}$ than what was considered in previous EOB literature. In particular, for each

\footnotetext{
${ }^{6}$ In successive steps, this computation has been recently pushed to the remarkable $22 \mathrm{PN}$ order by Fujita $[53,54]$.
} 
multipole $(\ell, m)$ this NQC factor depends on 6 real parameters, 3 for the amplitude, $a_{i}^{\ell m}, i=1, \ldots, 3$, and 3 for the phase $b_{i}^{\ell m}, i=1, \ldots, 3$ and reads

$$
\hat{h}_{\ell m}^{\mathrm{NQC}}=\left(1+\sum_{j=1}^{3} a_{j}^{\ell m} n_{j}\right) \exp \left(i \sum_{j=1}^{3} b_{j}^{\ell m} n_{j+3}\right),
$$

where the $n_{i}$ 's factors are chosen here to be

$$
\begin{aligned}
& n_{1}=\left(\frac{p_{r_{*}}}{r \Omega}\right)^{2} \\
& n_{2}=\frac{(\ddot{r})^{(0)}}{r \Omega^{2}}, \\
& n_{3}=n_{1} p_{r_{*}}^{2}, \\
& n_{4}=\frac{p_{r_{*}}}{r \Omega}, \\
& n_{5}=n_{4}(r \Omega)^{2}, \\
& n_{6}=n_{5} p_{r_{*}}^{2} .
\end{aligned}
$$

Here, the superscript (0) on the right-hand side of the definition of $n_{2}$ means that the second time derivative of $r$ is evaluated along the conservative dynamics (i.e. neglecting the contributions proportional to $\mathcal{F}$, see Appendix A for a discussion).

One should keep in mind that the EOB (dynamical) time $t^{\mathrm{EOB}}$ differs from the NR (retarded) time $t^{\mathrm{NR}}$ by an apriori unknown constant shift: $t^{\mathrm{EOB}}=t^{\mathrm{NR}}+\tau$. Determining $\tau$ is equivalent to the problem of aligning the NR and EOB waveforms. Physically, determining $\tau$ is equivalent to identifying one specific feature in the EOB waveform to a corresponding specific feature in the NR one. This choice has been different in various EOB-related works. From the beginning, i.e. [2], it was emphasized that a good marker on the EOB time axis of the "moment of merger" was the time $t_{\Omega \text { peak }}^{\mathrm{EOB}}$ where the EOB orbital frequency reaches its maximum. The issue is then to select the corresponding moment on the NR time axis. In all early EOB studies, it was assumed that the NR correspondent of $t_{\Omega \text { peak }}^{\mathrm{EOB}}$ is $t_{A_{22} \text { peak }}^{\mathrm{NR}}$, i.e. the $\mathrm{NR}$ instant when the $\ell=m=2$ amplitude reaches its maximum. However, several recent EOB-related works [29, 55, 56] gave evidence that, in the test-mass limit, the two instants $t_{\Omega \text { peak }}^{\mathrm{EOB}}$ and $t_{A_{22} \text { peak }}^{\mathrm{NR}}$ do not exactly correspond to each other.

In this work, we shall define the correspondence between $t^{\mathrm{EOB}}$ and $t^{\mathrm{NR}}$ by requiring that the correspondent on the NR time axis of the EOB instant $t_{\Omega \text { peak }}^{\mathrm{EOB}}$ is a specific time $t_{\text {extr }}^{\mathrm{NR}}$ which will be defined in Eq. (55) below. In addition, we shall use this time $t_{\Omega \text { peak }}^{\mathrm{EOB}} \leftrightarrow t_{\text {extr }}^{\mathrm{NR}}$ both as NQC determination point and as QNM attachment one ${ }^{7}$.

\footnotetext{
7 Note that this choice differs from the one used in Refs. [29, 56]. In these references the NQC and QNM EOB instant is chosen to be earlier than $t_{\Omega \text { peak }}^{\mathrm{EOB}}$ and to correspond to the NR instant $t_{A_{22} \text { peak }}^{\mathrm{NR}}$.
}

More precisely, for each multipole, the 6 parameters $a_{i}^{\ell m}$ and $b_{i}^{\ell m}$ entering Eq. (16) are determined from NR data by imposing that the EOB waveform $h_{\ell m}^{\mathrm{EOB}}\left(t^{\mathrm{EOB}}\right)$ (which is a function of the EOB dynamical time $t^{\mathrm{EOB}}$ ) "osculates" the NR waveform $h_{\ell m}^{\mathrm{NR}}\left(t^{\mathrm{NR}}\right)$ (which is a function of the NR retarded time $t^{\mathrm{NR}}$ ) around the NQCdetermination point $t_{\Omega \text { peak }}^{\mathrm{EOB}} \leftrightarrow t_{\text {extr. }}^{\mathrm{NR}}$

Note again that in this work we shall use as NQCdetermination point on the EOB time axis the EOB dynamical time $t_{\Omega \text { peak }}^{\mathrm{EOB}}$ when the $\mathrm{EOB}$ orbital frequency $\Omega\left(t^{\mathrm{EOB}}\right)$ reaches its (first) maximum ${ }^{8}$. The degree of osculation between the EOB and NR waveforms is defined by separately imposing a $C^{2}$ contact between the amplitudes, $A_{\ell m}$, and the frequencies, $\omega_{\ell m}$, of the two waveforms at the NQC-determination point $t_{\Omega \text { peak }}^{\mathrm{EOB}} \leftrightarrow t_{\text {extr. }}^{\mathrm{NR}}$. We do not constrain the relative phase of the EOB and NR waveforms. Explicitly, we impose the following six conditions

$$
\begin{aligned}
& A_{\ell m}^{\mathrm{EOB}}\left(t_{\Omega \text { peak }}^{\mathrm{EOB}}\right)=A_{\ell m}^{\mathrm{NR}}\left(t_{\text {extr }}^{\mathrm{NR}}\right), \\
& \dot{A}_{\ell m}^{\mathrm{EOB}}\left(t_{\Omega \text { peak }}^{\mathrm{EOB}}\right)=\dot{A}_{\ell m}^{\mathrm{NR}}\left(t_{\text {extr }}^{\mathrm{NR}}\right), \\
& \ddot{A}_{\ell m}^{\mathrm{EOB}}\left(t_{\Omega \text { peak }}^{\mathrm{EOB}}\right)=\ddot{A}_{\ell m}^{\mathrm{NR}}\left(t_{\text {extr }}^{\mathrm{NR}}\right), \\
& \omega_{\ell m}^{\mathrm{EOB}}\left(t_{\Omega \text { peak }}^{\mathrm{EOB}}\right)=\omega_{\ell m}^{\mathrm{NR}}\left(t_{\text {extr }}^{\mathrm{NR}}\right), \\
& \dot{\omega}_{\ell m}^{\mathrm{EOB}}\left(t_{\Omega \text { peak }}^{\mathrm{EOB}}\right)=\dot{\omega}_{\ell m}^{\mathrm{NR}}\left(t_{\text {extr }}^{\mathrm{NR}}\right), \\
& \ddot{\omega}_{\ell m}^{\mathrm{EOB}}\left(t_{\Omega \text { peak }}^{\mathrm{EOB}}\right)=\ddot{\omega}_{\ell m}^{\mathrm{NR}}\left(t_{\text {extr }}^{\mathrm{NR}}\right),
\end{aligned}
$$

which yield two separate $3 \times 3$ linear systems to be solved to obtain the $a_{i}^{\ell m}$ 's, and, separately, the $b_{i}^{\ell m}$ 's.

Note that the values of the $a_{j}^{\ell m}$ 's affect the modulus of the inspiral-plus-plunge waveform, which then affects the computation of the radiation reaction force (through the angular momentum flux, see below). In turn, this modifies the EOB dynamics itself, and, consequently, the determination of the $\left(a_{j}^{\ell m}, b_{j}^{\ell m}\right)$ 's. This means that one must bootstrap, by iteration, the determination of the $\left(a_{j}^{\ell m}, b_{j}^{\ell m}\right)$ 's until convergence (say at the third decimal digit) is reached. This typically requires three iterations. In previous work only the dominant $(2,2)$ NQC correction was included in the radiation reaction (though they were all taken into account when finally comparing EOB and NR waveforms). Here we shall follow the same simplifying prescription, though we have explored the effect of including also the subdominant $(2,1)$ and $(3,3)$ NQC corrections to the flux. We found that their effect amounts only to a small change in the NR determination of the "good values" of $a_{6}^{c}$ (see Appendix C).

\footnotetext{
8 This EOB time was often referred to, in previous works, as the "effective EOB light-ring crossing time", because, in the test-mass limit, it does correspond to the dynamical time when $R\left(t^{\mathrm{EOB}}\right)=3 M$, and, in the comparable-mass case, it is very close to the time when $R\left(t^{\mathrm{EOB}}\right)$ crosses the formal EOB analog of the light ring. Here, to avoid confusion, we shall call it the $\Omega$-peak time, and denote it as $t_{\Omega \text { peak }}^{\mathrm{EOB}}$.
} 
Summarizing: Our EOB waveform is given by Eq. (16) and employs the resummation of residual phases $\delta_{\ell m}$ as in Eq. (26). The NQC correction is defined by Eqs. (27)-(28) with constants determined from NR data by Eqs. (29).

\section{EOB waveform during merger and ringdown}

One of the specificities of the EOB formalism is to construct a complete waveform, covering the full process from early inspiral to ringdown, passing through late inspiral, plunge, and merger. This is done by attaching a sum of quasi-normal modes (QNM) to the end of the plunge waveform. The procedure for doing so has improved over the years $[2,8,45]$. Here, we use a new way of extending the inspiral-plus-plunge waveform to describe the merger-plus-ringdown subsequent signal, which fits with the NQC-determination procedure we have explained above. Our new procedure for, simultaneously, determining NQC corrections, and attaching QNM's, is motivated by the findings of Bernuzzi, Nagar and Zenginoglu [55] in the extreme mass ratio limit $(\nu \ll 1)$. We shall discuss the rationale for this procedure in the next Section.

The merger-plus-ringdown signal is described, for each multipole $\ell m$, by a sum of $N$ QNM signals of a final Kerr black hole (of mass $M_{f}$ and spin parameter $a_{f}$ ), say

$$
\left(\frac{R c^{2}}{G M}\right) h_{\ell m}^{\text {ringdown }}(t)=\sum_{n=0}^{N-1} C_{n}^{\ell m} e^{-\sigma_{n}^{+, \ell m}\left(t-t_{\Omega \text { peak }}^{\mathrm{EOB}}\right)},
$$

where $\sigma_{n}^{+, \ell m}=\alpha_{n}^{\ell m}+i \omega_{n}^{\ell m}$ is the complex frequency of the $n$th QNM of multipolarity $\ell m$ and $C_{n}^{\ell m}$ are complex constants.

In this work, we use $N=5$ positive frequency $\left(\omega_{n}^{\ell m}>\right.$ $0)$ QNM's. These complex frequencies are functions of the mass $M_{f}$ and spin parameter $a_{f}$ of the final hole [57]. For $M_{f}$ and $a_{f}$ we adopt the fit to the numerical results given in Eqs. (29) of [28],

$$
\begin{array}{r}
\frac{M_{f}}{M}=1+\left(\sqrt{\frac{8}{9}}-1\right) \nu-0.4333 \nu^{2}-0.4392 \nu^{3}, \\
\frac{a_{f}}{M}=\sqrt{12} \nu-3.871 \nu^{2}+4.028 \nu^{3} .
\end{array}
$$

The procedure we shall use here for matching the ringdown signal (30) to the inspiral-plus-plunge signal (16) is similar to the ones used in previous EOB work [55] though it differs in a significant way from the one used in [28]. Namely, contrary to the latter reference, the attachment (along the EOB dynamical time axis $t^{\mathrm{EOB}}$ ) of the QNM signal (30) to the NQC-corrected inspiral-plusplunge signal (16) is done, for each multipole $\ell m$, at the time

$$
t_{\ell m \mathrm{QNMattachment}}^{\mathrm{EOB}}=t_{\ell m \text { matching }}^{\mathrm{EOB}}=t_{\Omega \text { peak }}^{\mathrm{EOB}},
$$

where we recall that $t_{\Omega \text { peak }}^{\mathrm{EOB}}$ denotes the EOB dynamical time where the EOB orbital frequency reaches its (first) maximum. Note in particular that $t_{\Omega \text { peak }}^{\mathrm{EOB}}$ does not depend on the considered multipolarity $\ell m$, so that we are attaching the QNM's corresponding to all the different multipolarities at the same EOB dynamical time.

To complete the description of our QNM attachment procedure it remains to say that we determine, for each multipolarity $\ell m$, the values of the $N$ complex coefficients $C_{n}^{\ell m}$ by requiring that the (NQC-corrected) EOB inspiral-plus-plunge waveform $h_{\ell m}^{\text {insplunge }}\left(t^{\mathrm{EOB}}\right)$, Eq. (16), coincides with the QNM sum (30) at $N$ points, say $t_{1}, t_{2}, \cdots, t_{N}$, forming a regularly spaced "comb" on the $t^{\mathrm{EOB}}$ axis, centered on $t_{\Omega \text { peak }}^{\mathrm{EOB}}$. Such a "matching comb" is specified by choosing its total length, say

$$
\Delta^{\text {match }}=t_{N}-t_{1} .
$$

\section{Improved radiation reaction: Including horizon absorption and a radial component $\mathcal{F}_{r_{*}}$}

Let us now turn to our improved description of the radiation reaction force $\mathcal{F}$ entering the EOB dynamics. Note that we have included in the equations of motion (5) not only an azimuthal radiation reaction $\hat{\mathcal{F}}_{\varphi}$ (as in all previous EOB works), but also an explicit radial contribution $\hat{\mathcal{F}}_{r_{*}}$. We have improved the analytical description of both components of $\mathcal{F}$. Let us discuss them in turn.

The azimuthal component, $\mathcal{F}_{\varphi}$, of the radiation reaction force describes the loss of the orbital angular momentum $p_{\varphi}$ of the system during evolution. Indeed, Hamilton's equation for $p_{\varphi}$ reads

$$
\frac{d p_{\varphi}}{d t}=\hat{\mathcal{F}}_{\varphi}
$$

where $\hat{\mathcal{F}}_{\varphi}=\mathcal{F}_{\varphi} / \nu$.

Following a standard EOB practice (since Ref. [2]), we require that the loss of orbital angular momentum be balanced by the instantaneous flux of angular momentum leaving the orbital system. In previous EOB work, one took into account only the flux of angular momentum in the form of GWs at infinity. However, there is also a flux of angular momentum which is drained out of the two-point mass orbital system by penetrating within the two horizons of the moving black holes. [The latter flux is transformed from the orbital form measured by $p_{\varphi}$ to some intrinsic spin-angular momentum of the holes; from the point of view of the orbital $p_{\varphi}$ this represents a loss that must be accounted for by an additional contribution to $\mathcal{F}_{\varphi}$.] We shall include here such an additional horizon-absorption flux by using the recent work of Nagar and Akcay [41]. The corresponding effect is rather small and, in a PN sense, starts only at the $4 \mathrm{PN}$ level [58, 59]. Reference [59], using a leading-order (Newtonian) approximation both to the phase evolution and to the horizon flux had estimated that, in the nonspinning 
case (that we consider here), the inclusion of the horizon flux entails an additional dephasing at $R \approx 6 M$ smaller than $0.01 \mathrm{rad}$ for mass ratios $1 \leq q \leq 4$. On the other hand, recently Bernuzzi, Nagar and Zenginoglu [42], using an EOB description of the phase evolution together with an improved estimate of the horizon flux (resumming higher effects), have found significantly larger dephasings (accumulated over the last 20-30 orbits) than those estimated in [59]. Within the EOB model that we use here we confirmed the findings of Ref. [42]. For instance taking the most relevant case $q=6$ with initial separation $r_{0}=15$ (corresponding to $\sim 27$ orbits up to merger, see Table II below) the effect of horizon absorption entails a dephasing $\Delta^{H} \phi \equiv \phi^{H+\mathscr{I}}-\phi^{\mathscr{I}} \sim 0.12 \mathrm{rad}$ at $t_{\Omega \text { peak }}^{\mathrm{EOB}}$, that increases up to $0.18 \mathrm{rad}$ during ringdown ${ }^{9}$. Such dephasings are quite significant for the EOB/NR comparison that we shall perform below. This is why we decided to include the horizon contribution to the angular momentum flux.

It is convenient to decompose $\mathcal{F}_{\varphi}$ as the product of the usual quadrupolar GW flux (expressed in terms of $r_{\omega}$ and of the orbital frequency $\Omega=d \varphi / d t$ ) and of a supplementary dimensionless correction factor (of the $1+$ $O(x)$-type) :

$$
\hat{\mathcal{F}}_{\varphi}=-\frac{32}{5} \nu r_{\omega}^{4} \Omega^{5} \hat{f}\left(v_{\varphi}^{2} ; \nu\right) .
$$

Here the function $\hat{f}(x ; \nu)=1+O(x)$ (taken with the argument $x=v_{\varphi}^{2}$ ) is the reduced flux function. It can be defined, for a circularized binary, as the ratio between the total energy flux (including the horizon flux) and the $\ell=m=2$ asymptotic energy flux. In our case this function is given by the sum of an asymptotic (labeled by $\mathscr{I}$ ) and a horizon (labelled by $H$ ) contribution, and can be further written as

$$
\hat{f}(x ; \nu)=\hat{f}^{\mathscr{I}}(x ; \nu)+\left(1-4 \nu+2 \nu^{2}\right) x^{4} \hat{f}^{H}(x ; \nu),
$$

where each function $\hat{f}^{(\mathscr{I}, H)}(x ; \nu)$ is of the $1+O(x)$ type and is defined by dividing by the corresponding $\ell=m=$ 2 LO contribution, namely

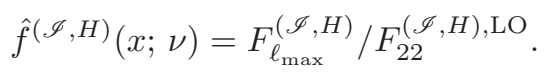

Here, $F_{\ell_{\max }}^{(\mathscr{I}, H)}$ is either the total asymptotic $(\mathscr{I})$ or horizon $(H)$ energy flux for circular orbits summed up to multipole $\ell=\ell_{\max }$, while $F_{22}^{\mathscr{I}, \mathrm{LO}}=(32 / 5) \nu^{2} x^{5}$ is the LO (or "Newtonian") quadrupolar (asymptotic) energy flux, and $F_{22}^{H, \mathrm{LO}}=(32 / 5) \nu^{2}\left(1-4 \nu+2 \nu^{2}\right) x^{9}=x^{4}(1-4 \nu+$ $\left.2 \nu^{2}\right) F_{22}^{\mathscr{J}, N}$ the LO quadrupolar horizon flux $[58,59]$. In the EOB model one uses suitably factorized expressions

\footnotetext{
9 Note that one has $\Delta^{H} \phi \sim 1.6 \times 10^{-4}$ at the initial separation $r_{0}=15$, which is negligible compared to the dephasing accumulated during the subsequent evolution.
}

TABLE I. Coefficients of our hybrid $1^{+3} \mathrm{PN}$-accurate $\rho_{\ell m}^{H}(x ; \nu)$ functions as given by Eq. (45).

\begin{tabular}{cccccc}
\hline \hline$\ell$ & $m$ & $c_{1}^{\ell m}$ & $c_{2}^{\ell m}$ & $c_{3}^{\ell m}$ & $c_{4}^{\ell m}$ \\
\hline 2 & 2 & $\frac{4-21 \nu+27 \nu^{2}-8 \nu^{3}}{4\left(1-4 \nu+2 \nu^{2}\right)}$ & 4.78752 & 26.760136 & 43.861478 \\
2 & 1 & 0.58121 & 1.01059 & 7.955729 & 1.650228 \\
\hline \hline
\end{tabular}

for the multipolar fluxes $F_{\ell m}^{(\mathscr{I}, H)}$ to resum and improve them with respect to standard $\mathrm{PN}$-expanded expressions in the strong-field, fast-velocity regime. In the case of the multipolar asymptotic flux $F_{\ell m}^{\mathscr{I}}$, this factorized flux is simply defined (as first proposed in [22]) by squaring the corresponding factorized multipolar waveform of [5], recalled above. An analogous procedure for the multipolar horizon fluxes, $F_{\ell m}^{H}$ was introduced in Ref. [41] and compared with Regge-Wheeler-Zerilli numerically computed horizon fluxes in Ref. [42]. [Here, we are considering nonspinning binaries.]

The horizon and asymptotic energy fluxes along circular orbits are then written as multipolar sums, say

$$
F^{\left[(\mathscr{I}, H), \ell_{\max }\right]}(x ; \nu)=\sum_{\ell=2}^{\ell_{\max }} \sum_{m=1}^{\ell} F_{\ell m}^{(\mathscr{I}, H, \epsilon)}(x ; \nu),
$$

where $F_{\ell m}^{(\mathscr{I}, H, \epsilon)}=F_{\ell|m|}^{(\mathscr{I}, H, \epsilon)}$ sums the two equal contributions corresponding to $+m$ and $-m(m \neq 0$ as the $m=0$ contributions vanish for circular orbits).

Inserting in the (circular) asymptotic multipolar flux contribution,

$$
F_{\ell m}^{(\mathscr{I}, \epsilon)}=\frac{1}{8 \pi}(m \Omega)^{2}\left|R h_{\ell m}^{(\epsilon)}\right|^{2},
$$

the factorized waveform (16) yields

$F_{\ell m}^{\mathscr{I}, \epsilon}=F_{\ell m}^{(N, \epsilon)}\left(\hat{S}_{\text {eff }}^{(\epsilon)}\right)^{2}\left|T_{\ell m}(y)\right|^{2}\left(\rho_{\ell m}(x ; \nu)\right)^{2 \ell} \hat{F}_{\ell m}^{(\mathscr{I}, \epsilon) \mathrm{NQC}}$,

where $F_{\ell m}^{(N, \epsilon)}$ is defined by inserting the Newtonian-order waveform in (40), and where each subsequent factor is the squared modulus of a corresponding PN-correction factor entering (16); e.g., $\hat{F}_{\ell m}^{(\mathscr{I}, \epsilon) \mathrm{NQC}}=\left|\hat{h}_{\ell m}^{\mathrm{NQC}}\right|^{2}=$ $\left(1+\sum_{j=1}^{3} a_{j}^{\ell m} n_{j}\right)^{2}$. Let us mention that $\left|T_{\ell m}(y)\right|^{2}$ can be explicitly written in the simple form

$$
\left|T_{\ell m}(y)\right|^{2}=\frac{1}{(\ell !)^{2}} \frac{4 \pi \hat{\hat{k}}}{1-e^{-4 \pi \hat{\hat{k}}}} \prod_{s=1}^{\ell}\left(s^{2}+(2 \hat{\hat{k}})^{2}\right) .
$$

Similarly the horizon partial multipolar fluxes are written in factorized form [41]

$$
F_{\ell m}^{(H, \epsilon)}(x ; \nu)=F_{\ell m}^{\left(H_{\mathrm{LO}}, \epsilon\right)}(x ; \nu)\left[\hat{S}_{\mathrm{eff}}^{(\epsilon)}(x ; \nu)\left(\rho_{\ell m}^{H}(x ; \nu)\right)^{\ell}\right]^{2} .
$$


where $\rho_{\ell m}^{H}(x ; \nu)=1+O(x)$ are the residual amplitude corrections to the horizon waveform. Following Refs. [41, $42]$ we use a $1^{+3} \mathrm{PN}$ approximation for $\rho_{\ell m}^{H}(x ; \nu)$ and we include only the $\ell=2$ contribution in Eq. (43) (i.e., we fix $\ell_{\max }=2$ in Eq. (39)).

Finally, this means that the fractional horizon correction (before multiplication by the additional factor $\left.1-4 \nu+2 \nu^{2}\right)$ in Eq. (37) is of the form

$x^{4} \hat{f}^{H}(x ; \nu)=x^{4}\left[\hat{S}_{\mathrm{eff}}^{(0)}\left(\rho_{22}^{H}(x ; \nu)\right)^{2}\right]^{2}+x^{5}\left[\hat{S}_{\mathrm{eff}}^{(1)}\left(\rho_{21}^{H}(x ; \nu)\right)^{2}\right]$

where $\hat{S}_{\text {eff }}^{(0)}=\hat{H}_{\text {eff }}, \hat{S}_{\text {eff }}^{(1)}=p_{\varphi} /\left(r_{\omega} v_{\varphi}\right)$, and where we use $4 \mathrm{PN}$ accurate expressions for $\rho_{\ell m}^{H}(x ; \nu)$,

$$
\rho_{\ell m}^{H}(x ; \nu)=1+c_{1}^{\ell m} x+c_{2}^{\ell m} x^{2}+c_{3}^{\ell m} x^{3}+c_{4}^{\ell m} x^{4},
$$

with values for the needed $\ell=2$ coefficients $c_{i}^{\ell m}, i=$ $1, \ldots, 4$ listed in Table I.

Let us finally come to discussing the radial component $\mathcal{F}_{r_{*}}$ of the radiation reaction force. Such a contribution was generally neglected in previous EOB papers, or replaced (e.g. in $[10,28]$ ) by an expression which was not consistently derived. Recently, Bini and Damour [43] (building on previous work by Iyer and collaborators [6062]) have shown that consistency with the usual EOB definition of $\hat{\mathcal{F}}_{\varphi}$ (as being equal to minus the instantaneous flux of angular momentum) required a specific form for $\hat{\mathcal{F}}_{r_{*}}$ which differed from previously used expressions.

The result of Ref. [43] that we use here has the form

$$
\hat{\mathcal{F}}_{r_{*}}=-\frac{5}{3} \frac{p_{r_{*}}}{p_{\varphi}} \hat{\mathcal{F}}_{\varphi}\left(1+c_{1}(\nu) u+c_{2}(\nu) u^{2}\right)
$$

where the coefficients entering the $2 \mathrm{PN}$ correction read [43]

$$
\begin{aligned}
& c_{1}(\nu)=-\frac{227}{140} \nu+\frac{1957}{1680}, \\
& c_{2}(\nu)=\frac{753}{560} \nu^{2}+\frac{165703}{70560} \nu-\frac{25672541}{5080320} .
\end{aligned}
$$

\section{E. Post-post-circular initial data}

The construction of initial data for the EOB dynamics has been refined in a series of works $[2,9,10,20]$. Here we shall use the post-post-circular prescription, introduced in 2007 (see Sec. III B of [9]), and then used in all subsequent EOB-related works by our group [15, 20$22,24-26,31,42,52]$. This choice allows one to start the EOB dynamics (with negligible initial eccentricity) at a frequency that is compatible with the initial frequency of the NR waveforms we shall use here $\left(M \omega_{22} \approx\right.$ 0.0345 approximately corresponding to initial separation $R_{0} \approx 15 M$, see Table II below). Note that, by contrast, Pan et al. [28], who use the less accurate post-circular initial data of Ref. [10], start their EOB runs at an initial radius $R_{0} \gtrsim 50 M$ (corresponding to an initial GW frequency $\left.M \omega_{22} \leq 0.005\right)$ in order to get a good circularization of the dynamics at the frequency where numerical simulations start.

For completeness, let us review here the construction of post-post-circular initial data for a given relative initial separation $r_{0}$. We introduce a formal bookkeeping parameter $\varepsilon$ (to be set to 1 at the end) in front of the radiation reaction $\mathcal{F}_{\varphi}$ in the EOB equations of motion. The quasi-circular inspiralling solution of the EOB equa$\left.{ }^{2}\right]^{2}$ tions of motion can then be formally expanded in powers of $\varepsilon$ as

$$
\begin{aligned}
p_{\varphi}^{2} & =j_{0}^{2}(r)\left(1+\varepsilon^{2} k_{2}(r)+\mathcal{O}\left(\varepsilon^{4}\right)\right), \\
p_{r_{*}} & =\varepsilon \pi_{1}(r)+\mathcal{O}\left(\varepsilon^{3}\right) .
\end{aligned}
$$

Here, $j_{0}^{2}(r)$ is the usual circular approximation to the inspiralling squared angular momentum as explicitly given by

$$
j_{0}^{2}(r)=-\frac{A^{\prime}(u)}{\left[u^{2} A(u)\right]^{\prime}}
$$

where the prime means $d / d u$ (recall $u \equiv 1 / r$ ). The order $\varepsilon$-approximation to $p_{r_{*}}$, i.e. $\pi_{1}(r)$ ("post-circular") is then obtained by approximating the left-hand side (l.h.s) of Eq. (6c) by $d p_{\varphi} / d t \approx d j_{0}(r) / d t=\left(d j_{0}(r) / d r\right)(d r / d t)$. This determines $d r / d t$ and thereby a corresponding value of $p_{r_{*}}$ using Eq. (6b) (where we neglect the $p_{r_{*}}^{3}$ contribution). This leads to the following explicit expression for $\pi_{1}(r)$ :

$$
\varepsilon \pi_{1}(r)=\left[\nu \hat{H}_{\mathrm{EOB}} \hat{H}_{\mathrm{eff}}\left(\frac{B}{A}\right)^{1 / 2}\left(\frac{d j_{0}}{d r}\right)^{-1} \hat{\mathcal{F}}_{\varphi}\right]_{0},
$$

where the subscript 0 indicates that the r.h.s. is evaluated at the leading circular approximation $\varepsilon \rightarrow 0$. The post-post-circular approximation to $p_{\varphi}^{2}$ (term $\varepsilon^{2} k_{2}$ above) is then obtained by approximating the l.h.s. of Eq. (6d) by

$$
\frac{d p_{r_{*}}}{d t} \approx \varepsilon \frac{d \pi_{1}(r)}{d r} \frac{d r}{d t},
$$

where the radial derivative $d \pi_{1}(r) / d r$ is numerically computed. This transforms Eq. (6d) in a linear equation for $p_{\varphi}^{2}$, which leads to an explicit expression for the $r$ dependent correction $\varepsilon^{2} k_{2}(r)$ introduced above. In solving for $p_{\varphi}^{2}$ we keep, for additional accuracy, the contribution proportional to $p_{r_{*}}^{4} \simeq \varepsilon^{4} \pi_{1}^{4}(r)$.

Table II lists the post-post-circular data (as a function of $r_{0}$ ) obtained by this procedure, as we have used them in the present study. Note that these values mainly depend on the parameters entering the $A$ function, $\left(a_{5}^{c}, a_{6}^{c}\right)$, and depend almost negligibly on the values of the NQC parameters $a_{i}^{\ell m}$ entering $\mathcal{F}_{\varphi}$ that appears on the r.h.s. of Eq. (52). Actually, the values listed in Table II were computed by keeping only the $\left(a_{1}^{22}, a_{3}^{22}\right)$ NQC contributions. 
TABLE II. Post-post-circular initial data for EOB dynamics that we shall consider in this paper to assure negligible initial eccentricity. They are obtained with the choice $a_{5}^{c}=23.5$ and $a_{6}^{c}(\nu)=[-110.5+129(1-$ $4 \nu)]\left[1-1.5 \times 10^{-5} /(\nu-0.26)^{2}\right]^{1 / 2}$.

\begin{tabular}{cccccc}
\hline \hline$q$ & $\nu$ & $r_{0}$ & $p_{\varphi}$ & $p_{r}$ & $p_{r_{*}}$ \\
\hline 1 & 0.25 & 16 & 4.42467206 & -0.00101207 & -0.00088970 \\
2 & $0 . \overline{2}$ & 15 & 4.31684166 & -0.00113064 & -0.00098466 \\
3 & 0.1875 & 15 & 4.31889270 & -0.00096445 & -0.00083930 \\
4 & 0.1600 & 15 & 4.32052406 & -0.00083018 & -0.00072202 \\
6 & 0.1224 & 15 & 4.32276101 & -0.00064296 & -0.00055874 \\
\hline \hline
\end{tabular}

\section{F. Analytically unknown parameters, choices to be made, NR-completion of the EOB model}

Let us summarize the parameters entering the construction of our EOB model, emphasizing which parameters contain important dynamical information, which ones are already known with sufficient accuracy, which ones depend on reasonable choices we can make, and how NR data can be used to complete the EOB model by determining the various parameters.

At face value, the EOB model defined above depends on quite a few analytically unknown parameters, namely: $a_{5}^{c}(\nu), a_{6}^{c}(\nu)$, the $6 \mathrm{NQC}$ parameters $\left(a_{i}^{\ell m}, b_{i}^{\ell m}\right)$ for each waveform multipole, the values of the mass $M_{f}$ and spin $a_{f}$ of the final black hole, the number $N$ of QNM modes used in the ringdown signal, and the width $\Delta^{\text {match }}$ of the QNM matching comb.

Our attitude towards the use of NR data to complete the EOB model by determining these parameters is the following:

(i) As already said, we think (in view of previous EOB results $[22,28])$ that it is a reasonable choice to impose some a priori relation between $a_{5}^{c}(\nu)$ and $a_{6}^{c}(\nu)$, so as to look only for one free dynamical parameter. Here we shall fix $a_{5}^{c}(\nu)$ to the simple value $a_{5}^{c}(\nu)=23.5, \mathrm{Eq} .(13)$. This leaves only $a_{6}^{c}(\nu)$ as free parameter. We shall discuss below (see Section $\mathrm{V}$ ) how the nonperturbative information contained in NR phasing data can be used to determine the value of $a_{6}^{c}(\nu)$, in a way which is nearly decorrelated from the uncertainties in the determination of other parameters. Let us already indicate here a possible analytical fit to represent the, essentially linear in $\nu$, final result we shall get for this EOB parameter

$$
\begin{aligned}
a_{6}^{c}(\nu) & =(-110.5+129(1-4 \nu)) \\
& \times\left(1-\frac{0.000015}{(\nu-0.26)^{2}}\right)^{1 / 2}
\end{aligned}
$$

from Caltech-Cornell-CITA data.
We think that the NR determination of $a_{6}^{c}(\nu)$ leads to important information about the conservative dynamics of binary black holes (as we shall illustrate below);

(ii) Concerning the NQC parameters $\left(a_{i}^{\ell m}, b_{i}^{\ell m}\right)$, the procedure explained above reduces their determination from nonperturbative $\mathrm{NR}$ data to a single choice, namely that of the time $t_{\text {extr }}^{\mathrm{NR}}$ on the NR (retarded) time axis corresponding to the EOB time $t_{\Omega \text { peak }}^{\mathrm{EOB}}$ (which can be thought of as defining the "EOB merger time"). The choice of $t_{\text {extr }}^{\mathrm{NR}}$ on the NR time axis is not a matter of convention, but has (a priori) important physical consequences. It must be done by combining information coming both from comparable-mass NR simulations, and from extreme-mass-ratio ones. For reasons that shall be discussed below, we shall choose, for each mass ratio $\nu$, a specific value of $t_{\text {extr }}^{\mathrm{NR}}(\nu)$ given by

$$
\begin{aligned}
t_{\text {extr }}^{\mathrm{NR}}(\nu) & =t_{A_{22} \text { peak }}^{\mathrm{NR}}(\nu) \\
& +f(\nu)\left(t_{\dot{\omega}_{22} \text { peak }}^{\mathrm{NR}}(\nu)-t_{A_{22} \text { peak }}^{\mathrm{NR}}(\nu)\right)
\end{aligned}
$$

where

$$
f(\nu)=\frac{1}{6}(1+3(1-4 \nu))
$$

and where $t_{A_{22} \text { peak }}^{\mathrm{NR}}(\nu)$ is the NR time when the NR quadrupolar amplitude reaches its peak, and $t_{\dot{\omega}_{22} \text { peak }}^{\mathrm{NR}}(\nu)$ the NR time when the quadrupolar frequency has an inflection point. Here $f(\nu)$ varies between $f(0)=2 / 3$ and $f(1 / 4)=1 / 6$ as $\nu$ varies between 0 and $1 / 4 . t_{\text {extr }}^{\mathrm{NR}}(\nu)$ always lies on the right of (i.e. later than) the NR time $t_{A_{22} \text { peak }}^{\mathrm{NR}}(\nu)$. We shall extract nonperturbative information from NR data by computing from the various multipolar NR waveforms a certain number of derivatives of their amplitudes and frequencies at the extraction point $t_{\text {extr }}^{\mathrm{NR}}(\nu)$.

(iii) Building on previous work, we shall use the simple (NR-based) analytical fits (31) for the mass and spin of the final black hole. Note, however, that, in principle, the EOB model (when NR-completed by NQC corrections up to merger) does yield, by itself, predictions for $M_{f}$ and $a_{f}[2,63]$. This might be useful in cases (e.g. with large, precessing spins) where one does not have in hand accurate analytical fits for the characteristics of the final black hole.

(iv) We shall use here $N=5$ QNM's, and as explained below, we shall fix $\Delta^{\text {match }}=0.7 M$ for all multipoles. Note, that by contrast, Ref. [28] uses $N=8$ QNM's, introduces "pseudo QNM's", and employs much larger matching intervals, which also vary with $\ell m$. [E.g., the latter reference uses $\Delta^{22}=5 M$ and $\Delta^{33}=12 M$.] 
(v) Let us finally note that (contrary to [28]) we shall not introduce adjustable parameters in the waveforms, nor shall we introduce special modifications to improve the behavior of some subdominant multipoles.

\section{NUMERICAL RELATIVITY INFORMATION, AND $Q_{\omega}$ DIAGNOSTIC}

\section{A. Overview of numerical waveforms data}

The NR data we use here to complete the EOB waveform were obtained with the Spectral Einstein Code (SpEC) developed by the Caltech-Cornell-CITA collaboration [64-68]. Specifically, we used the waveforms recently published in Ref. [34], coming from simulations of nonspinning black hole binaries with mass ratios $q=m_{1} / m_{2}=(1,2,3,4,6)$. Before their publication, these data were already used in some EOB/NR and PN/NR comparisons [28, 33, 69]. We address the reader to Ref. [34] for all technical details about the numerical setup and estimates of the accuracy. Here we only recall that these are the longest published waveforms to date (together with the 33 orbits, equal-mass waveform of Ref. [69]), with a number of gravitational wave cycles up to merger (here conventionally defined as the maximum of the modulus of the quadrupolar metric waveform $\left.\left|h_{22}^{\mathrm{NR}}\right|\right)$ respectively $N_{\mathrm{GW}}=\{33,31,31,31,43\}$. We made use of two different types of waveform data: curvature, $\psi_{\ell m}^{4}$, and metric, $h_{\ell m}$, extrapolated to infinite extraction radius. Indeed, the metric waveform $h_{\ell m}$ was also directly extracted from the numerical spacetime using a Regge-Wheeler-Zerilli-based (RWZ) approach ${ }^{10}$, see Appendix of Ref. [23] for a discussion.

\section{B. Estimating the NR $Q_{\omega}(\omega)$ function for the curvature waveform}

In this subsection we shall explain how we extracted from NR data a useful, intrinsic measure of the NR phase evolution, namely the $Q_{\omega}(\omega)$ function. This function is a convenient version of the "intrinsic phase acceleration" function $\alpha(\omega)$ introduced in Ref. [9], which was defined such that $d \omega / d t=\alpha(\omega)$. This function is an intrinsic measure of the time-domain phase evolution in the sense that it is independent of the two shift ambiguities that affect any time-domain phase, $\phi(t)$ : an arbitrary phase shift $\phi \rightarrow \phi+c$, and an arbitrary time shift $t \rightarrow t+\tau$. The $Q_{\omega}(\omega)$ function is defined as

$$
Q_{\omega}(\omega) \equiv \frac{\omega^{2}}{\alpha(\omega)}=\frac{\omega^{2}}{\dot{\omega}}
$$

10 This type of RWZ approach was initiated by Abrahams and Price [70] and first implemented in the form of Ref. [23] in Refs. $[71,72]$.

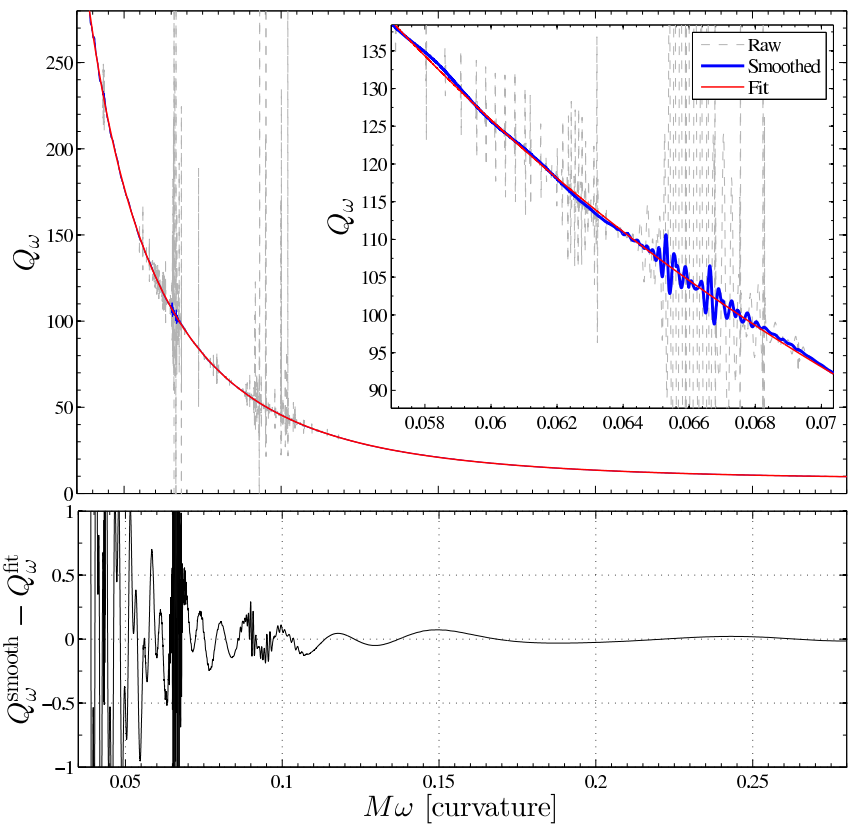

FIG. 2. (color online) Top panel: Raw NR, curvature waveform, data; smoothed data and fit. Bottom panel: difference between smoothed data and the fit.

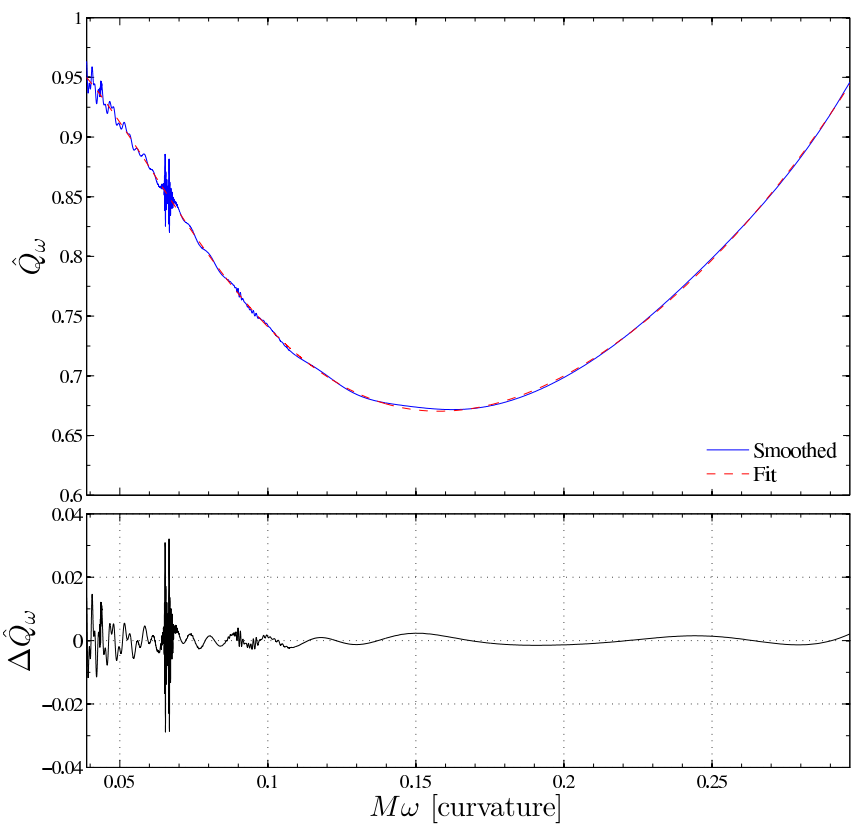

FIG. 3. (color online) Fitting the Newton-rescaled $\hat{Q}_{\omega}$, curvature waveform, function. The top panel contrasts the smoothed data with the outcome of the fit. The bottom panel shows their difference.

Note that this definition is equivalent to saying that the time-domain phase accumulated in the frequency interval $\left(\omega_{1}, \omega_{2}\right)$ is given by the integral

$$
\phi_{\left(\omega_{1}, \omega_{2}\right)}=\int_{\omega_{1}}^{\omega_{2}} Q_{\omega} d \ln \omega .
$$


TABLE III. Coefficients entering the fitting function for the Newton-rescaled, curvature-waveform, $\hat{Q}_{\omega}$, Eq. (64). In the second column we also report the frequency interval $M\left(\omega_{1}, \omega_{2}\right)$ on which the fit was performed.

\begin{tabular}{ccccccccccc}
\hline \hline$q$ & $M \omega_{1}$ & $M \omega_{2}$ & $n_{1}$ & $n_{2}$ & $n_{3}$ & $n_{4}$ & $n_{5}$ & $d_{1}$ & $d_{2}$ & $d_{3}$ \\
\hline 1 & 0.03877 & 0.29654 & -27.88757 & 256.94609 & -1053.85269 & 1926.40123 & -1274.57280 & -6.60927 & 47.87468 & -104.35366 \\
2 & 0.04133 & 0.29709 & 15.51565 & -372.20973 & 1725.17714 & -3145.40474 & 2105.30901 & -15.77371 & 90.80420 & -103.95952 \\
3 & 0.04476 & 0.29642 & 6.50413 & -243.11043 & 1108.15054 & -1913.96522 & 1193.58571 & -14.56312 & 79.22950 & -111.26577 \\
4 & 0.04819 & 0.29671 & 0.52391 & -172.68858 & 806.19352 & -1350.57200 & 797.20936 & -14.39733 & 78.72314 & -124.83300 \\
6 & 0.04280 & 0.29720 & 7.18353 & -247.53679 & 1096.28420 & -1833.39721 & 1090.77236 & -14.59256 & 75.97063 & -113.64331 \\
\hline \hline
\end{tabular}

The function $Q_{\omega}(\omega)$ has proven to be a very useful diagnostic of phase evolution in recent $\mathrm{EOB} / \mathrm{NR}$ comparisons of binary neutron stars [24, 25, 27]. Note that, in the definition, $\omega$ can be the frequency either of the curvature waveform, or of the metric one (thereby defining two different, though numerically close, functions). In general, one only considers the frequency of the dominant quadrupolar waveform, though one can also study the $Q_{\omega}(\omega)$ function of any $(\ell, m)$ multipole. Note also that we are here considering the phase acceleration of a time-domain phase. One can also usefully consider the frequency-domain counterpart of $Q_{\omega}(\omega)$, defined as $Q^{\mathrm{FD}}{ }_{\omega}(\omega) \equiv \omega^{2} d^{2} \psi(\omega) / d \omega^{2}$, where $\psi(\omega)$ denotes the phase of the Fourier-transformed waveform. In the stationary phase approximation, $Q_{\omega}^{\mathrm{FD}}(\omega)$ is simply equal to the time-domain $Q_{\omega}(\omega)$ (see, e.g., Eq. (17) in [31]).

Let us now discuss how to accurately estimate $Q_{\omega}(\omega)$ from the numerical data, in spite of the loss of accuracy associated to the fact that its definition (57) involves the computation of two derivatives of the phase $\phi(t)$. We consider the $\psi_{22}^{4}$ curvature waveform extrapolated to infinite extraction radius, decompose it in amplitude and phase with the convention

$$
\psi_{22}^{4}=\left|\psi_{22}^{4}\right| e^{-\mathrm{i} \phi_{22}}
$$

and consider as frequency in the definition (57) the curvature quadrupolar frequency: $\omega \equiv \dot{\phi}_{22}$.

It is somewhat of a challenge to get an accurate $Q_{\omega}$ out from numerical data. For example, in the case of binary neutron star waveforms, Refs. [24, 25, 27] argued that the successive straightforward differentiation (using finite-differencing, 4th-order stencils) of the numerical data is unable to get this information correctly, so that a suitable fitting of the GW phase was necessary to obtain something qualitatively and quantitatively correct. For general binary black hole simulations, due to the much higher resolution involved as well as due to the higher finite differencing operators used, direct differentiation could be more meaningful than in the binary neutron star case. This should be even more true for SpEC data, since they are expected to be particularly accurate.

Therefore, as a first step we directly computed $Q_{\omega}$ from the raw data simply by finite-differencing $\phi$ twice to get $\omega$ and $\dot{\omega}$, i.e., applying twice a 1st-derivative finitedifferencing operator with 4 th-order stencil. The result of this first step is shown, for $q=1$ data, as a dashed, light-gray line in Fig. 2 (see also the close up). The figure shows the presence of high frequency noise which prevents one from using this diagnostics as is for reliable quantitative estimates.

To improve on this, and get a quantitatively useful estimate of the $Q_{\omega}$ curve, we applied three more steps. First, in order to eliminate the high-frequency noise, we smoothed $\omega(t)$ with a Sgolay filter. Second, we computed the time derivative of the smoothed $\omega(t)$, and then smoothed again that derivative with a Sgolay filter. These two steps succeeded in strongly reducing the high-frequency noise in the curve (thick line in Fig. 2, blue online). However, there remained a low-frequency residual oscillation in the resulting $Q_{\omega}$ curve (evident in the inset of Fig. 2). We do not know the precise origin of this residual oscillation (it might either be related to some small residual eccentricity in the waveform or connected to the extrapolation procedure), but we think it is of spurious numerical origin and that it does not have any actual physical content (note that such an oscillation is not present in the $\mathrm{EOB} Q_{\omega}$ curve).

This led us to our third step: a fitting procedure of the $Q_{\omega}(\omega)$ function. To implement such a fitting procedure, it is convenient to first normalize the $Q_{\omega}$ curve with respect to its leading-order, Newtonian part,

$$
Q_{\omega}^{N}(\omega)=\frac{5}{3 \nu} 2^{-7 / 3} \omega^{-5 / 3}
$$

thereby factoring out the blowing up of $Q_{\omega}(\omega)$ at low frequency. The normalized function

$$
\hat{Q}_{\omega}(\omega)=Q_{\omega} / Q_{\omega}^{N}
$$

stays of order unity on the full frequency range (and $\hat{Q}_{\omega} \rightarrow 1$ for $\omega \rightarrow 0$ ) and is a better starting point for any fitting procedure (see Fig. 3 for $q=1$ ). Then we use as fitting template for $\hat{Q}_{\omega}$ a general analytical structure consistent with the structure of $\hat{Q}_{\omega}$ predicted by PN theory in the adiabatic approximation. More precisely, the 3.5PN-accurate expansion of $\hat{Q}_{\omega}$ is a Taylor expansion in half-integer powers of $x=(M \Omega)^{2 / 3}$ (modulo some logarithmic corrections) that reads

$\hat{Q}_{\omega}^{\mathrm{PN}}(x)=1+b_{2} x+b_{3} x^{3 / 2}+b_{4} x^{2}+b_{5} x^{5 / 2}+b_{6} x^{3}+b_{7} x^{7 / 2}$, 
where

$$
\begin{aligned}
b_{2} & =\frac{743}{336}+\frac{11}{4} \nu \\
b_{3} & =-4 \pi \\
b_{4} & =\frac{3058673}{1016064}+\frac{5429}{1008} \nu+\frac{617}{144} \nu^{2} \\
b_{5} & =\pi\left(-\frac{7729}{672}+\frac{13}{8} \nu\right) \\
b_{6} & =-\frac{10817850546611}{93884313600}+\frac{32}{3} \pi^{2} \\
& +\left(\frac{3147553127}{12192768}-\frac{451 \pi^{2}}{48}\right) \nu-\frac{15211}{6912} \nu^{2}+\frac{25565}{5184} \nu^{3} \\
+ & \frac{1712}{105}\left(\gamma_{E}+2 \log x+2 \log 2\right), \\
b_{7} & =-\pi\left(\frac{15419335}{1016064}+\frac{75703}{6048} \nu+\frac{14809}{3024} \nu^{2}\right)
\end{aligned}
$$

This motivated us to fit the smoothed version (coming out of the first two steps) of the numerically computed $\hat{Q}_{\omega}(\omega)$ with a Padé-type function of the form

$\hat{Q}_{\omega}^{\mathrm{fit}}\left(x_{\omega}\right)=\frac{1+n_{1} x_{\omega}+n_{2} x_{\omega}^{3 / 2}+n_{3} x_{\omega}^{2}+n_{4} x_{\omega}^{5 / 2}+n_{5} x_{\omega}^{3}}{1+d_{1} x_{\omega}+d_{2} x_{\omega}^{2}+d_{3} x_{\omega}^{3}}$,

where $x_{\omega} \equiv(M \omega / 2)^{2 / 3}$.

Let us now illustrate the result of performing this three-step evaluation of the numerical $Q_{\omega}(\omega)$ function. The top panel of Fig. 2 shows, for $q=1$, the three successive estimates of the numerical $\hat{Q}_{\omega}$ : the raw one (dashed line, featuring many large spikes), the smoothed one (solid line), and finally the fit obtained using the template (64). Note that all those curves are plotted versus $M \omega$. The bottom panel of the same figure shows the difference $\Delta \hat{Q}_{\omega}(\omega)=\hat{Q}_{\omega}^{\text {smoothed }}(\omega)-\hat{Q}_{\omega}^{\text {fit }}(\omega)$ between the smoothed data and the fit. Note that this difference is oscillating around 0 , which indicates that the fit has been effective in averaging away the low-frequency oscillation remaining after having smoothed the highfrequency noise. The procedure works in the same way for the other mass ratios, and for each one the difference $\Delta \hat{Q}_{\omega}(\omega)$ nicely oscillates around zero.

We list in in Table III, for all mass ratios, the fitting coefficients of the smoothed numerical $\hat{Q}_{\omega}$ to the template Eq. (64). Note that this list of coefficients provides a convenient way of condensing the information contained in the NR phasing during most of the inspiral and plunge (indeed, our fit worked well up to frequency $M \omega \simeq 0.3$, which is quite close to the merger). This packaging of the NR phasing information might be useful for many purposes, e.g., comparing various numerical simulations, computing the Fourier transform in the stationary-phase approximation, etc.

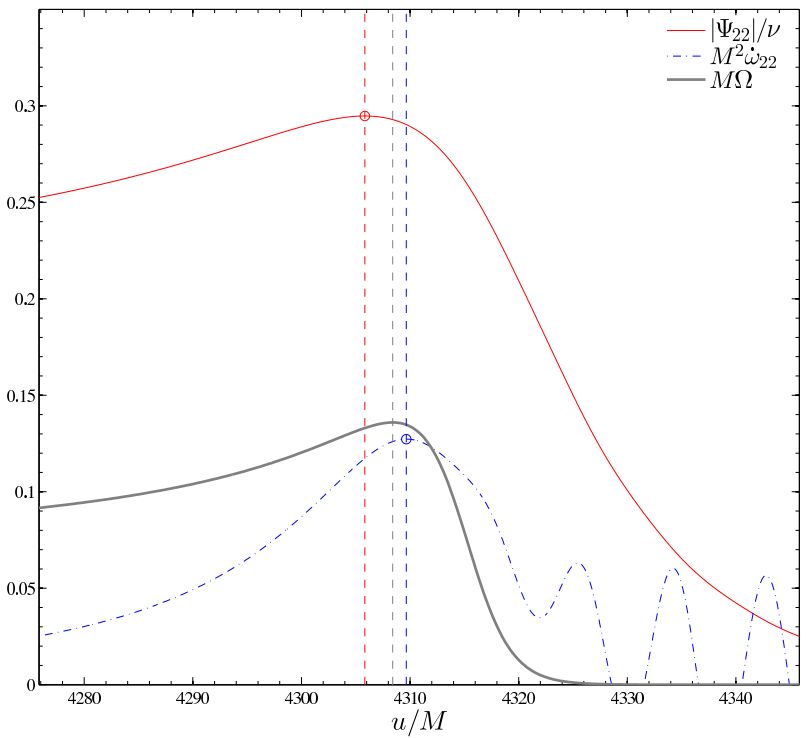

FIG. 4. (color online) Hierarchy of important points of the test-mass (Zerilli-normalized) quadrupolar metric waveform (divided by $\nu), \Psi_{22} / \nu \equiv(R / M) h_{22} /(\nu \sqrt{24})$ around the merger point. The orbital frequency $\Omega$ peaks at approximately $2 / 3$ of the time interval between the peak of the metric amplitude and the inflection point of the GW frequency, i.e. the first peak of $\dot{\omega}_{22}$

TABLE IV. Time intervals $t_{\dot{\omega}_{22} \text { peak }}^{\mathrm{NR}}-t_{A_{22} \text { peak }}^{\mathrm{NR}}$ for all numerical waveforms considered in this paper.

\begin{tabular}{ccc}
\hline \hline$q$ & $\nu$ & $t_{\dot{\omega}_{22} \text { peak }}^{\mathrm{NR}}-t_{A_{22} \text { peak }}^{\mathrm{NR}}$ \\
\hline 1 & 0.25 & 3.2493 \\
2 & $0 . \overline{2}$ & 3.4426 \\
3 & 0.1875 & 3.3261 \\
4 & 0.1600 & 3.5714 \\
6 & 0.1224 & 3.5681 \\
$\infty$ & 0 & 3.8158 \\
\hline \hline
\end{tabular}

\section{REVISITING TEST-MASS LIMIT RESULTS}

\section{A. The new information acquired from test-particle computations}

Before dealing with the Caltech-Cornell-CITA comparable-mass waveforms, we shall revisit in this Section the test-mass limit case $\nu \ll 1$ both to motivate our introduction of an NR extraction point $t_{\text {extr }}^{\mathrm{NR}}$ differing from the peak of the waveforms, and to test the performance of the basis of functions $n_{i}$ 's that we shall use in our NQC correction factor, (27).

State-of-the-art computations of multipolar RWZ waveforms for the plunge and merger of a test particle (of mass $\mu$ ), moving in a Schwarzschild background (of mass 

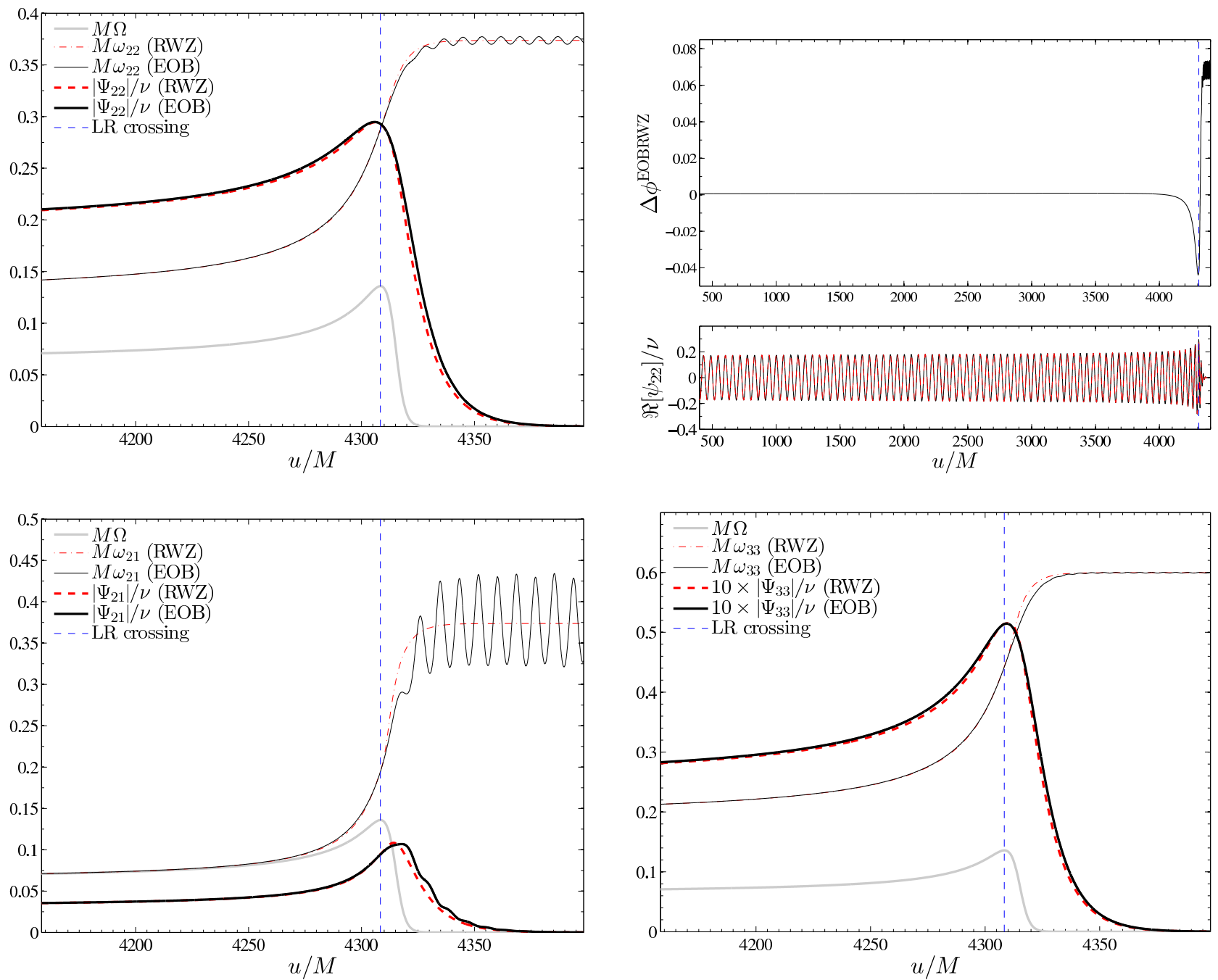

FIG. 5. (color online) Test-mass waveform: comparison between RWZ waveform extracted at $\mathscr{I}^{+}$and EOB waveform completed by the 6-parameter NQC correction factor to the waveform, Eq. (27). Top panels: $\ell=m=2$ multipole, modulus and frequency (left) and phasing (right). Bottom panels: $\ell=2$ and $m=1$ and $\ell=m=3$ frequency and modulus. The ringdown is modeled using 5 (positive-frequency only) QNMs.

$M)$, and submitted to a leading-order EOB resummed radiation-reaction force, have been presented in a recent series of works $[42,55,73,74]$. These works have used a recently developed method [75-77] allowing one to combine an accurate treatment of the particle motion in the strong field region, with the extraction of the waveforms directly at null infinity $(\mathscr{I})$. The findings of Ref. [55] that will be of direct interest for our present study are:

(i) The extraction of the waveforms at $\mathscr{I}$ allows one to relate the retarded time $t^{\mathrm{NR}}$ used as argument of the waveforms to the $\mathrm{EOB}$ time $t^{\mathrm{EOB}}$ used in the dynamics of the particle (namely, one has simply $\left.t^{\mathrm{NR}}=t^{\mathrm{EOB}}\right)$. This allows one to connect without ambiguity features in the waveform (such as, say, a peak in the modulus of $\left.h_{22}\left(t^{\mathrm{NR}}\right)\right)$ with features in the dynamics (such as, say, the location along the $t^{\mathrm{EOB}}$ axis of the maximum of the orbital fre- quency $\left.\Omega\left(t^{\mathrm{EOB}}\right)\right)$. Such a possibility is not available in comparable-mass NR simulations, because they do not track the light cones emitted by the center of mass of the binary system. In addition, even if they did, this would not allow one to relate the dynamical EOB time $t^{\mathrm{EOB}}$ to the waveform time $t^{\mathrm{NR}}$, because we would not know the exact relation between $t^{\mathrm{EOB}}$ and the NR coordinate time relevant for the NR dynamics.

(ii) Using the connection between the waveform time $t^{\mathrm{NR}}$ and the dynamical time $t^{\mathrm{EOB}}$ offered by (i), it was found that the waveform amplitude $A_{22}$ peaks approximately $\approx 2.56 \mathrm{M}$ earlier than the orbital frequency $\Omega$, i.e. $t_{\Omega \text { peak }}^{\mathrm{EOB}} \approx t_{A_{22} \text { peak }}^{\mathrm{NR}}+2.56 M$. This is new information which conflicts with the standard simplifying EOB assumption of a coincidence between the peaks of $A_{22}$ and of $\Omega$. The existence of 


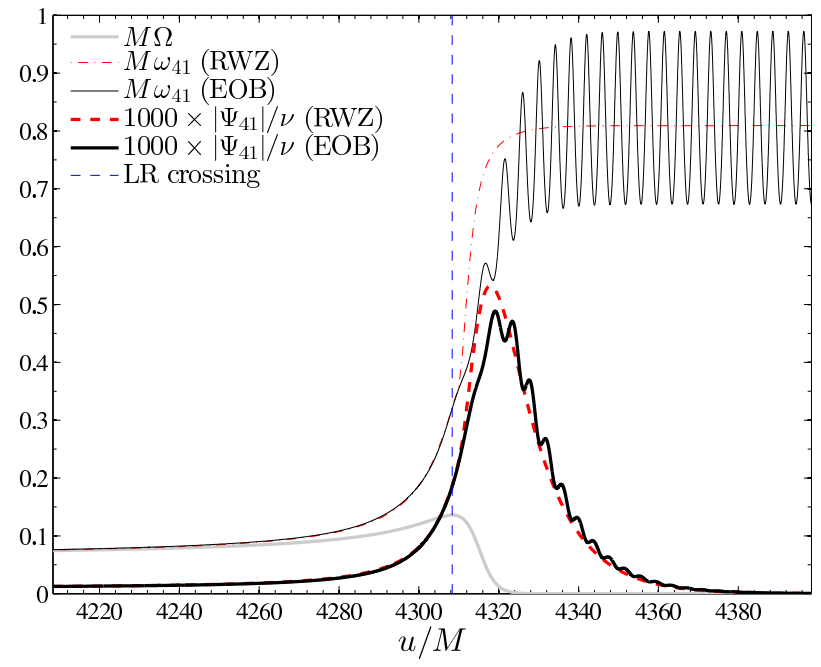

FIG. 6. (color online)Test-mass limit: comparison between $\ell=4, m=1 \mathrm{EOB}$ and RWZ modulus and frequency

a difference between $t_{\Omega \text { peak }}^{\mathrm{EOB}}$ and $t_{A_{22} \text { peak }}^{\mathrm{NR}}$ was later confirmed in Ref. [56] and extended to the case of a spinning central black hole.

(iii) Using this new information, Ref. [55] suggested to incorporate it in a new prescription for the determination of the EOB NQC correction factor based on extracting numerical data at the NR point $t_{\text {extr }}^{\mathrm{NR}}$ corresponding to $t_{\Omega \text { peak }}^{\mathrm{EOB}}$, rather $\operatorname{than}^{11}$ at $t_{A_{22} \text { peak }}^{\mathrm{NR}}$. They implemented such a prescription by imposing a $C^{1}$ contact at $t_{\text {extr }}^{\mathrm{NR}} \leftrightarrow t_{\Omega \text { peak }}^{\mathrm{EOB}}$ both (for the first time) between the modulus and the frequency of the waveform. They then showed that such a procedure produced NQC-corrected EOB waveforms which had an excellent agreement with the numerical RWZ waveforms up to merger.

The procedure we indicated in Eqs. (29a)-(29f) above is a generalization of this prescription to a $C^{2}$ contact requirement. We shall test below the increased accuracy brought by using such a $C^{2}$ contact requirement, involving six NQC-parameters, instead of the $C^{1}$ contact requirement used in Ref. [55], which involved only four NQC parameters. This test will also probe the new basis of NQC correction functions $n_{i}$ 's used in Eq. (27).

\section{B. Zooming on the structure of the test-mass waveform near merger}

Before doing the latter test, let us display the finding (ii) of Ref. [55] by investigating in detail the structure of

11 Note that, by contrast, Ref. [29] has chosen to keep, for $\ell=m=$ 2 the NR extraction point at $t_{A_{22} \text { peak }}^{\mathrm{NR}}$ and to map it to an EOB time earlier than $t_{\Omega \text { peak }}^{\mathrm{EOB}}$. the $\ell=m=2$ RWZ waveform around the peak of the modulus, with the idea that a similar structure might hold in the comparable mass case.

Figure 4 shows together (as functions of the waveform retarded time $u$, which can be identified with the EOB dynamical time): the waveform modulus $A_{22} / \nu$; the orbital frequency $\Omega$; and the derivative of the GW frequency $\dot{\omega}_{22}$. Here, $A_{22}$ is the modulus of the Zerilli-normalized quadrupolar metric testmass waveform, $\Psi_{22} \equiv(R / M) h_{22} / \sqrt{24}$. [For a general multipole the Zerilli normalized metric waveform is $\Psi_{\ell m} \equiv(R / M) h_{\ell m} / \sqrt{(\ell+2)(\ell+1)(\ell)(\ell-1)}$.] The figure clearly illustrates how the orbital frequency peaks at a time $t_{\Omega \text { peak }}^{\mathrm{EOB}}$ that is between the locations of the maxima of $A_{22}$ and $\dot{\omega}_{22}$, i.e. we have the relation $t_{A_{22} \text { peak }}^{\text {NR }}<$ $t_{\Omega \text { peak }}^{\mathrm{EOB}}<t_{\dot{\omega}_{22} \text { peak }}^{\mathrm{NR}}$. Quantitatively, given that we have $t_{\Omega \text { peak }}^{\mathrm{EOB}}-t_{A_{22} \text { peak }}^{\mathrm{NR}}=2.565388 M$ and $t_{\dot{\omega}_{22} \text { peak }}^{\mathrm{NR}}-t_{A_{22} \text { peak }}^{\mathrm{NR}}=$ $3.815784 M$ we have that

$$
\frac{t_{\Omega \text { peak }}^{\text {EOB }}-t_{A_{22} \text { peak }}^{\mathrm{NR}}}{t_{\dot{\omega}_{22} \text { peak }}^{\mathrm{NR}}-t_{A_{22} \text { peak }}^{\mathrm{NR}}}=\frac{2.565388}{3.815784}=0.6723096 \approx \frac{2}{3} .
$$

The comparable-mass NR simulations show that the ordering $t_{A_{22} \text { peak }}^{\mathrm{NR}}<t_{\dot{\omega}_{22} \text { peak }}^{\mathrm{NR}}$ remains true for all values of $\nu$ (for nonspinning binaries). By continuity, one then also expects that the EOB orbital frequency will continue to peak between these two points for any value of $\nu$. In other words, one expects that the correspondence between the EOB and NR time axes should be such that the EOB dynamical time $t_{\Omega \text { peak }}^{\mathrm{EOB}}(\nu)$ corresponds to an NR waveform time $t_{\text {extr }}^{\mathrm{NR}}(\nu)$ such that $t_{A_{22} \text { peak }}^{\mathrm{NR}}(\nu)<t_{\text {extr }}^{\mathrm{NR}}(\nu)<$ $t_{\dot{\omega}_{22} \text { peak }}^{\mathrm{NR}}(\nu)$ for any $\nu$. It is convenient to rewrite these inequalities as

$t_{\text {extr }}^{\mathrm{NR}}(\nu)-t_{A_{22} \text { peak }}^{\mathrm{NR}}(\nu)=f(\nu)\left(t_{\dot{\omega}_{22} \text { peak }}^{\mathrm{NR}}(\nu)-t_{A_{22} \text { peak }}^{\mathrm{NR}}(\nu)\right)$

where $f(\nu)$ is an unknown function satisfying the condition that $f(0)=2 / 3$, and expected to remain positive for any $\nu$. The intervals $t_{\dot{\omega}_{22} \text { peak }}^{\mathrm{NR}}-t_{A_{22} \text { peak }}^{\mathrm{NR}}$ as measured on the numerical waveforms are listed in Table IV. We shall discuss our choice for the function $f(\nu)$ in the following Section.

\section{Testing the improvements brought by requiring} a $C^{2}$ contact when using the NQC factor Eq. (27)

Reference [55] was able to build a rather satisfactory EOB waveform modulus and frequency up to merger for the $\ell=m=2$ mode (and in general for all $\ell=m$ modes) by using four NQC parameters (two for the amplitude and two for the phase). However, their results for the modulus were much less satisfactory for the other $(\ell \neq$ $m$ ) subdominant multipoles, such as the $\ell=2, m=1$ one. Let us show here how the use of the new NQC factor, Eq. (27) (which contains six NQC parameters, and uses different choices for the NQC functions $n_{3}$ and $n_{4}$ ) improves the closeness of the EOB waveform to the 
numerical (RWZ) one. To be consistent with Ref. [55], the EOB dynamics used for this comparison is slightly different from the one we discussed above. Namely: (i) we set to zero $\mathcal{F}_{\varphi}^{H}$, i.e. the horizon-absorption part of the radiation reaction; (ii) we also set $\mathcal{F}_{r_{*}}=0$; (iii) in addition, the residual phase corrections $\delta_{\ell m}$ for $\nu=0$ are considered in their Taylor-expanded form and all terms (up to 4.5PN accuracy) are included (see Appendix D).

The improved EOB waveform obtained by using the new six-parameter NQC factor is illustrated in Fig. 5. The top panels refer to the $\ell=m=2$ mode: frequency and modulus (left) and phasing (right). The bottom panels compare EOB and RWZ frequency and modulus for $\ell=2, m=1$ (left) and $\ell=m=3$ (right). For all waveforms the QNM matching comb has a total width $\Delta=0.7 M$ and we use five, positive frequency, QNMs. The restriction to positive frequency QNMs is the reason why one cannot reproduce the oscillations during ringdown in the $\ell=2, m=1$ mode. The improvement with respect to Fig. 3 of Ref. [55] is evident. Notably, the $\ell=2$ $m=1$ modulus comes out extremely well (modulo the absence of negative-frequency modes to model the ringdown). The $\ell=m=2$ phasing remains good also during merger and ringdown $-0.05<\Delta \phi^{\text {EOBRWZ }}<+0.05$ (while the QNM matching of Ref. [55] led to significantly larger dephasings during ringdown). Note on the top right panel of Fig. 5 the behavior of the phase difference: it dips just before merger down to $-0.04 \mathrm{rad}$, and then jumps up to $+0.06 \mathrm{rad}$ during ringdown. Such a behavior is a useful compromise for keeping, on average, a good phasing through inspiral, plunge, merger and ringdown.

Finally, to prove the robustness of the NQC determination procedure and the accuracy of the EOB waveform for higher multipoles, we show in Fig. 6 the $\ell=4, m=1$ frequency and modulus. The agreement between EOB and RWZ waveform is again very good, modulo the absence of negative modes in the ringdown modelization.

\section{COMPARABLE MASS CASE: $a_{6}^{c}(\nu), t_{\mathrm{extr}}^{\mathrm{NR}}(\nu)$, AND PHASING PERFORMANCE}

\section{A. Iterative procedure for determining $t_{\mathrm{extr}}^{\mathrm{NR}}(\nu)$ and $a_{6}^{c}(\nu)$ overview}

After having tested the performance of the NQC factor (27) in the test-mass limit, we now move to the comparable-mass case. Let us explain how we distilled crucial nonperturbative information out of the CaltechCornell-CITA waveform data. Our aim was to determine good values of the $5 \mathrm{PN}$ parameter $a_{6}^{c}(\nu)$, and of the NR time $t_{\mathrm{extr}}^{\mathrm{NR}}(\nu)$ corresponding to the EOB time $t_{\Omega \text { peak }}^{\mathrm{EOB}}$. We recall that $t_{\text {extr }}^{\mathrm{NR}}(\nu)$ is parametrized by a function $f(\nu)$, according to Eq. (66). Actually, the determinations of $a_{6}^{c}(\nu)$, and of $t_{\text {extr }}^{\mathrm{NR}}(\nu)$ are correlated, and must be done essentially simultaneously. From a practical point of view, we used an iterative, trial and error method.

First, for a given mass ratio $\nu$, and a given choice of
NR extraction time $t_{\text {extr }}^{\mathrm{NR}}$ (chosen around merger), we extract, from the behavior of the waveform in the immediate vicinity of the retarded time, $t_{\text {extr }}^{\mathrm{NR}}$, a collection of NR waveform quantities $\left(A_{\ell m}^{\mathrm{NR}}, \dot{A}_{\ell m}^{\mathrm{NR}}, \ddot{A}_{\ell m}^{\mathrm{NR}}, \omega_{\ell m}^{\mathrm{NR}}, \dot{\omega}_{\ell m}^{\mathrm{NR}}, \ddot{\omega}_{\ell m}^{\mathrm{NR}}\right)$. [As mentioned above, these quantities are then used, for any given value of $a_{6}^{c}(\nu)$, to determine the parameters $\left(a_{i}^{\ell m}, b_{i}^{\ell m}\right)$ entering the EOB NQC factor; i.e. the last factor in the pre-merger EOB waveform (16).] Second, we study how the phase difference $\Delta \phi^{\mathrm{EOBNR}}$ between the so determined NQC-corrected EOB waveform and the NR waveform evolves (either as a function of frequency, or of time) from the beginning of the simulation up to $t_{\text {extr }}^{\mathrm{NR}}$. The evolution of the phase difference $\Delta \phi^{\mathrm{EOBNR}}$ depends (after having chosen $t_{\mathrm{extr}}^{\mathrm{NR}}$, and having implemented the previous step) only on the 5PN ( $\nu$-dependent) parameter $a_{6}^{c}(\nu)$. We then search (for each $\nu$ ) whether there exist values of $a_{6}^{c}(\nu)$ which entail that $\Delta \phi^{\operatorname{EOBNR}}\left(a_{6}^{c}(\nu)\right)$ remains within the numerical uncertainty during the full simulation (up to $t_{\mathrm{extr}}^{\mathrm{NR}}$ ). If such a tuning of $a_{6}^{c}(\nu)$ does not seem to lead to a a satisfactorily small phase discrepancy during the whole evolution, we try another value of the NR extraction time and repeat the two steps above, until we end up with a better pair $\left(t_{\mathrm{extr}}^{\mathrm{NR}}, a_{6}^{c}(\nu)\right)$.

When completed (by iteration), the above two steps completely define an NR-completed EOB model up to merger. The EOB waveform is then extended through merger and ringdown by attaching QNMs at the end of the inspiral-plus-plunge waveform, i.e. at the EOB time $t_{\Omega \text { peak }}^{\mathrm{EOB}}$ (which corresponds to the NR time $t_{\text {extr }}^{\mathrm{NR}}$ ). This extension does not require the extraction of further NR information, but only requires to choose, by trial and error, reasonably good values of the number of QNM modes $N$, and of the total width of the matching comb $\Delta^{\text {match }}$ around $t_{\Omega \text { peak }}^{\mathrm{EOB}}$. As already said, we use $N=5$ and $\Delta^{\text {match }}=0.7 M$.

\section{B. Determining $t_{\mathrm{extr}}^{\mathrm{NR}}(\nu)$}

We started by applying this iterative procedure to the equal-mass case $q=1$ (i.e. $\nu=0.25$ ). After trial and error, we concluded that, for $q=1$, the coefficient $f(\nu)$ in Eq. (66) could be taken to have the value $f(0.25)=$ $1 / 6$. In other words, when $q=1, t_{\text {extr }}^{\mathrm{NR}}$ can be taken to be rather close to the peak of the $A_{22}$ modulus, as was indeed assumed in all previous EOB works. By contrast, when considering larger mass ratios, we found more and more advantageous to increase the value of $f(\nu)$, up to values of order of the test-mass value discussed above, $f(0)=2 / 3$, for large mass ratios. Then, as a simplifying choice, we decided to assume for the $\nu$ dependence of $f(\nu)$ a simple linear behavior between the two extreme values for $\nu=0$ and $\nu=0.25$, in the form

$$
f(\nu)=f(0.25)+(f(0)-f(0.25))(1-4 \nu) ;
$$


TABLE V. Next-to-quasi-circular $\left(a_{i}^{\ell m}, b_{i}^{\ell m}\right)$ coefficients needed to complete the EOB for the five mass ratios considered. They are obtained by imposing $C^{2}$ conditions to the waveform amplitude and frequency around the merger.

\begin{tabular}{|c|c|c|c|c|c|c|c|}
\hline$q$ & $\ell m$ & $a_{1}^{\ell m}$ & $a_{2}^{\ell m}$ & $a_{3}^{\ell m}$ & $b_{1}^{\ell m}$ & $b_{2}^{\ell m}$ & $b_{3}^{\ell m}$ \\
\hline \multirow[t]{2}{*}{1} & 22 & -0.0577 & 1.8127 & -0.1205 & 0.0794 & -0.9164 & -2.5890 \\
\hline & 32 & 0.0987 & 2.4076 & -0.4987 & 0.0490 & 1.0532 & -2.9188 \\
\hline \multirow[t]{4}{*}{2} & 21 & -0.0656 & 0.4871 & 0.2959 & 0.2544 & 0.9033 & 1.1975 \\
\hline & 22 & -0.0602 & 1.7571 & -0.0646 & 0.0963 & -0.8789 & -2.0165 \\
\hline & 32 & -0.0658 & 2.7289 & -0.2130 & 0.0864 & 1.2601 & -2.7701 \\
\hline & 33 & -0.0068 & 2.1915 & -0.1837 & 0.2300 & -1.2604 & -2.2847 \\
\hline \multirow[t]{4}{*}{3} & 21 & -0.0566 & 0.2988 & 0.3668 & 0.2636 & 0.8883 & 1.8284 \\
\hline & 22 & -0.0484 & 1.6672 & -0.0347 & 0.1161 & -0.7453 & -1.4052 \\
\hline & 32 & -0.1349 & 2.6377 & -0.0518 & 0.1747 & 1.5855 & -0.2960 \\
\hline & 33 & 0.0016 & 2.0213 & -0.0789 & 0.2560 & -1.1539 & -1.0416 \\
\hline \multirow[t]{4}{*}{4} & 21 & -0.0464 & 0.1260 & 0.4288 & 0.2772 & 1.0397 & 1.9334 \\
\hline & 22 & -0.0396 & 1.5639 & 0.0004 & 0.1342 & -0.5509 & -1.1731 \\
\hline & 32 & -0.1360 & 2.3134 & 0.0559 & 0.2795 & 1.9825 & -0.0350 \\
\hline & 33 & 0.0079 & 1.8294 & 0.0243 & 0.2807 & -0.9226 & -0.5157 \\
\hline \multirow[t]{4}{*}{$\bar{~} 6$} & 21 & -0.0323 & -0.0701 & 0.5183 & 0.2770 & 1.2750 & 2.0649 \\
\hline & 22 & -0.0229 & 1.4177 & 0.0397 & 0.1498 & -0.4375 & -0.9124 \\
\hline & 32 & -0.1114 & 1.7472 & 0.2487 & 0.3207 & 2.2001 & 1.5262 \\
\hline & 33 & 0.0296 & 1.5816 & 0.1347 & 0.3014 & -0.7664 & -0.1969 \\
\hline
\end{tabular}

which yields, when using $f(0.25)=1 / 6$ and $f(0)=2 / 3$, the explicit expression

$$
f(\nu)=\frac{2}{3}-2 \nu
$$

Having so chosen $t_{\text {extr }}^{\mathrm{NR}}(\nu)$, we measure, for each $(\ell, m)$, on the NR mutipolar waveform the vector $\left(A_{\ell m}^{\mathrm{NR}}, \dot{A}_{\ell m}^{\mathrm{NR}}, \ddot{A}_{\ell m}^{\mathrm{NR}}, \omega_{\ell m}^{\mathrm{NR}}, \dot{\omega}_{\ell m}^{\mathrm{NR}}, \ddot{\omega}_{\ell m}^{\mathrm{NR}}\right)$ at $t_{\text {extr }}^{\mathrm{NR}}(\nu)$. Then, for any value of $a_{6}^{c}$, we first compute the EOB dynamics, then we solve the linear system given by Eqs. (29a)-(29f) to obtain the NQC parameters $\left(a_{i}^{\ell m}, b_{i}^{\ell m}\right)$; and finally we iterate the procedure until $\left(a_{i}^{\ell m}, b_{i}^{\ell m}\right)$ converge at the fourth digit.

\section{Determining $a_{6}^{c}(\nu)$}

At this stage, the only freedom left in the model is the value of $a_{6}^{c}(\nu)$. Let us now explain how we investigated the phase difference $\Delta \phi^{\mathrm{EOBNR}}\left(a_{6}^{c}(\nu)\right)$ and used it to determine $a_{6}^{c}(\nu)$. Actually, we used a two-pronged approach towards studying $\Delta \phi^{\mathrm{EOBNR}}$. We first studied the $Q_{\omega}(\omega)$ function defined by the NR data, and compared it to the EOB-predicted one. Then, in a second step, we considered the time-domain phase difference $\Delta \phi^{\mathrm{EOBNR}}(t)$.

Let us start by explaining how we used the $Q_{\omega}(\omega)$ diagnostics to constrain the possible good values of $a_{6}^{c}(\nu)$. Since, as we explained above, we could extract from NR data a rather accurate estimate of $Q_{\omega}^{\mathrm{NR}}(\omega)$, we compared it to the value $Q_{\omega}^{\mathrm{EOB}}\left(\omega ; a_{6}^{c}(\nu)\right)$ predicted, for each value of $a_{6}^{c}(\nu)$, by EOB theory. Such a comparison (in the $q=2$ case) is illustrated in Fig. 7. The top panel of this figure shows the $\mathrm{EOB}-\mathrm{PN}$ and $\mathrm{NR}-\mathrm{PN}$ differences $\Delta Q_{\omega}^{X} \equiv Q_{\omega}^{\mathrm{X}}-Q_{\omega}^{3.5 \mathrm{PN}}$, where $X$ labels either EOB (for the three indicated values of $a_{c}^{6}$ ) or $\mathrm{NR}$, and $Q_{\omega}^{3.5 \mathrm{PN}}$ is the 3.5PN-accurate, Taylor-expanded expression given by Eq. (62). Note first that the black solid line, corresponding to $\mathrm{NR}-\mathrm{PN}$, shows that the current best $\mathrm{PN}$ knowledge of the intrinsic phasing function, $Q_{\omega}^{3.5 \mathrm{PN}}(\omega)$, differs from the NR result by a large amount, reaching $Q_{\omega}^{3.5 \mathrm{PN}}(\omega)-Q_{\omega}^{\mathrm{NR}}(\omega) \simeq-18$ at $M \omega_{2}=0.29$, which is close to merger. The corresponding integrated dephasing between $\mathrm{PN}$ and $\mathrm{NR}$,

$$
\Delta \phi^{\mathrm{PNNR}} \equiv \int_{\omega_{1}}^{\omega_{2}} d \ln \omega\left(Q_{\omega}^{3.5 \mathrm{PN}}(\omega)-Q_{\omega}^{\mathrm{NR}}(\omega)\right),
$$

accumulated from $M \omega_{1}=0.07$ to $M \omega_{2}=0.29$, is found to be equal to -11.72 radians.

By contrast to the $\mathrm{NR}-\mathrm{PN}$, or $\mathrm{EOB}-\mathrm{PN}$ differences displayed in the top panel of Fig. 7, its bottom panel displays the much smaller EOB-NR difference $\Delta Q_{\omega} \equiv Q_{\omega}^{\mathrm{EOB}}\left(\omega ; a_{6}^{c}(\nu)\right)-Q_{\omega}^{\mathrm{NR}}(\omega)$ for five different values of $a_{6}^{c}$. In addition, the shaded region represents the NR-NR difference $\Delta Q_{\omega}=Q_{\omega}^{\mathrm{NR}, \mathrm{N}=5}-Q_{\omega}^{\mathrm{NR}, \mathrm{N}=4}$, where where $N=5$ (respectively $N=4$ ) labels the numerical waveform with the highest (resp. medium) resolution [34]. The visual comparisons displayed in Fig. 7 are made quantitative in Table VI, which lists corresponding values of the EOB-NR phase difference over the frequency interval $M\left(\omega_{1}, \omega_{2}\right)=(0.07,0.29)$ obtained from 

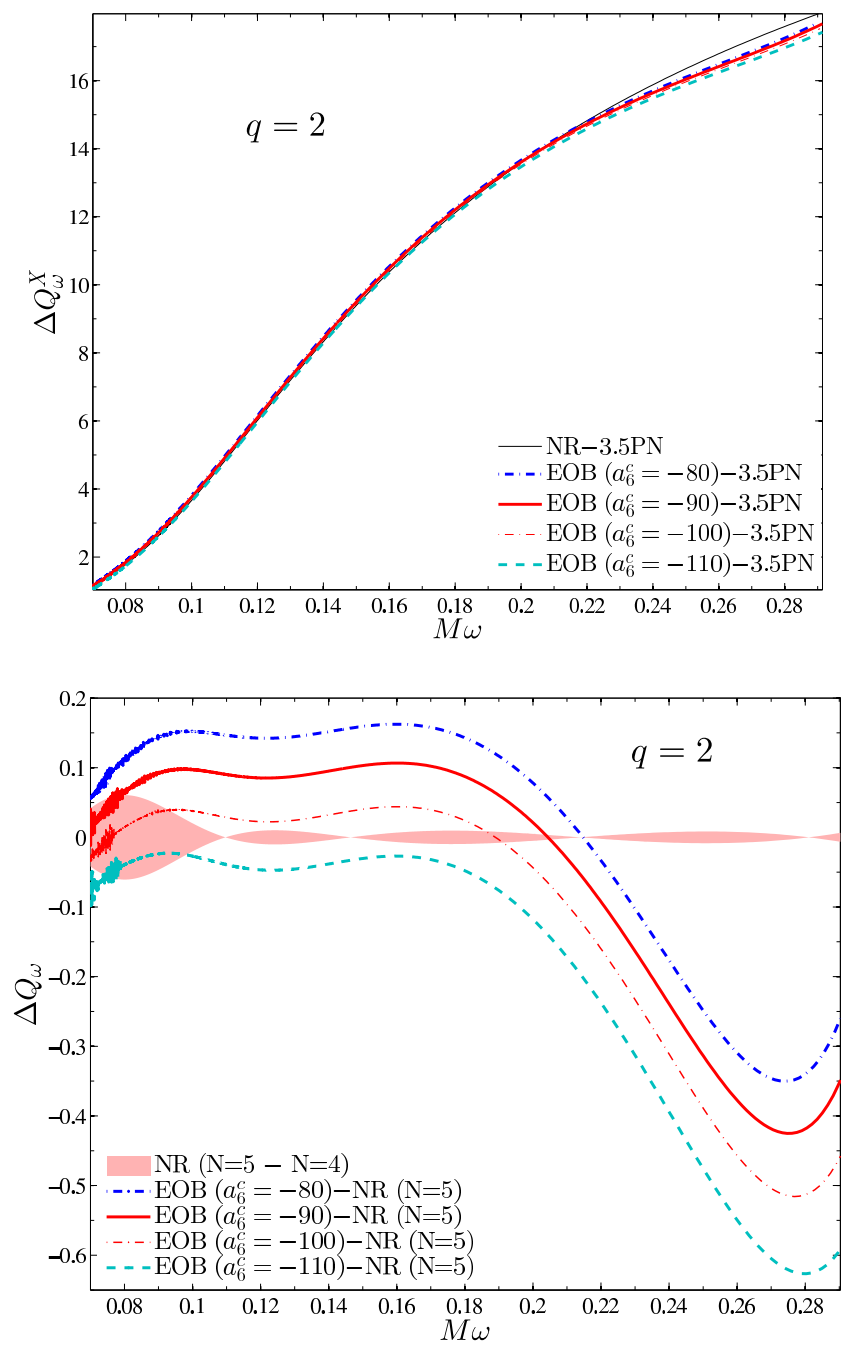

FIG. 7. (color online) Using the $Q_{\omega}(\omega)$ diagnostics to constrain the good values of $a_{6}^{c}(\nu)$. The figure refers to the case $q=2, \nu=2 / 9$. Top panel: difference between either $Q_{\omega}^{\mathrm{EOB}}$ or $Q_{\omega}^{\mathrm{NR}}$ and the 3.5PN-accurate, Taylor-expanded $Q_{\omega}^{3.5 \mathrm{PN}}$ given by Eq. (62). Bottom panel: the lines show the differences $\Delta Q_{\omega}=Q_{\omega}^{\mathrm{EOB}}-Q_{\omega}^{\mathrm{NR}, \mathrm{N}=5}$ for different values of $a_{6}^{c}$. The shaded region exhibits the difference $\Delta Q_{\omega}=$ $Q_{\omega}^{\mathrm{NR}, \mathrm{N}=5}-Q_{\omega}^{\mathrm{NR}, \mathrm{N}=4}$ where $N=4,5$ labels two different resolutions, respectively medium and high, of the NR data [34]. See text for further details.

the integral

$$
\Delta \phi \equiv \int_{\omega_{1}}^{\omega_{2}} d \ln \omega\left(Q_{\omega}^{\mathrm{EOB}}\left(\omega ; a_{6}^{c}(\nu)\right)-Q_{\omega}^{\mathrm{NR}}(\omega)\right) .
$$

Note that $M \omega_{2}=0.29$ approximately corresponds to the merger. These phase differences indicate that a good range of values of $a_{6}^{c}(2 / 9)$ is roughly between -90 and -100 . Within such a range, $\Delta \phi$ remains of the order of the NR phasing uncertainty as estimated in Ref. [28, 34] by comparing the two resolutions $N=4$ and $N=5$. Note that the small phase differences corresponding to $-100 \leq a_{6}^{c}(2 / 9) \leq-90$ result from a cancellation between positive and negative contributions to the above
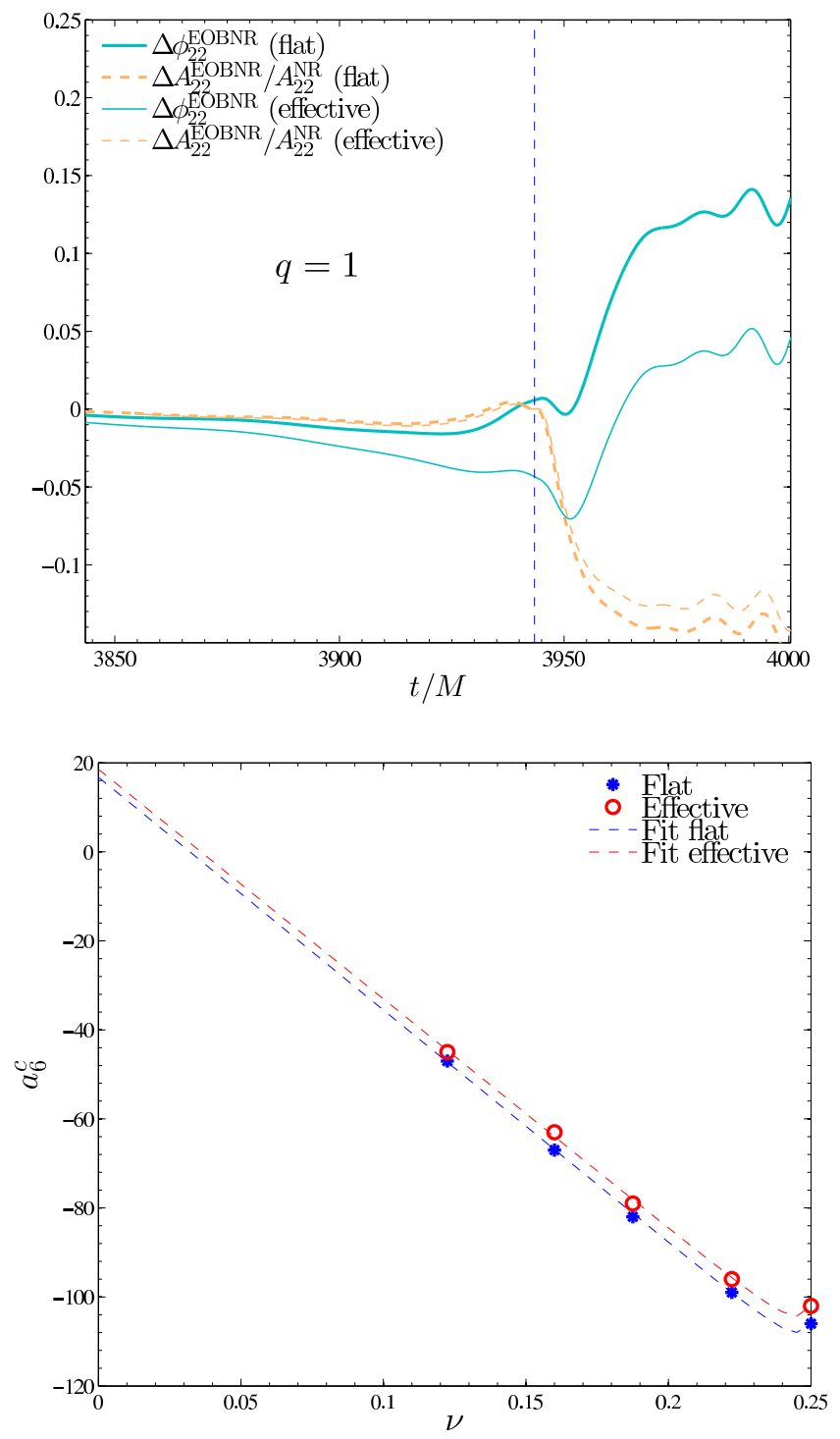

FIG. 8. (color online) Top panel: Illustrating the meaning of "flat" and "effective" EOB/NR phase differences around merger for $q=1(\nu=0.25)$. The flat phase difference is obtained here with $a_{6}^{c}(0.25)=-105.719$ from Eq. (73), while the effective one uses $a_{6}^{c}(0.25)=-101.876$, from Eq. (74). Bottom panel: "flat" and "effective" best values of $a_{6}^{c}$ and their analytical fits (dashed lines).

integral. However, a look at Fig 7 shows that within this range of $a_{6}^{c}$ the nonzero values of $\Delta Q_{\omega}$ remain of the order \pm 0.05 for most of the integration region. Such range of values of $\Delta Q_{\omega}$ is comparable to the numerical uncertainty on $Q_{\omega}$ (at least) during the inspiral, as illustrated by the shaded region in the figure. Note indeed that the frequency $M \omega=0.1$ is reached only $150 M$ before merger (cf. bottom left panel of Fig. 10). Note also that the frequency interval $0.2 \leq M \omega_{22} \leq 0.3$ (where the top panel of Fig. 7 shows visible differences, made quantitative in the bottom panel) only corresponds to the last $25 \mathrm{M}$ before merger. [The GW frequency 0.2 approximately cor- 

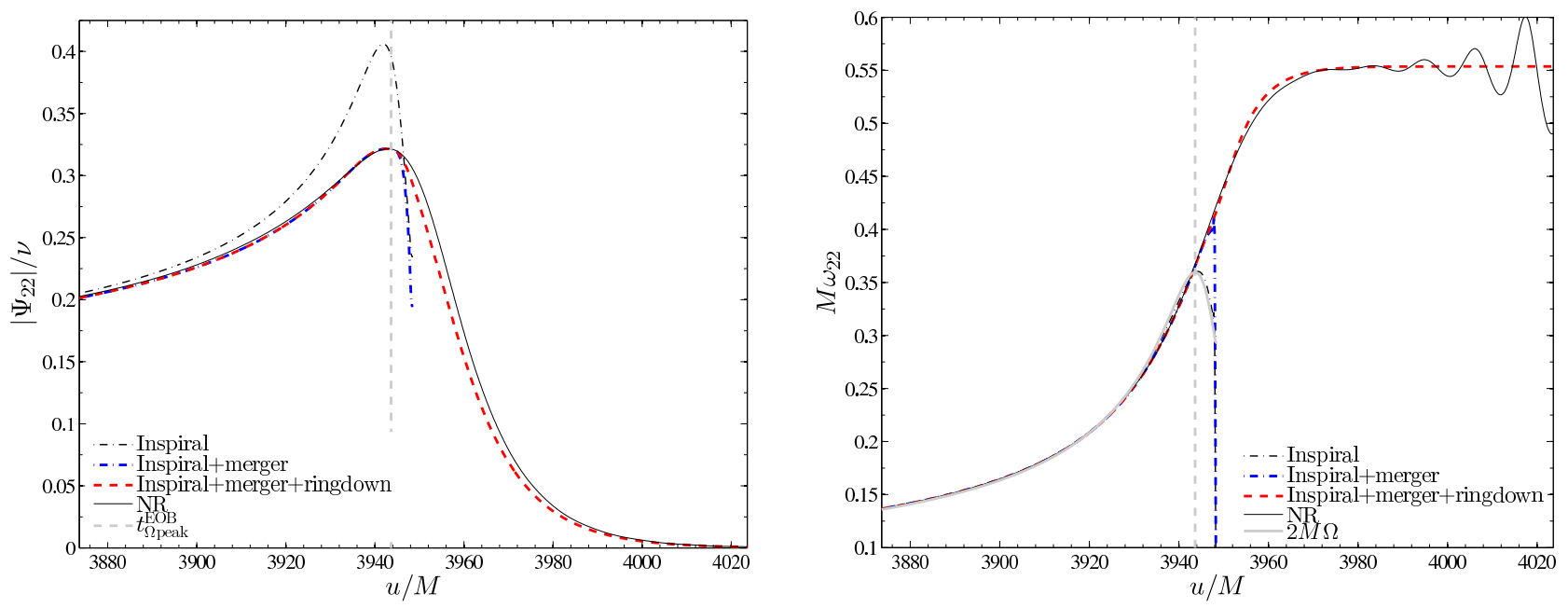

FIG. 9. (color online) Illustrating the effect of the NQC factor on the "bare", inspiral EOB waveform (equal-mass case): modulus (left panel) and frequency (right panel).

TABLE VI. Mass ratio $q=2$ : phase difference $\Delta \phi=\phi^{\mathrm{EOB}}-$ $\phi^{\mathrm{NR}}$ accumulated between frequencies $\omega_{1}=0.07$ and $\omega_{2}=$ 0.29 versus $a_{6}^{c}$ as obtained using Eq. (70).

\begin{tabular}{cc}
\hline \hline$a_{6}^{c}$ & $\Delta \phi[\mathrm{rad}]$ \\
\hline-80 & +0.0810 \\
-90 & -0.0010 \\
-100 & -0.0909 \\
-110 & -0.1942 \\
\hline \hline
\end{tabular}

TABLE VII. Best values of $a_{6}^{c}$ selected according to the behavior of the $\Delta \phi=\phi^{\mathrm{EOB}}-\phi^{\mathrm{NR}}$ phase difference around merger time.

\begin{tabular}{cccc}
\hline \hline$q$ & $\nu$ & $a_{6}^{c}$ (flat) & $a_{6}^{c}$ (effective) \\
\hline 1 & 0.25 & -106 & -103 \\
2 & $0 . \overline{2}$ & -99 & -96 \\
3 & 0.1875 & -82 & -79 \\
4 & 0.1600 & -67 & -63 \\
6 & 0.1224 & -47 & -45 \\
\hline \hline
\end{tabular}

responds to the adiabatic LSO crossing, i.e. the end of the quasi-adiabatic inspiral].

This analysis based on the $Q_{\omega}$ diagnostics selects, for each value of the mass ratio $(q=1,2,3,4,6)$, a range of good values of $a_{6}^{c}(\nu)$, which then needs to be confirmed and refined by directly comparing the time-domain phase evolution of the EOB waveform to the NR one. We have done such an analysis by considering, for each value of $a_{6}^{c}(\nu)$ within the above range, the phase evolution from the beginning of the simulation up to merger, and also after merger, during ringdown. The comparison up to merger only depends on the choices of $t_{\text {extr }}^{\mathrm{NR}}(\nu)$ and $a_{6}^{c}(\nu)$, while the comparison during the subsequent ringdown also depends on the choices made in attaching QNMs to the NQC-corrected pre-merger signal. The time-domain phasing comparison allowed us to close up, for each value of $\nu$, on a more precisely determined value of $a_{6}^{c}(\nu)$ (with an uncertainty of order unity). Actually, depending on the criterion we put on the quality of the $\mathrm{EOB} / \mathrm{NR}$ phase agreement, the resulting best values of $a_{6}^{c}(\nu)$ are slightly different. However, in all the cases we have explored, we found that the good, $\nu$-dependent values of $a_{6}^{c}$ were approximately lying along a straight line.

We choose $a_{6}^{c}$ according to the following two criterions: on the one hand, we can require that the time-domain phase difference (after alignment) $\Delta \phi^{\operatorname{EOBNR}}\left(t ; a_{6}^{c}(\nu)\right)$ remains near zero in as flat a manner as possible up to merger. In this case, the price to pay for this is that the subsequent, somewhat coarse QNM attachment defined by the current EOB prescriptions, will cause, after merger and during ringdown, the EOB-NR phase difference $\Delta \phi^{\mathrm{EOBNR}}\left(t ; a_{6}^{c \text { flat }}(\nu)\right)$ to jump to positive values of order $\sim+0.15$ rad (more about this below). On the other hand, one can also look for a more "effective" description of the phasing where we allow $\Delta \phi^{\text {EOBNR }}$ to take slightly negative values just before merger, but to jump to smaller values $\sim+0.05$ rad after merger (see more details below). The so obtained corresponding good values of $a_{6}^{c}$ are listed in Table VII. The bottom panel of Fig. 8 plots these values versus $\nu$. One sees that, for both the effective and flat cases, they approximately lie along a straight line. However, as evidenced by these plots, a linear fit to $a_{6}^{c}(\nu)$ does not give an accurate representation of the points when the $\nu=0.25$ value is taken into account. Before discussing a way to fit such a behavior, let us note that the top panel of Fig. 8 displays, for $q=1$, the phase differences for the "flat" and "effective" values 

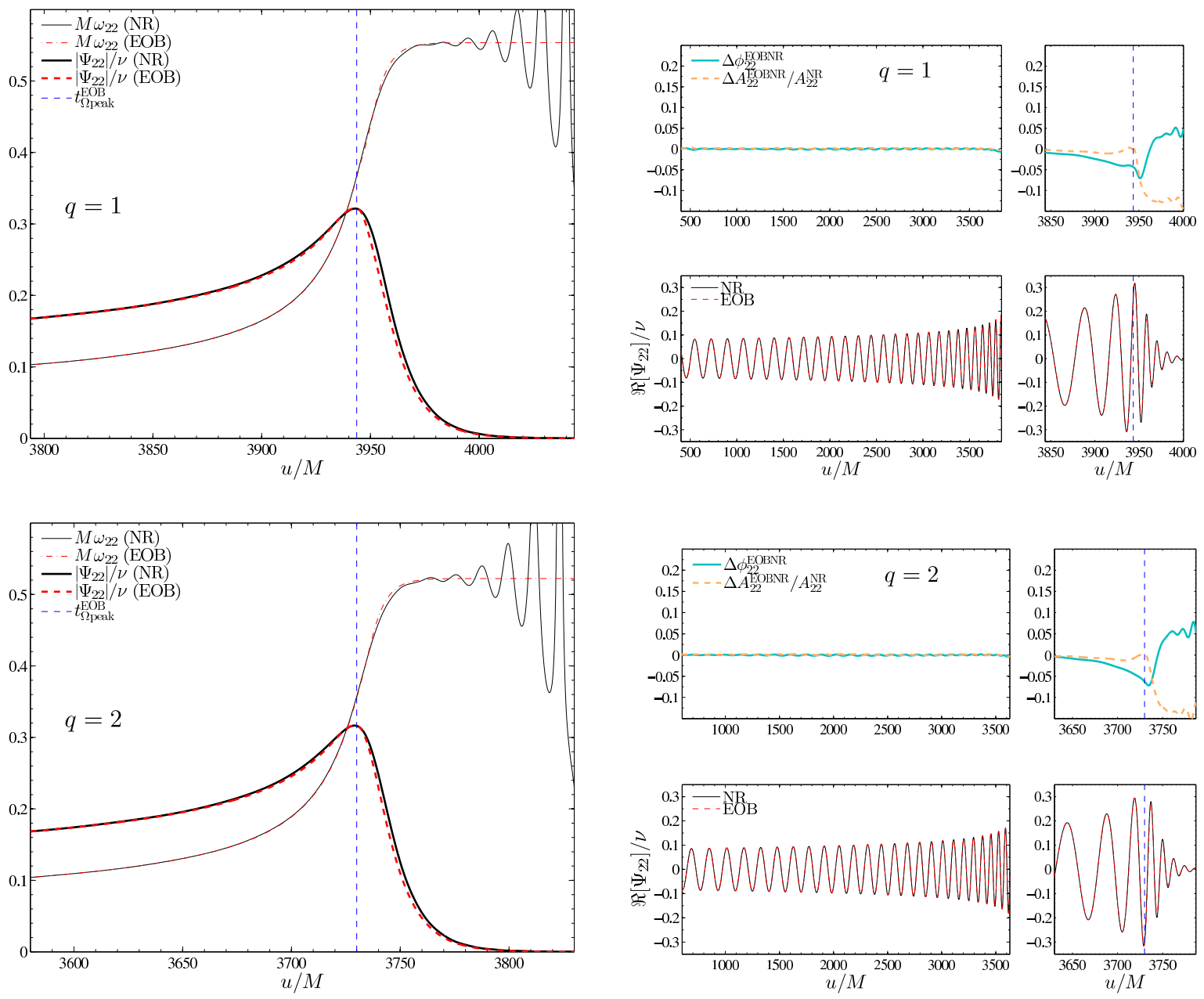

FIG. 10. (color online) Comparison between EOB and NR (Zerilli-normalized) waveforms for mass ratios $q=1,2$. Left panels: amplitude and frequency. In the right panels, each subplot shows the phase and amplitude differences between the EOB and NR waveform (top) and the real part of $\Psi_{22}$ (bottom). The time axis is the NR one: $u=t^{\mathrm{NR}}$. The EOB waveform has been time- and phase-shifted so as to minimize the EOB - NR phase difference for frequencies $M \omega<0.1$. The vertical dashed lines mark the $t_{\Omega \text { peak }}^{\mathrm{EOB}}$ crossing time.

of $a_{6}^{c}(0.25)$. The same behavior, with very similar phase differences, is found for all other mass ratios.

Let us come back to the issue of constructing an analytical fit for the behavior of the functions $a_{6}^{c}(\nu)$ exhibited in the bottom panel of Fig. 8. We checked that the use of a global linear fit for the values of $a_{6}^{c}(\nu)$ would give unacceptably large phase differences $(>0.1 \mathrm{rad})$ accumulated up to merger. This suggests the need of using a fitting function which deviates from a linear function of $\nu$ only in a rather limited interval $0 . \overline{2}<\nu \leq 0.25$. There are many ways to construct such fits. Here, as a first attempt (to be possibly improved in future work), we have used the following, factorized, mostly-linear, functional form

$$
a_{6}^{c}(\nu)=[a+b(1-4 \nu)] \tilde{s}(c ; \nu),
$$

where $\tilde{s}$ denotes a localized (when the parameter $c$ is much smaller than one) correction to the linear behavior parametrized by $a$ and $b$ :

$$
\tilde{s}(c ; \nu) \equiv\left(1+\frac{c}{(0.26-\nu)^{2}}\right)^{1 / 2} .
$$

We have determined sufficiently accurate values of the parameters $(a, b, c)$ by fitting the the values of $a_{6}^{c}$ listed in Table VII in two steps. [For simplicity, we fixed the location of the pole in the function $\tilde{s}^{2}(\nu)$ to the fiducial value $\nu=0.26$.] First $(a, b)$ were determined by fitting only the $q=(2,3,4,6)$ data in Table VII to a straight line. The raw data were then divided by the outcome of the fit and the resulting ratios were further fitted against the factor of Eq. (71) so as to determine c. Applying this fitting procedure, we find $(a, b, c)^{\text {flat }}=$ $\left(-114.006,130.774,-1.352 \times 10^{-5}\right)$ for the flat choices of $a_{6}^{c}$ and $(a, b, c)^{\text {effective }}=(-110.467,129.022,-1.468 \times$ $10^{-5}$ ) for the effective choices of $a_{6}^{c}$. Rounding up these numbers, we summarize our search of a "flat" $a_{6}^{c}(\nu)$ by 

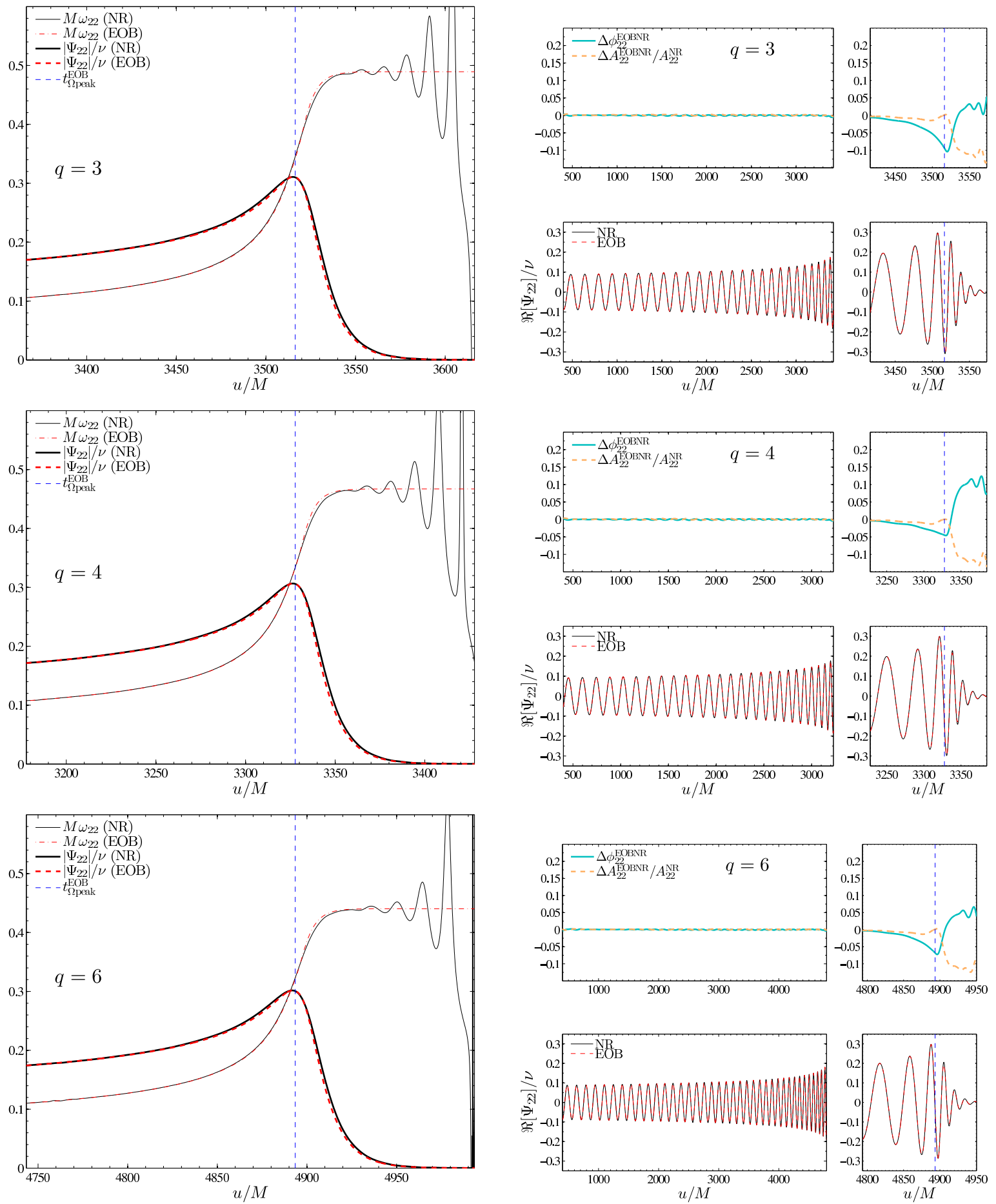

FIG. 11. (color online) Comparison between EOB and NR (Zerilli-normalized) waveforms for mass ratios $q=3,4,6$. Left panels: amplitude and frequency. In the right panels, each subplot shows the phase and amplitude differences between the EOB and NR waveform (top) and the real part of $\Psi_{22}$ (bottom). The vertical dashed lines mark the $t_{\Omega \text { peak }}^{\mathrm{EOB}}$ crossing time.

the following analytical expression

$$
a_{6}^{c \text { flat }}(\nu)=[-114+131(1-4 \nu)] \tilde{s}\left(-1.4 \times 10^{-5} ; \nu\right) .
$$

For the effective description of the phasing we found in- stead

$$
a_{6}^{c}(\nu)=[-110.5+129(1-4 \nu)] \tilde{s}\left(-1.5 \times 10^{-5} ; \nu\right) .
$$

This is one of the central results of our work, and one of the most important pieces in the NR-completion of our 
EOB model.

In conclusion, we propose to define the NR completion of our EOB model by adopting the analytical expressions (68) and (74) for defining, respectively, $t_{\text {extr }}^{\mathrm{NR}}(\nu)$ and $a_{6}^{c}(\nu)$. In addition, we found that the following QNMattachment choices define a reasonably accurate ringdown completion of the EOB waveform: $N=5 \mathrm{QNM}$ modes, and $\Delta^{\text {comb }}=0.7 M$. In the following, we shall illustrate the comparison of the EOB multipolar waveform defined by these choices to the corresponding NR multipolar waveform.

\section{Values of the NQC parameters $\left(a_{i}^{\ell m}, b_{i}^{\ell m}\right)$}

Before doing so, let us recall that, for each mass ratio, we must determine (by iteration) the NQC parameters $\left(a_{i}, b_{i}\right)$ defined by the above choices (using given $\mathrm{NR}$ data). In Table $\mathrm{V}$ we list, for the mass ratios $q=(1,2,3,4,6)$ and for multipoles $(2,2),(2,1),(3,3)$, $(3,2)$, the values of the $\left(a_{i}^{\ell m}, b_{i}^{\ell m}\right)$ 's that define the NQC corrections to the bare inspiral-plus-plunge EOB waveform. [When $q=1$ there are no entries for $\ell m=(2,1)$ and $(3,3)$, because these modes are identically zero in this case for symmetry reasons.] We will discuss below the issue of replacing the information contained in this table by $\nu$-dependent fitting formulas.

\section{E. Effect of the NQC factor on the EOB waveform}

Let us first illustrate how the NQC factor modifies the purely inspiral EOB waveform. The $q=1$ case is considered in Fig. 9: modulus (left panel) and frequency (right panel). Similar results are obtained for any other mass ratio (see also Ref. [55] for the test-mass limit). We show together: (i) the purely inspiral waveform, i.e., Eq. (16) without the NQC factor $\hat{h}_{\ell m}^{\mathrm{NQC}}$ (dash-dotted, thin line, black online); (ii) the inspiral+merger waveform, including the NQC factor (dash-dotted and thick line, blue online); (iii) the extended EOB waveform, including the ringdown part (thick, solid line, red online); and the NR waveform (thin, solid line, black online). As noted already in Ref. [55] the most striking feature of this plot is that the pure inspiral EOB waveform modulus peaks (after alignment as explained in Sec. V F) just $\sim 1.4 M$ before the peak of the NR modulus. On the other hand, its amplitude is about $20 \%$ larger than the $\mathrm{NR}$ one ${ }^{12}$. Note that the largish difference in amplitude is very effectively corrected by the NQC factor. In order to reduce

\footnotetext{
12 Such a behavior follows from our use of $x=v_{\varphi}^{2}$ as argument in $\rho_{22}(x)$. As noted in Fig. 2 of Ref. [28], the different choice $x \rightarrow \Omega^{2 / 3}$ (which is however not physically justified during the plunge), makes the EOB waveform peak considerably earlier (by $6.2 M)$ than $\mathrm{NR}$, but with an amplitude much closer to the NR one $(\approx-0.23 \%$ smaller $)$.
}

the amplitude and displace it to the right we need a NQC factor that, near merger, is smaller than one and growing. This is what $n_{2}$ succeeds in doing thanks to its shape, as illustrated in Appendix A. This explains why the values of the NQC parameter $a_{2}^{22}$ are the dominant ones, see Table $\mathrm{V}$. By contrast, if one has to increase the amplitude and displace it to the right (as was needed in Ref. [28] because of the use of the argument $\Omega^{2 / 3}$ in $\left.\rho_{22}(x)\right)$, one needs a NQC factor which, near merger, is larger than one and growing, as, for instance, our $n_{1}$, Eq. (28a).

\section{F. Comparison between the $\ell=m=2 \mathrm{NR}$ and EOB waveforms}

Let us now present the results of the comparison between the dominant quadrupolar $((\ell, m)=(2,2))$ $\mathrm{NR}$ waveform, and the corresponding NR-completed EOB waveform introduced in this work. For each mass ratio among $q=(1,2,3,4,6)$, Figs. 10-11 compare the EOB and NR modulus and frequency (left panels), the real parts of the waveforms (right panels, bottom) and also show the phase difference $\Delta \phi^{\mathrm{EOBNR}} \equiv \phi^{\mathrm{EOB}}-\phi^{\mathrm{NR}}$ and the relative amplitude difference $\Delta A^{\mathrm{EOBNR}} / A^{\mathrm{NR}} \equiv\left(A^{\mathrm{EOB}}-A^{\mathrm{NR}}\right) / A^{\mathrm{NR}}$ (right panels, top). The vertical dashed line present in all panels marks the location of the peak of the EOB orbital frequency, $t_{\Omega \text { peak }}^{\mathrm{EOB}}$. These time-domain comparisons are done by suitably determining a relative time and phase shift between the two phases $\phi_{22}^{\mathrm{NR}}\left(t^{\mathrm{NR}}\right)$ and $\phi_{22}^{\mathrm{EOB}}\left(t^{\mathrm{EOB}}\right)$. These shifts are estimated by minimizing the time integral of the square of the phase difference on a time interval corresponding to a given frequency interval $\left[M \omega_{L}, M \omega_{R}\right]$. Following Refs. [28, 67] , we perform this waveform alignment on the long inspiral phase. Note that, in doing so, we do not enforce the constraint that $t_{\text {extr }}^{\mathrm{NR}}$ corresponds to $t_{\Omega \text { peak }}^{\mathrm{EOB}}$. However, the EOB/NR agreement is so good up to merger that such an early-inspiral alignment succeeds in realizing, a posteriori, a near coincidence between $t_{\text {extr }}^{\mathrm{NR}}$ and $t_{\Omega \text { peak }}^{\mathrm{EOB}}$. For instance, we find that, for $q=1$, $t_{\text {extr }}^{\mathrm{NR}}-t_{\Omega \text { peak }}^{\mathrm{EOB}} \simeq-0.13 M$. The right limit of the frequency for each mass ratio is $M \omega_{R}=0.1$. The left bounds are $M \omega_{L}=(0.035,0.035,0.035,0.044,0.045)$.

These figures indicate an excellent EOB/NR agreement in phasing and in modulus from the early inspiral up to merger. The remaining disagreements are well within the nominal error bar on numerical data. Actually, the only estimate of the numerical error on the phasing of these numerical data that is available in the literature is a rather conservative one that is done by taking the difference between the highest and the medium resolution. This procedure gives uncertainties that are very small during the inspiral phase $(<0.01 \mathrm{rad})$ and small, though not negligible, in the late plunge phase up to merger $(\sim 0.1-0.3 \mathrm{rad}$, depending on the mass ratio) [34]. A less conservative NR error estimate might be 

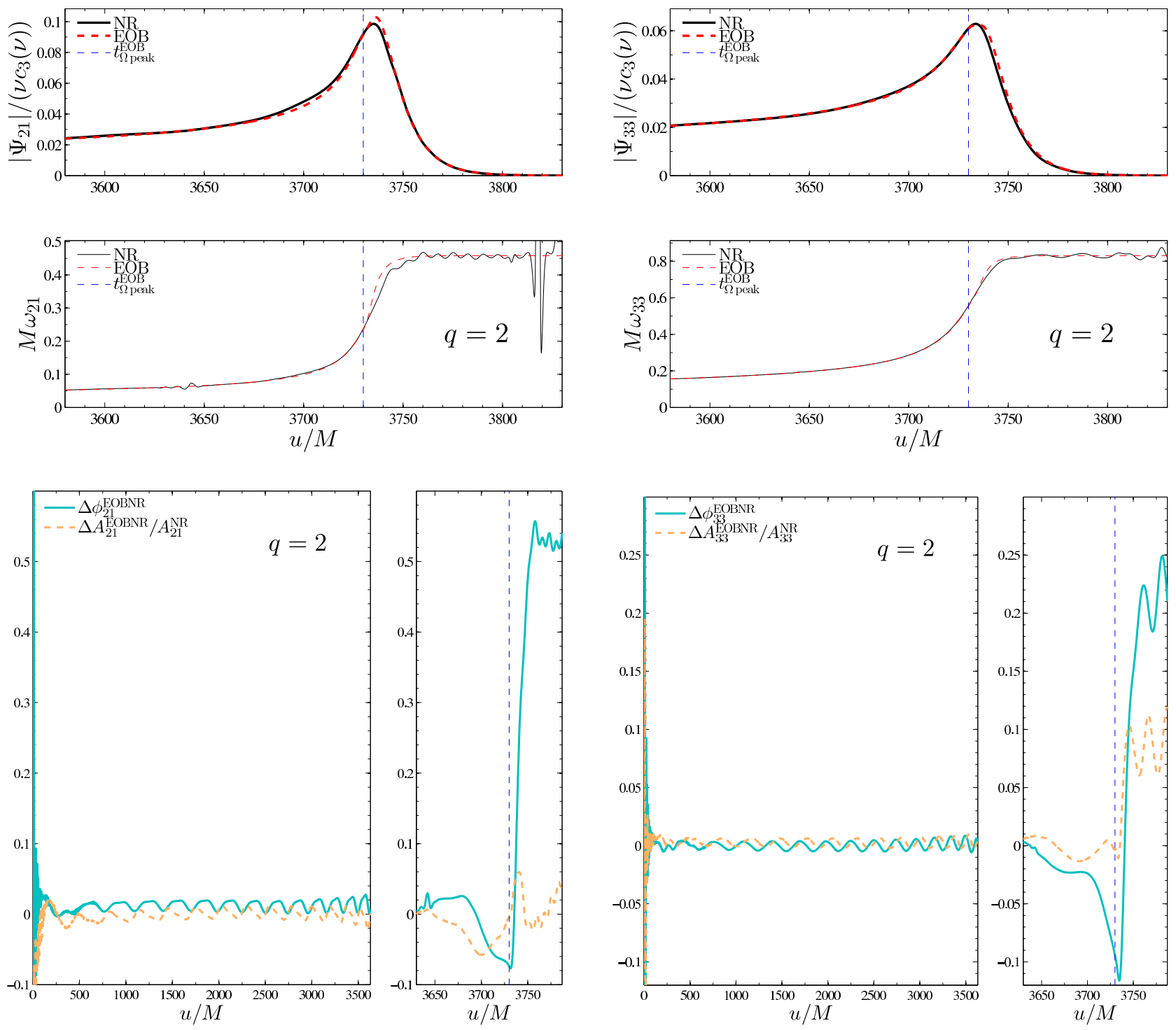

FIG. 12. (color online) Subdominant multipoles, $\ell=2, m=1$ (left panels) and $\ell=m=3$ (right panels) Comparison between EOB model and NR (Zerilli-normalized) waveform for mass ratio $q=2$. Top: amplitudes and frequencies. Bottom panels: amplitude and phase differences. The vertical dashed lines mark the $t_{\Omega \text { peak }}^{\mathrm{EOB}}$ crossing time.

smaller by (at least) a factor two ${ }^{13}$. Keeping this in mind, it is remarkable that our EOB model, with the very simple law for $a_{6}^{c}(\nu)$ given in Eq. (74) is able to reproduce all numerical data within $\lesssim 0.06$ radians at merger.

Let us also emphasize the very good agreement between the moduli before and at merger (see the top-right inset in the right-panels of Figs. 10-11), though they exhibit a visible difference during the subsequent ringdown. The good agreement before merger is an improvement with respect to previous works $[22,28,45]$ that is due to a combination of effects coming both from the use of an

13 We thank Harald Pfeiffer and Luisa Buchman for informing us of this more realistic estimate of the NR errors. improved analytical EOB model, from a new choice of the basis of NQC functions $n_{i}$, and from the choice of an NQC determination point which differs from the maximum of the amplitude. [ Note that such an agreement before merger is also comparable to the one obtained by Taracchini et al. [29] with an EOB model that is rather different from the one discussed here]. Let us also note that, as already mentioned, we have, on purpose, chosen effective values of $a_{6}^{c}(\nu)$ causing the phase difference $\Delta \phi^{\mathrm{EOBNR}}$ to dip towards negative values $\sim-0.05 \mathrm{rad}$ just before merger, before jumping towards positive values of order +0.05 or +0.1 rad during ringdown. Such a behavior ensures a good average phase agreement during the entire process. Had we instead chosen the slightly different "flat" values of $a_{6}^{c}(\nu)$, Eq. (73), they would have led 

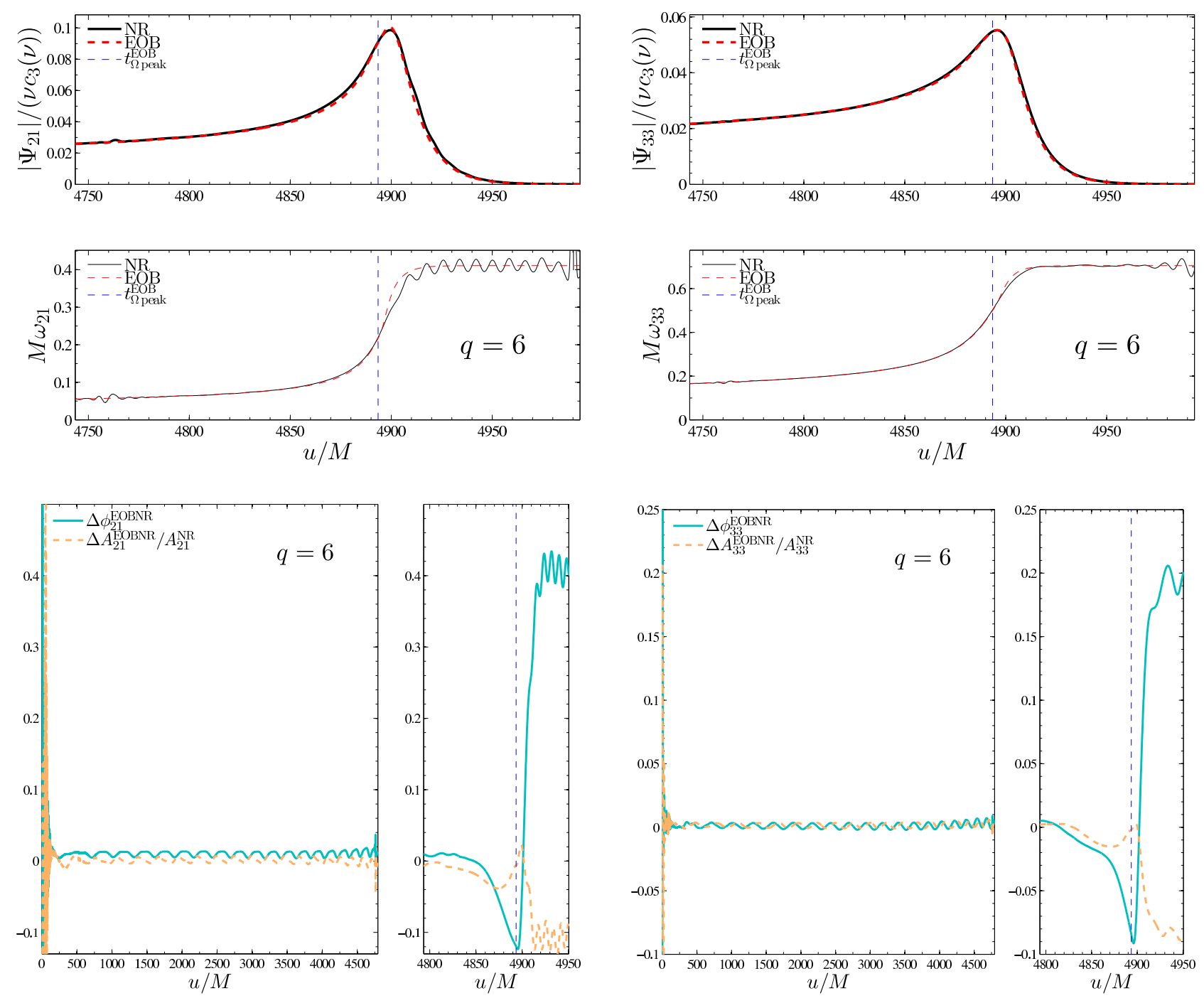

FIG. 13. (color online) Subdominant multipoles, $\ell=2, m=1$ (left panels) and $\ell=m=3$ (right panels) Comparison between EOB model and NR (Zerilli-normalized) waveform for mass ratio $q=6$. Top: amplitudes and frequencies. Bottom panels: amplitude and phase differences. The vertical dashed lines mark the $t_{\Omega \text { peak }}^{\mathrm{EOB}}$ crossing time.

to a near perfect phase agreement up to merger. However, the price for doing so would then have been the presence of a larger global phase disagreement (of order $\sim+0.15 \mathrm{rad})$, due to a positive jump in $\Delta \phi^{\mathrm{EOBNR}}$ after merger, and during ringdown. We note that such a positive jump $\sim+0.15 \mathrm{rad}$ in $\Delta \phi^{\mathrm{EOBNR}}$ is consistent with the study, done in Ref. [28], of the intrinsic error in $\Delta \phi^{\text {EOBNR }}$ coming from the procedure of QNM attachment itself. This indicates that more work should be devoted towards improving the current EOB technique for attaching QNMs onto the inspiral-plus-plunge waveform.

\section{G. Subdominant multipoles}

Up to now, our study has only considered the dominant quadrupolar $\ell=m=2$ waveform. Let us now compare some of the subdominant multipolar waveforms. We consider here the $\ell=2, m=1$ and $\ell=m=3$ subdominant waveforms, for the two mass ratios $q=2$ and $q=6$ (similar results were obtained for $q=3$ and $q=4$ ). We limit ourselves to such a partial comparison here to show the capability of the EOB model, as it was defined above, to get the main characteristics of the subdominant multipoles, without introducing ad hoc modifications, or tuning further parameters. At the end of this section we will also mention some results for the $\ell=3, m=2$ multipole.

In Figs. 12-13 we compare, for the two mass ratios $q=2$ and $q=6$, the NR and EOB frequency and modulus for the two subdominant multipoles $\ell=2, m=1$ and $\ell=m=3$ (top panels) as well as the phase and amplitude differences (bottom panels). We use the same matching interval as for the $\ell=m=2$ mode, i.e. 

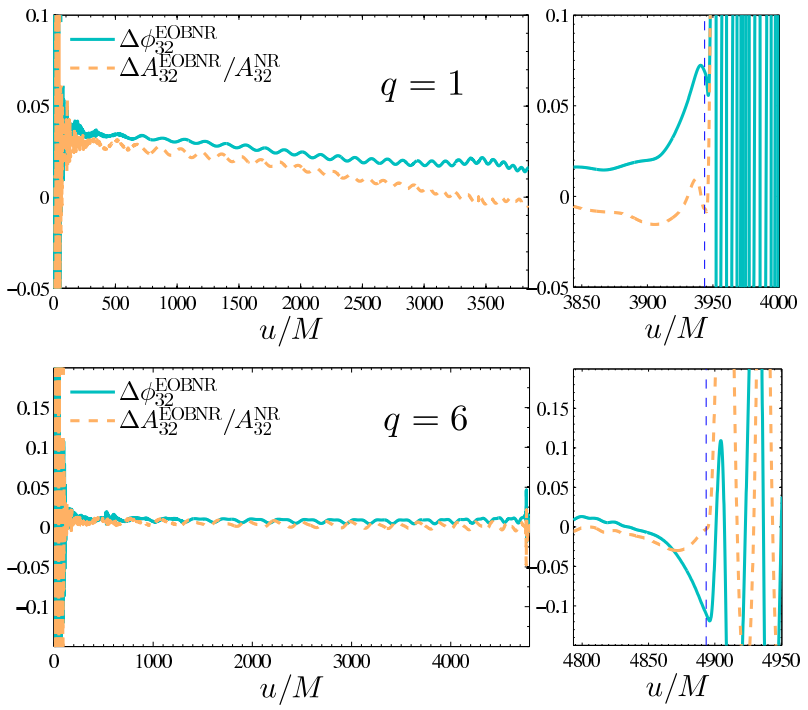

FIG. 14. (color online) Subdominant multipole $\ell=3, m=2$ : phase difference for $q=1$ (top panel) and $q=6$ (bottom panel). The vertical dashed lines mark the $t_{\Omega \text { peak }}^{\mathrm{EOB}}$ crossing time.

$\Delta^{\text {match }}=0.7 M$, and the same number of QNM modes, i.e. $N=5$. Note the good agreement of the moduli in all cases, both up to merger, and during ringdown [In the $A_{21}, q=2$ case the multiple crossings between the NR and EOB moduli may be due to inaccuracies in the NR waveform.] Note also the good agreement, up to merger, of the frequencies, in all cases, and the good agreement of the frequency of the $(3,3)$ mode after merger, and during ringdown. The only case which is slightly less successful is the discrepancy between the EOB frequency and the NR frequency in the $\ell=2, m=1$ case for both mass ratios (compare with Ref. [28], but note we have not introduced here any ad hoc treatment of the the $\ell=2$, $m=1$ case.) Namely, the EOB frequency of the $(2,1)$ mode shoots up, just after merger, a bit faster than its NR counterpart. In turn, such a frequency difference builds up a phase difference after merger. This is illustrated in the bottom panels of the figure, which shows the phase differences $\Delta \phi_{21}^{\mathrm{EOBNR}}$ (left) and $\Delta \phi_{33}^{\mathrm{EOBNR}}$ (right) as functions of time during the entire simulation. Note that the dephasing is remarkably small up to merger for both multipoles, and then accumulates a dephasing $\Delta \phi_{21}^{\mathrm{EOBNR}} \sim 0.5 \mathrm{rad}$ (and $\Delta \phi_{33}^{\mathrm{EOBNR}} \sim 0.15 \mathrm{rad}$ ) during the ringdown.

Let us emphasize that the phase difference $\Delta \phi_{21}^{\mathrm{EOBNR}}(t)$ plotted in the bottom panels of Figs. 12-13 has been computed without introducing any new arbitrariness, neither in time, nor in phase, in comparing the two phase evolutions. Indeed, the least-squares alignment procedure of the NR and EOB dominant $(2,2)$ waveforms has determined both a shift in time, say $\tau_{22}$, and a phase shift, say $\alpha_{22}$, connecting them. The time shift $\tau_{22}$ determines the (a priori unknown) connection between the two time variables $t^{\mathrm{NR}}$ and $t^{\mathrm{EOB}}$, and should therefore be used in comparing the time evolutions of all the other physical quantities, and in particular the subdominant multipoles. The case of the phase shift $\alpha_{22}$ is similar, but with a difference. Indeed, in our case (with a common, preferred $z$ axis given by the total angular momentum of the sytem) the only a priori unknown angular difference between $\mathrm{NR}$ and $\mathrm{EOB}$ is a rotational shift, by some angle $\beta$, connecting the NR basis of tensorial spherical harmonics to the corresponding EOB basis. This common angle $\beta$ then introduces a phase shift in all the various $\ell m$ multipoles simply given by

$$
\alpha_{\ell m}=m \beta,
$$

independently of $\ell$. As this result applies in particular to $\alpha_{22}$ (which is determined modulo $2 \pi$ by the alignment of the $(2,2)$ waveforms), we see that the phase shifts in the subdominant multipoles are determined to be

$$
\alpha_{\ell m}=\frac{m}{2} \alpha_{22} \text { modulo } m \pi
$$

In addition to this phase shift, there might be extra phase shifts due to the use of different conventions in defining the phase of the tensorial spherical harmonics. Such phase conventions differ at most by multiples of $\pi / 2$, corresponding to powers of $i$. In other words, we can always write that $\alpha_{\ell m}=\frac{m}{2} \alpha_{22}$ modulo $\pi / 2$, which is sufficient for unambiguously computing $\Delta \phi_{\ell m}^{\mathrm{EOBNR}}$ for all subdominant multipoles. This absence of phase-shift ambiguity in $\Delta \phi_{\ell m}^{\mathrm{EOBNR}}$ makes it all the more remarkable that, in the $(2,1)$ case, the phase difference $\Delta \phi_{21}^{\mathrm{EOBNR}}$ plotted in Fig. 12 (for $q=2$ ) and Fig. 13 (for $q=6$ ) stays very small up to merger.

Let us finally comment on Fig. 14, were we show the phase difference one gets for the $\ell=3, m=2$ multipole, for the two representative cases $q=1$ (top panel) and $q=6$ (bottom panel). The figure, again, illustrates a rather good consistency between EOB and NR up to merger. The differences after merger are mostly due to our simplified description of the ringdown (see Appendix A of Ref. [28] for a detailed analysis of the structure of the $(3,2)$ ringdown waveform).

We leave to future work a more detailed analysis of the subdominant multipoles, and the investigation of possible ways of improving their EOB representation, in case the slight dephasing exhibited in Figs. 12-13 for the $(\ell, m)=(2,1)$ multipole happens to significantly degrade the faithfulness of the complete EOB waveform (summed over all multipoles).

\section{STRUCTURE OF THE EOBNR RADIAL POTENTIAL $A(u)$ AND ITS CONNECTION WITH OTHER RESULTS}

One of the most important nonperturbative dynamical knowledge acquired in this work by comparing EOB 


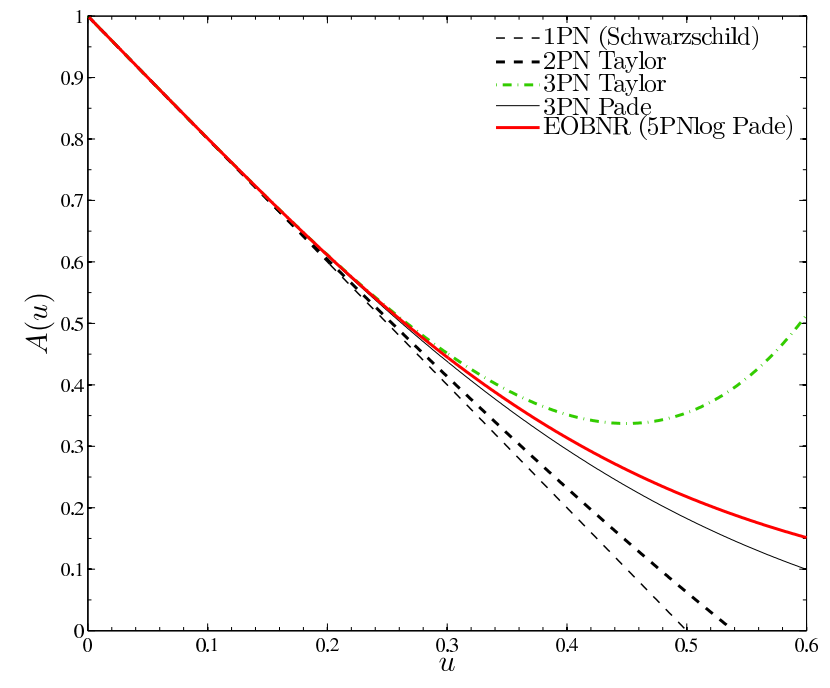

FIG. 15. (color online) Contrasting various estimates of the $A(u ; \nu)$ function. in the equal-mass case, $\nu=0.25$. The plot shows the $1 \mathrm{PN}, 2 \mathrm{PN}$ and $3 \mathrm{PN}$ Taylor-expanded versions of $A(u ; 0.25)$; its $3 \mathrm{PN}$-accurate Padé resummed form as well as the EOBNR one (5PN accurate with logarithmic terms and $a_{5}^{c}=23.5, a_{6}^{c}(0.25)=-101.876$, as per Eq. (74)).

predictions to the Caltech-Cornell-CITA simulations concerns the function $A(u ; \nu)$. We recall that $A(u ; \nu)$ is the main radial potential of the EOB Hamiltonian, and represents the time-time component of the effective EOB metric: $A(u ; \nu)=-g_{00}^{\text {eff }}(R)$. In the test-mass limit, $\nu \rightarrow 0$, the effective metric is the Schwarzschild metric, so that $\lim _{\nu \rightarrow 0} A(u ; \nu)=1-2 u \equiv 1-2 G M /\left(R c^{2}\right)$. We saw above that NR data selected, in the strong-field domain, an $A$ function given by Eq. (10) with $a_{5}^{c}=23.5$ and $\left.a_{6}^{c}(\nu)=[-110.5+129(1-4 \nu)] \tilde{s}\left(-1.5 \times 10^{-5} ; \nu\right)\right)$. Let us now discuss some properties of this NR-informed EOB potential (or simply EOBNR potential) and its connection with other relevant results.

\section{A. Global shape of $A^{\mathrm{EOBNR}}(u ; \nu)$ as a function of $u$, and comparison with previous purely analytical estimates}

As a first orientation, we contrast in Fig. 15 various estimates of the function $A(u ; \nu)$ in the equal-mass case, i.e. $\nu=0.25$. Our NR-informed estimate (5PN$\log$-Padé resummed and with $a_{5}^{c}=23.5$ and $a_{6}^{c}(\nu)=$ $\left.[-110.5+129(1-4 \nu)] \tilde{s}\left(-1.5 \times 10^{-5} ; \nu\right)\right)$ is shown as a thick solid line (red online), i.e. the second line from the top. The dashed bottom line represents the 1PNaccurate estimate of $A$, which happens to coincide with the simple Schwarzschild-metric result $A^{\mathrm{Schw}}(u)=1-2 u$. [Indeed, in Eq. (7) there are no terms of order $u^{2}$ corresponding to the 1PN level.] The thicker dashed line just above this 1PN estimate represents the Taylor-expanded 2PN estimate, i.e. Eq. (7) taken up to the term $\mathcal{O}\left(u^{3}\right)$ included. The upper dashed line represents the Taylorexpanded $3 \mathrm{PN}$ estimate of $A(u ; \nu)$, as given by Eq. (7) up to the term $\mathcal{O}\left(u^{4}\right)$ included. Finally, the thin solid line (black online) just below the NR-completed 5PNlog Padé curve is the Padé-resummed estimate of the analytically known 3PN result, which was proposed by Damour, Jaranowski and Schäfer [3] in 2000, i.e. five years before NR simulations started yielding information about the strong-field dynamics of binary black holes. It is remarkable that the latter simple 3PN-Padé estimate is rather close to the best current NR-informed estimate: (i) it is numerically quite close to it if one considers values $u \lesssim 0.3$ which are already beyond the last stable orbit, and therefore are crossed during the plunge; and (ii) even in the very strong field domain $0.3 \lesssim u \lesssim 0.6$ (where the merger occurs) the 3PN-Padé estimate is a much better approximation to $A^{\mathrm{EOBNR}}(u ; \nu)$ than any of its standard PN approximants. This closeness explains the success of the simple Padéed 3PN $A$ function in agreeing with several recent NR studies of dynamical aspects of close black hole binaries [26,33], and confirms the effectiveness of using Padé approximants to improve the strong-field behavior of Taylor approximants.

\section{B. Detailed study of the $\nu$-dependence of $A^{\mathrm{EOBNR}}(u ; \nu)$}

The comparison of the previous subsection has indicated that an accurate description of the gravitational wave emission of coalescing binary black holes requires a very precise determination of the shape of $A(u ; \nu)$ in the very strong-field domain $u \gtrsim 0.3$ (i.e. $R \lesssim 3 G M / c^{2}$ ). Let us zoom on the detailed shape of the $A$ function in the strong-field domain by focusing on the properties of the associated $a$ function, defined by writing

$$
A(u ; \nu) \equiv 1-2 u+\nu a(u ; \nu) .
$$

The Taylor expansion of this small- $a$ function starts as

$$
a(u ; \nu)=2 u^{3}+\left(\frac{94}{3}-\frac{41}{32} \pi^{2}\right) u^{4}+O\left(u^{5} \ln u\right) .
$$

Note that the $\nu$ dependence of $a(u ; \nu)$ is only contained in the $O\left(u^{5} \ln u\right)$ remainder term. In order to zoom on the $\nu$ dependence of $a(u ; \nu)$ it is then useful, following Ref. [48], to normalize the $a$ function by its LO PN behavior, $a^{2 P N}(u ; \nu)=2 u^{3}$, i.e. to consider the $\hat{a}(u ; \nu)$ function defined as

$$
\hat{a}(u ; \nu) \equiv \frac{a(u ; \nu)}{2 u^{3}} \equiv \frac{A(u ; \nu)-(1-2 u)}{2 \nu u^{3}} .
$$

In the upper panel of Fig. 16, we plot the values of the EOBNR $\hat{a}(u ; \nu)$ functions for the values of $\nu$ corresponding to the five mass ratios we used in our EOB/NR comparisons above, namely $q=1,2,3,4,6$, as well as the EOBNR predicted $\hat{a}$ curves corresponding to $q=10$, to $q=100$ and also to $q=\infty$, i.e. to the $\nu=q /(q+1)^{2} \rightarrow 0$ 
limit of $\hat{a}^{\mathrm{EOBNR}}(u ; \nu)$. The (red online) round markers on the curves indicate the EOB-defined, light-ring locations, i.e. the solutions of the equation $\left(u^{2} A(u)\right)^{\prime}=0$ (see Table VIII for the precise numbers). In addition, we have also indicated the recently derived (GSFcomputed) "exact" value of the $\operatorname{limit}_{\nu \rightarrow 0} \hat{a}(u ; \nu)[48]$ (using their best analytical fit). In the bottom panel of Fig. 16 we plot the corresponding values of the products $\nu a^{\operatorname{EOBNR}}(u ; \nu)=2 \nu u^{3} \hat{a}^{\mathrm{EOBNR}}(u ; \nu)$, i.e. the corresponding differences of $A^{\operatorname{EOBNR}}(u ; \nu)$ away from its test-mass limit, i.e. $A^{\mathrm{EOBNR}}(u ; \nu)-A^{\mathrm{Schw}}(u)$, where $A^{\mathrm{Schw}}(u)=$ $1-2 u=\lim _{\nu \rightarrow 0} A^{\operatorname{EOBNR}}(u ; \nu)$. This shows again how the physics of the GW emission by coalescing black hole binaries depends on fine features in the $A$ potential. Note how, as $\nu$ decreases, $\hat{a}(u ; \nu)$ monotononically increases, in a way which is qualitatively compatible with the shape of the limiting GSF result $\hat{a}(u ; 0)=\lim _{\nu \rightarrow 0} \hat{a}(u ; \nu)$. [The latter limiting GSF shape has a singularity at $u=1 / 3$, which is probably smoothed out by higher-order corrections in $\nu$ around $\nu=0$. See [48] for a detailed discussion of the origin of this singularity, and its probable fictitious character.] Though the $\nu \rightarrow 0$ limit of $\hat{a}^{\mathrm{EOBNR}}(u ; \nu)$ (which is a polynomial in $u$, with logarithmic coefficients) does not coincide with the exact $\mathcal{O}(\nu)$ GSF result, it stays quite close to it up to $u \lesssim 0.2$. It is interesting in this respect to point out that the $\nu \rightarrow 0$ limit of our NR fitted $a_{6}^{c}(\nu)$, given by Eq. $(54)$, is $a_{6}^{c}(0)=+18.4979 \approx+18.5$. This is completely different from the true Taylor value $a_{6}^{c \text { Taylor }}(0)=-131.72(1)$ [38]. However, it has the same sign and order of magnitude as the effective value obtained above, in Eq. (12), by requiring compatibility with the GSF determination of LSO precession for $\nu \rightarrow 0$. This shows a reasonable compatibility between two effective determinations of $a_{6}^{c}(0)$ in the strong field regime.

Note also, on the bottom panel, how the behavior of the corresponding contribution to the $A$ potential, i.e. the product $\nu a(u ; \nu)$, seems to tend continuously (though maybe not uniformly) towards zero as $\nu \rightarrow 0$. This bottom panel suggests that the $q=10$ case should be thought of as belonging to the class of the normal comparable-mass cases $q=\mathcal{O}(1)$. One needs $q$ 's of order at least $\mathcal{O}(100)$ to belong to the class of extreme-massratio binaries. The EOBNR potential derived here has anyway been tuned to the physics of comparable-mass binaries with $1 \leq q \leq 6$. As we knew (from Ref. [48]) that the $\nu \rightarrow 0$ limit of the (exact) $A$ potential was (probably) mildly singular, and as we are mainly interested in describing the physics of comparable-mass systems, we did not attempt to incorporate in the $A$ function too much of the information contained in its $\nu \rightarrow 0$, GSF limit. In our work above, we only incorporated some information about the $\nu \rightarrow 0$ limit of the $4 \mathrm{PN}$ coefficient $\lim _{\nu \rightarrow 0} a_{5}^{c}(\nu)$. But, as we shall discuss next, this was mainly done as a practical way of reducing the number of unknowns to be fitted to NR data.
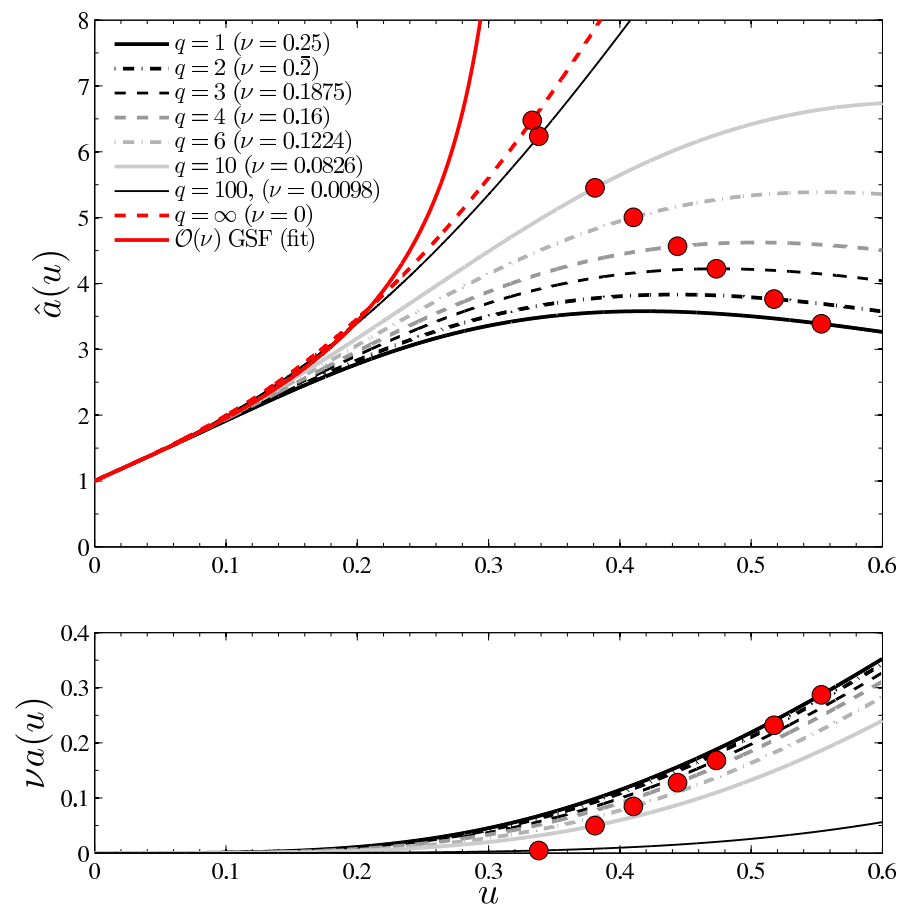

FIG. 16. (color online) Top panel: Behavior of the EOBNR $\hat{a}(u)$ function defined in Eq. (79) with $a_{5}^{c}=23.5$ and $a_{6}^{c}(\nu)=$ $[-110.5+129(1-4 \nu)] \tilde{s}\left(-1.5 \times 10^{-5} ; \nu\right)$. The red line shows the $\nu=0$ function as obtained from the fit of GSF data [48]. Bottom panel: the difference $A^{\text {EOBNR }}(u ; \nu)-A^{\text {Schw }}(u)$ with $A^{\text {Schw }}(u)=1-2 u$. For each value of $\nu$, the marker indicates the EOB-defined adiabatic light ring location.

TABLE VIII. EOB-defined adiabatic light-ring (LR) and laststable-orbit (LSO) locations for $a_{5}^{c}=23.5$ and $a_{6}^{c}=[-110.5+$ $129(1-4 \nu)] \tilde{s}\left(-1.5 \times 10^{-5} ; \nu\right)$.

\begin{tabular}{cccccc}
\hline \hline$q$ & $\nu$ & $r_{\mathrm{LR}}$ & $u_{\mathrm{LR}}$ & $r_{\mathrm{LSO}}$ & $u_{\mathrm{LSO}}$ \\
\hline 1 & 0.25 & 1.8067 & 0.5535 & 4.5108 & 0.2217 \\
2 & $0 . \overline{2}$ & 1.9324 & 0.5175 & 4.6964 & 0.2129 \\
3 & 0.1875 & 2.1119 & 0.4735 & 4.9226 & 0.2031 \\
4 & 0.1600 & 2.5223 & 0.4440 & 5.0962 & 0.1962 \\
6 & 0.1224 & 2.4366 & 0.4104 & 5.3235 & 0.1878 \\
10 & 0.0826 & 2.6240 & 0.3811 & 5.5529 & 0.1801 \\
$\infty$ & 0 & 3.0000 & $0 . \overline{3}$ & 6.000 & $0.1 \overline{6}$ \\
\hline \hline
\end{tabular}

\section{On the "equivalence classes" of the $A(u)$ potential}

References $[6,22]$ found, for the $q=1$ case, that there was a strong degeneracy between the two parameters entering a $5 \mathrm{PN}$-accurate Padé representation of the $A$ function, say $a_{5}^{c}$ and $a_{6}^{c}$. This was confirmed for other values of $q$ in Ref. [28]. This finding leads to the idea that the good values of $a_{5}^{c}$ and $a_{6}^{c}$ can be organized in "equiva- 
lence classes" of quasi-interchangeable values of the pairs $\left(a_{5}^{c}, a_{6}^{c}\right)$. An explicit way of constructing these equivalence classes was indicated in [35]: it consists in defining the equivalence class of some given pair $\left(a_{5}^{c(0)}, a_{6}^{c(0)}\right)$ as the set of pairs $\left(a_{5}^{c}, a_{6}^{c}\right)$ such that the $u$-derivative $A^{\prime}\left(u ; \nu ; a_{5}^{c}, a_{6}^{c}\right)$ of the $A$ function, evaluated at some fiducial strong-field point, say $u_{b}$ (the value $u_{b} \simeq 0.215$ was suggested there), takes the same value at $\left(a_{5}^{c}, a_{6}^{c}\right)$ and at $\left(a_{5}^{c(0)}, a_{6}^{c(0)}\right)$. In equations

$$
A^{\prime}\left(u_{b} ; \nu ; a_{5}^{c}, a_{6}^{c}\right)=A^{\prime}\left(u_{b} ; \nu ; a_{5}^{c(0)}, a_{6}^{c(0)}\right),
$$

or, equivalently,

$$
a^{\prime}\left(u_{b} ; \nu ; a_{5}^{c}, a_{6}^{c}\right)=a^{\prime}\left(u_{b} ; \nu ; a_{5}^{c(0)}, a_{6}^{c(0)}\right) .
$$

When working, as we do here, with the normalized function $\hat{a}(u ; \nu)$, we could alternatively define these equivalence classes as level sets (in the space of pairs $\left(a_{5}^{c}, a_{6}^{c}\right)$ ) of $\hat{a}^{\prime}\left(u_{b} ; \nu ; a_{5}^{c}, a_{6}^{c}\right)$, or even, simply, of $\hat{a}\left(u_{b} ; \nu ; a_{5}^{c}, a_{6}^{c}\right)$. Evidently, all those possible "definitions" lead (when one changes the fiducial value $u_{b}$, and/or the considered function $a^{\prime}, \hat{a}^{\prime}, a$, etc.) to different equivalence classes. However, because of the properties of the $A$ function, one checks that, as long as one bases one's definition on the value of $A$ or some related function in the strong-field region, this leads, to a good approximation, to a numerically rather well-defined equivalence class of $\left(a_{5}^{c}, a_{6}^{c}\right)$ pairs. This is illustrated in Fig. 17. This figure shows (for the case $q=1$ ) that our NR-tuned preferred values $\left(a_{5}^{c(0)}, a_{6}^{c(0)}\right)=(23.5,-101.876)$ define a $\nu a(u)$ function which can be very nearly reproduced by using other pairs of $\left(a_{5}^{c}, a_{6}^{c}\right)$ values, namely $(0,220)$, $(5,125)$, or $(10,40)$. The upper panel shows together, versus $u$, the functions $\nu a\left(u ; \nu ; a_{5}^{c}, a_{6}^{c}\right)$, for $q=1$, i.e. $\nu=$ 0.25 , and for the four different pairs of parameters values $\left(a_{5}^{c}, a_{6}^{c}\right)=(23.5,-101.876),(10,40),(5,125),(0,220)$. The upper panel illustrates that these five different functions are indistinguishable by eye. The bottom panel of the figure zooms on the differences away from our standard choice $\left(a_{5}^{c(0)}, a_{6}^{c(0)}\right)=(23.5,-101.876)$, i.e. it plots $\nu \Delta a\left(u ; \nu ; a_{5}^{c}, a_{6}^{c}\right) \equiv A\left(u ; \nu ; a_{5}^{c}, a_{6}^{c}\right)-$ $A(u ; \nu ; 23.5,-101.876)$. For any choice of the parameters, these differences are of the order $10^{-4}$. Note that we have not used, here, any precise, level-set type, criterion for selecting the pairs equivalent to our preferred value, but we have selected them by simple trial and error, until we could reduce the (maximum) difference to the smallest level we could find . This smallest level was $O\left(10^{-4}\right)$. The reason why such a level of deviation is small enough for our purpose can be seen by turning back to our analysis above, when we were fixing the fiducial value $a_{5}^{c}=23.5$, and then tuning the value of $a_{6}^{c}$ for the EOB phasing to best agree with the NR one. In that case, as is clear from the number of digits we were giving in Table VII above for $a_{6}^{c}$ (before fitting them), we found that the "good" values of $a_{6}^{c}$ were determined, roughly, within an uncertainty $\delta a_{6}^{c}=\mathcal{O}(1)$. Such an uncertainty
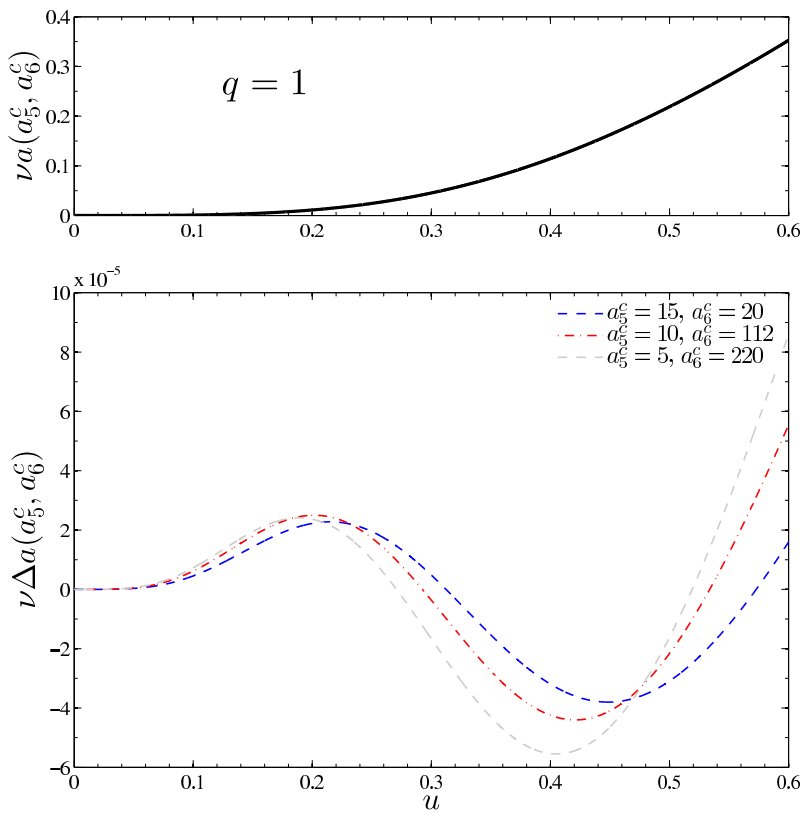

FIG. 17. (color online) Elements of the equivalence class of $\hat{a}\left(a_{5}^{c}, a_{6}^{c}\right)$ functions for $q=1$. The bottom panel shows the fractional difference with our favorite choice $a_{5}^{c}=23.5$, $a_{6}^{c}(0.25)=-101.876$.

on the good value of $a_{6}^{c}$ (for the fixed $a_{5}^{c}=23.5$ ) entails a corresponding uncertainty on the value of the function $A\left(u ; a_{5}^{c}, a_{6}^{c}\right)$ of order $\delta A\left(u ; a_{5}^{c}, a_{6}^{c}\right) \sim \partial A\left(u ; a_{5}^{c}, a_{6}^{c}\right) / \partial a_{6}^{c}$. The latter quantity is found to increase with $u$, and to reach a value of order $0.8 \times 10^{-4}$ when $u$ takes the lightring value $u_{\mathrm{LR}} \simeq 0.55$ (for $q=1$ ). In conclusion, a possible variation in the $A(u)$ function of $L_{\infty}$ norm $\sim 10^{-4}$, for $0 \leq u \leq u_{\mathrm{LR}}$, is a reasonable way of defining the equivalence class of $A(u)$, and Fig. 17 shows that one can indeed, starting from the (analytically fitted) values $\left(a_{5}^{c(0)}, a_{6}^{c(0)}\right)=(23.5,-101.876)$, find a (relatively thin) strip of values of $\left(a_{5}^{c}, a_{6}^{c}\right)$ along which the 5PN Padéd function $A^{\mathrm{EOBNR}}\left(u ; a_{5}^{c}, a_{6}^{c} ; \nu\right)$ stays within such an equivalence class.

Though here we focus only on the $q=1$ case, similar classes of equivalence of $\hat{a}$ functions exist for any mass ratio. In summary, this exercise confirms that we were justified in a priori fixing the value of $a_{5}^{c}$. Finally, the important fact is that NR data allow one to directly determine the $A(u ; \nu)$ function itself, essentially independently of the chosen "representative" $\left(a_{5}^{c}, a_{6}^{c}\right)$ within some equivalence strip in the $\left(a_{5}^{c}, a_{6}^{c}\right)$ plane. This determination of the $A^{\mathrm{EOBNR}}(u ; \nu)$ function is exemplified on Fig. 16 (keeping in mind the invisible deviations plotted in the upper panel of Fig. 17). 


\section{Comparison between the present determination of $A^{\mathrm{EOBNR}}(u ; \nu)(5 \mathrm{PN}$ with logs), with previous estimates (5PN without logs).}

The present work is the first EOB work to include logarithmic terms in a comparison with NR data. Let us now compare our final NR-aided determination of such an $A$ function (with logarithmic terms) to the $5 \mathrm{PN}$-accurate $A$ functions (without logarithmic terms) used in previous EOB works [6, 12, 22, 27-29]. In particular, Ref. [22], using a $5 \mathrm{PN}$-accurate $A\left(a_{5}^{c}, a_{6}^{c} ; \nu\right)$ function (without logs), exploited a previous version of the $q=1$ Caltech-CornellCITA numerical waveform to find a banana-like region of good values in the $\left(a_{5}^{c}, a_{6}^{c}\right)$ plane such that the phase difference between EOB and NR waveform through inspiral, plunge and merger was $<0.02 \mathrm{rad}$. The values $a_{5}^{c}=-6.37$ and $a_{6}^{c}=50$ lie in the middle of this good region and have been used extensively in subsequent EOB work [26, 27, 31, 42]. [By contrast Ref. [22] actually used the values $a_{5}^{c}=0$ and $a_{6}^{c}=-20$ which lie on the boundary of the good region.] The analog, banana-shaped equivalence classes in the $\left(a_{5}^{c}, a_{6}^{c}\right)$ plane corresponding to other values of $q$ were then first investigated in Ref. [28]. [The latter reference basically used the same conceptual structure as Ref. [22] with some technical differences.] Ref. [28] found a very good agreement between EOB and NR waveforms with an $A$ function defined by the following choices

$$
a_{5}(\nu)=-5.828-143.5 \nu+447 \nu^{2}, \quad a_{6}=184 .
$$

More recently, Barausse and Buonanno [12] introduced a differently resummed $A$ function, which is $3 \mathrm{PN}$ accurate and does not contain the $4 \mathrm{PN}$ and $5 \mathrm{PN}$ logarithmic contributions used in the present work. Their resummation does not rely on a Padé approximant, but imposes by hand the presence of a horizon, by factoring out of $A(u)$ a binomial of the form $1-2(1-K(\nu) \nu) u+a^{2}(1-K(\nu) \nu)^{2} u^{2}$ . [Here, $a$ is a Kerr-like spin parameter, which vanishes in the non-spinning case considered here.] The flexibility parameter $K(\nu)$, which effectively parametrizes $4 \mathrm{PN}$ and higher contributions, was then calibrated in Ref. [29] against Caltech-Cornell-CITA non-spinning waveforms (for $q=1,2,3,4,6$ ), with the result:

$$
K(\nu)=1.447-1.715 \nu-3.246 \nu^{2} .
$$

In Fig. 18 we consider the two mass ratios $q=1$ and $q=6$ and for each mass ratio we compare four different $\hat{a}(u)$ curves, namely: (i) the log-containing $5 \mathrm{PN}$-accurate one determined in this work ("EOBNR$\log "$ with $a_{5}^{c}=+23.5$, and $a_{6}^{c}(\nu)=[-110.5+129(1-$ $\left.4 \nu)] \tilde{s}\left(-1.5 \times 10^{-5} ; \nu\right)\right)$; (ii) the log-less $5 \mathrm{PN}$-accurate one of [22] (with $a_{5}^{c}=-6.3$ and $a_{6}^{c}=50$ ); (iii) the log-less $5 \mathrm{PN}$-accurate one of [28], see Eq. (82); and (iv) the (logless) 3PN-accurate Barausse-Buonanno [12] one, $\hat{a}^{\mathrm{BB}}(u)$, for the value of the adjustable parameter, $K(\nu)$, cited above [29].

The figure shows that while the first three different analytical descriptions seem to be visually close for the

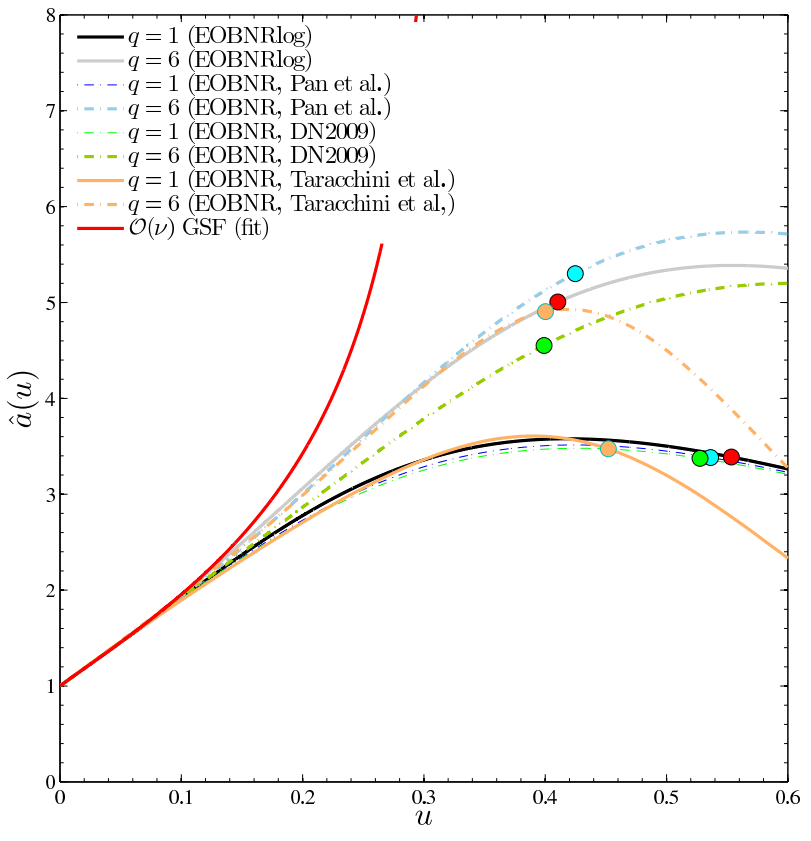

FIG. 18. (color online) Comparing $\hat{a}$ functions for different, $5 \mathrm{PN}$-accurate, EOBNR-completed models. The markers indicate the location of the EOB-defined adiabatic light-ring for each curve. See text for explanations.

equal-mass case, $q=1$, they exhibit visible differences in the $q=6$ case. However, we have seen above that only differences of order $10^{-4}$ in the $A$ function can be considered as being negligibly small. When computing the differences $\Delta A^{X}(u ; \nu) \equiv A^{X}-A^{\text {EOBNRlog }}$ for the two labels $X=$ DN2009, Ref. [22] and $X=$ Pan et al, Ref. [28], one finds that, for $q=1, \Delta A^{X}(u)$ is a monotonically decreasing function of $u$ which reaches values of order $\simeq-0.004$ for $X=$ DN2009 and $\simeq-0.0025$ for $X=$ Pan et al when $u \simeq 0.5$, i.e., close to the corresponding adiabatic lightring position. Such differences are therefore quite significant on the $10^{-4}$ scale of the equivalence classes of $A$ functions exhibited in Fig. 17. In the $q=6$ case the corresponding differences taken at $u \simeq 0.4$, close to the adiabatic light-ring position, are $\simeq-0.006$ for $X=$ DN2009 and $\simeq+0.003$ for $X=$ Pan et al.. Again these differences are quite significant. Note however that for $u \leq 0.3$ the log-less model of [28], Eq. (82), (which had been tuned to the same $q=6 \mathrm{NR}$ data as ours) stays quite close to our present log-containing model $\left(\Delta A=2 \times 10^{-4}\right)$.

Let us finally discuss the comparison with the (log-less) Barausse-Buonanno $\hat{a}^{\mathrm{BB}}(u)$ function calibrated by Taracchini et al. [29] (orange line online, solid for $q=1$, dashed for $q=6$ ). Figure 18 shows that up to its own light ring (marked by an orange circle on the curves) this function stays rather close to our EOBNRlog one with $a_{5}^{c}=+23.5$, and $a_{6}^{c}(\nu)=[-110.5+129(1-4 \nu)] \tilde{s}\left(-1.5 \times 10^{-5} ; \nu\right)$. The differences are however so large that $\hat{a}^{\mathrm{BB}}$ cannot be considered to be part of the equivalence class of EOBNRlog in the sense discussed above. More precisely, we 
find that the difference between the respective $A$ potentials varies, roughly, between \pm 0.01 for $q=1$ and between \pm 0.005 for $q=6$. This is two orders of magnitudes larger than the $10^{-4}$ level that we used above to define the equivalence class (see Fig. 17 and corresponding text). Despite this, one finds that the adiabatic LSO orbital frequencies predicted by the two potentials are very close. For $q=1$, we have $\Omega_{\mathrm{LSO}}^{\mathrm{EOBNRlog}}=0.0993$ and $\Omega_{\mathrm{LSO}}^{\mathrm{BB}}=0.1010$ (i.e. $\mathrm{a} \sim 2 \%$ difference), and for $q=6$ we obtain $\Omega_{\mathrm{LSO}}^{\text {EOBNRlog }}=0.0801$ and $\Omega_{\mathrm{LSO}}^{\mathrm{BB}}=0.0797$. In addition, we see on the figure that the difference $\hat{a}^{\mathrm{BB}}(u)-$ $\hat{a}^{\mathrm{EOBNRlog}}(u)$ oscillates in sign around zero, so that the phasing defined by $\hat{a}^{\mathrm{BB}}(u)$ can be expected to agree, on average, with that defined by $\hat{a}^{\text {EOBNRlog }}(u)$. We have performed a quantitative check of this expectation by considering the phasing during the quasi-adiabatic inspiral, which is rather directly related to the conservative part of the dynamics and thereby to the $A(u)$ function. More precisely, we computed, for each $A(u)$ potential, the adiabatic phasing along the sequence of EOB circular orbits. This phasing is best measured by the (adiabatic) $Q_{\omega}^{\text {adiabatic }}(\omega) \equiv-5 /(24 \nu) x^{-1} \partial_{x} j_{0}(\hat{f}(x))^{-1}$ function. Here, $\omega=2 \Omega$ is the adiabatic GW frequency, $x=\Omega^{2 / 3}, \hat{f}$ is the resummed, Newton-normalized, energy flux as introduced in Eq. (37) above, and $j_{0}$ is the angular momentum along the sequence of EOB circular orbits defined by Eq. (51) for a given $A$ potential. We then focus on the difference $\Delta Q_{\omega}(\omega)=Q_{\omega}^{\mathrm{EOBNRlog}}(\omega)-Q_{\omega}^{\mathrm{BB}}(\omega)$.

Inspection of the $\Delta Q_{\omega}(\omega)$ function more or less confirms the conclusion drawn from the comparison between the $\hat{a}(u)$ functions in Fig. 18. More precisely, we find that: for $q=1$ it basically averages around zero up to the LSO, varying between \pm 0.5 in a frequency range $\Delta \omega=(0.03,0.2)$; on the contrary, for $q=6$ the same function is negative and monotonically decreasing over the frequency interval $\Delta \omega=(0.03,0.16)$, reaching the value $\sim-4.4$ at $\omega=0.16$. As explained in Sec. III above where the $Q_{\omega}(\omega)$ function was introduced, the usefulness of this phasing diagnostic is that its integral over $\ln \omega$ directly gives the GW phase as a function of frequency. Correspondingly the integral $\Delta \phi=\int_{0.03}^{\bar{\omega}_{\mathrm{LSO}}} \Delta Q_{\omega} d \ln (\omega)$ yields the relative dephasing (here estimated in the adiabatic approximation) between the waveforms corresponding to the choice of two different $A$ potentials, which is accumulated between the initial frequency $\omega=0.03$ and the average LSO frequencies, say $\bar{\omega}_{\mathrm{LSO}}=0.2$ for $q=1$, and $\bar{\omega}_{\text {LSO }}=0.16$ for $q=6$. We obtained $\Delta \phi=0.62 \mathrm{rad}$ for $q=1$ and $\Delta \phi=2.66 \mathrm{rad}$ for $q=6$. This result shows that the difference between the $\mathrm{BB}$ and EOBNRlog $A$ functions entails, when considered by itself, a corresponding difference in the phasing (up to the LSO) that can be as large as $\sim 3$ rad depending on the mass ratio considered. However, the model of Ref. [29], that is based on the $A^{\mathrm{BB}}$ function, succeeded (like our EOBNRlog model) in getting an agreement with the NR waveform at the level of a $\%$ of a radian. This means that the $A$-dependent intrinsic difference in the (adiabatic) phasing that we are pointing out here can be (and has been) effectively compensated by other adjustable elements entering the model of Ref. [29] (notably parameters entering the radiation reaction, such as the argument of the $\rho_{\ell m}$ 's, the number of multipoles in the flux, a different NQC basis, the tuning of $\rho_{22}^{(4)}(\nu)$, etc.).

The conclusions of this comparative analysis of various EOB $A(u)$ functions are two sided. On the one hand, if we insist on trying to determine the $A$ function with the utmost accuracy needed to stay within an all-purposes equivalence class of $A$ functions, our results above show that the introduction of logarithmic contributions in the $A$ function cannot be reabsorbed by tuning log-less versions of the $\operatorname{EOB} A$ potential. As we know, from analytical PN work, that these logarithmic contributions do exist, we conclude that it is necessary to include them, and therefore to prefer the type of improved EOB model presented in this work to previous log-less versions of the EOB Hamiltonian. On the other hand, if we are ready to neglect the need of reaching an ideal all-purposes accuracy in the determination of the $A$ function, the overall conclusion of the comparison done in Fig. 18 is that accurate NR data (here the Caltech-Cornell-CITA ones) do constrain so much the value of the $\operatorname{EOB} A(u)$ potential (at least up to $u \sim 0.5$ ) that various ways of parametrizing the shape of the $A(u)$ potentials lead to final results that are rather close to each other. This comforts us in showing how the EOB formalism is able to extract from NR data reliable information about the strong-field dynamics of binary black holes.

\section{EXTENSION OF THE MODEL BY ANALYTIC CONTINUATION IN $\nu$}

In the present work, we have used a discrete sample of numerical simulations to complete an EOB model, notably through the use of suitable, NR-fitted NQC corrections. In order to be able to compute the predictions of such a NR-completed EOB model for arbitrary values of $\nu$, we need to fix a procedure for computing the six NQC parameters, $\left(a_{i}^{\ell m}(\nu), b_{i}^{\ell m}(\nu)\right)$, as continuous functions of $\nu$. [The remaining defining elements of the EOB model, notably $a_{5}^{c}$ and $a_{6}^{c}$ were already given as functions of $\nu$.]

One can think of two different ways of continuously extending the definition of the present EOB model to any value of $\nu$ : first, one can interpolate the discrete sample of $\left(a_{i}^{\ell m}, b_{i}^{\ell m}\right)$ values of the NQC parameters that we obtained (from the five numerical simulations with $q=$ $1,2,3,4,6)$ by fitting them to, say, quadratic polynomials in $\nu$; second, one can instead fit the original NR-extracted numerical values of $\left(A_{\ell m}, \dot{A}_{\ell m}, \ddot{A}_{\ell m}, \omega_{\ell m}, \dot{\omega}_{\ell m}, \ddot{\omega}_{\ell m}\right)$ to quadratic polynomials in $\nu$, and then, for any given $\nu$, determine $\left(a_{i}^{\ell m}(\nu), b_{i}^{\ell m}(\nu)\right)$ with the iterative procedure described above. We have explored in detail both procedures. The first one, i.e fitting the end parameters $\left(a_{i}^{\ell m}, b_{i}^{\ell m}\right)$ needed to compute an EOB waveform (and explicitly given in Table $\mathrm{V}$ for all $q$ 's) is clearly a faster 
TABLE IX. Fits of Zerilli-normalized multipolar quantities (amplitude, frequencies and derivatives) extracted at $t_{\text {extr }}^{\mathrm{NR}}(\nu)$ as function of $\nu$. Each quantity is fitted to a quadratic polynomial of the form $f_{\ell m}(\nu)=c_{2}^{\ell m} \nu^{2}+c_{1}^{\ell m} \nu+c_{0}^{\ell m}$. For the amplitude and its derivatives the full leading-order $\nu$-dependence $\left(\nu c_{\ell+\epsilon}(\nu)\right.$, see Eq. (22)) is factorized before fitting.

\begin{tabular}{|c|c|c|c|c|}
\hline & $\ell m$ & $c_{2}^{\ell m}$ & $c_{1}^{\ell m}$ & $c_{0}^{\ell m}$ \\
\hline & \begin{tabular}{ll|}
2 & 1
\end{tabular} & $1.8020 \times 10^{-1}$ & $-5.3482 \times 10^{-2}$ & $9.4465 \times 10^{-2}$ \\
\hline$A_{\ell m}$ & 2 & $3.6836 \times 10^{-1}$ & $2.3213 \times 10^{-2}$ & $2.9281 \times 10^{-1}$ \\
\hline \multirow[t]{2}{*}{$\overline{\nu c_{\ell+\epsilon}(\nu)}$} & 32 & $2.3484 \times 10^{-1}$ & $-5.1891 \times 10^{-2}$ & $1.5969 \times 10^{-2}$ \\
\hline & $\begin{array}{ll}3 & 3 \\
\end{array}$ & $1.5774 \times 10^{-1}$ & $7.1170 \times 10^{-3}$ & $5.1385 \times 10^{-2}$ \\
\hline \multirow[b]{2}{*}{$\dot{A}_{\ell m}$} & \begin{tabular}{ll|}
2 & 1
\end{tabular} & $1.3075 \times 10^{-2}$ & $-5.3660 \times 10^{-3}$ & $2.7088 \times 10^{-3}$ \\
\hline & 22 & $6.2259 \times 10^{-3}$ & $2.8059 \times 10^{-3}$ & $-1.5658 \times 10^{-3}$ \\
\hline \multirow[t]{2}{*}{$\nu c_{\ell+\epsilon}(\nu)$} & 32 & $2.7001 \times 10^{-2}$ & $-6.8708 \times 10^{-3}$ & $5.0927 \times 10^{-4}$ \\
\hline & $\begin{array}{ll}3 & 3 \\
\end{array}$ & $1.2320 \times 10^{-2}$ & $7.6133 \times 10^{-4}$ & $1.5238 \times 10^{-4}$ \\
\hline \multirow[b]{2}{*}{$\ddot{A}_{\ell m}$} & \begin{tabular}{ll|}
2 & 1
\end{tabular} & $5.2570 \times 10^{-4}$ & $-4.9124 \times 10^{-4}$ & $-1.1183 \times 10^{-4}$ \\
\hline & 22 & $1.4031 \times 10^{-3}$ & $-1.0071 \times 10^{-3}$ & $-7.4628 \times 10^{-4}$ \\
\hline \multirow[t]{2}{*}{$\nu c_{\ell+\epsilon}(\nu)$} & 32 & $4.9252 \times 10^{-3}$ & $-1.2516 \times 10^{-3}$ & $-3.1190 \times 10^{-6}$ \\
\hline & $\begin{array}{ll}3 & 3 \\
\end{array}$ & $-3.5470 \times 10^{-4}$ & $8.2613 \times 10^{-5}$ & $-1.3908 \times 10^{-4}$ \\
\hline \multirow{4}{*}{$\omega_{\ell m}$} & \begin{tabular}{ll|}
2 & 1
\end{tabular} & $-7.1306 \times 10^{-3}$ & $1.8015 \times 10^{-1}$ & $1.9488 \times 10^{-1}$ \\
\hline & 22 & $3.1848 \times 10^{-1}$ & $2.2996 \times 10^{-1}$ & $2.8788 \times 10^{-1}$ \\
\hline & 32 & -2.3137 & $5.3441 \times 10^{-1}$ & $3.5026 \times 10^{-1}$ \\
\hline & $\begin{array}{ll}3 & 3 \\
\end{array}$ & $3.7872 \times 10^{-1}$ & $4.1589 \times 10^{-1}$ & $4.4262 \times 10^{-1}$ \\
\hline \multirow{4}{*}{$\dot{\omega}_{\ell m}$} & \begin{tabular}{ll|}
2 & 1
\end{tabular} & $-5.4429 \times 10^{-2}$ & $2.3401 \times 10^{-2}$ & $8.6489 \times 10^{-3}$ \\
\hline & 22 & $2.6909 \times 10^{-2}$ & $1.3939 \times 10^{-2}$ & $6.3061 \times 10^{-3}$ \\
\hline & 32 & $-3.3131 \times 10^{-1}$ & $5.7770 \times 10^{-2}$ & $1.3219 \times 10^{-2}$ \\
\hline & 33 & $1.9620 \times 10^{-2}$ & $2.6984 \times 10^{-2}$ & $1.0610 \times 10^{-2}$ \\
\hline \multirow{4}{*}{$\ddot{\omega}_{\ell m}$} & 201 & $3.1509 \times 10^{-2}$ & $-5.7895 \times 10^{-3}$ & $9.1507 \times 10^{-4}$ \\
\hline & 22 & $2.2304 \times 10^{-3}$ & $3.2830 \times 10^{-4}$ & $9.6664 \times 10^{-5}$ \\
\hline & 32 & $-1.5297 \times 10^{-2}$ & $2.7862 \times 10^{-6}$ & $7.3264 \times 10^{-4}$ \\
\hline & $\begin{array}{ll}3 & 3\end{array}$ & $1.6612 \times 10^{-2}$ & $-2.0232 \times 10^{-3}$ & $3.0898 \times 10^{-4}$ \\
\hline
\end{tabular}

way to compute, for any $\nu$, a corresponding EOB waveform. Indeed, this approach does not require any iteration procedure.

We found that the fitted $\left(a_{i}^{\ell m}(\nu), b_{i}^{\ell m}(\nu)\right)$ 's give very accurate results for the multipoles we have at hand, i.e. $\ell=m=2, \ell=2, m=1, \ell=m=3$ and $\ell=3, m=2$. This allows us to construct EOB waveforms that are as accurate as the ones obtained by determining $\left(a_{i}^{\ell m}, b_{i}^{\ell m}\right)$ by the iterative procedure, discussed above, that uses the actual NR data. The coefficients of these quadratic fits are listed in Table $\mathrm{X}$.

By contrast, the determination of $\left(a_{i}^{\ell m}, b_{i}^{\ell m}\right)$ from quadratic fits of NR data (given in Table IX) is equally accurate for $\ell=m=2$ waveforms, but leads to slightly less accurate results for the subdominant multipoles. More precisely, this procedure introduces some visible, though small, differences between the EOB and NR waveform modulus around the peaks of the $(\ell=2, m=1$ and $\ell=m=3)$ waveforms. Note that, contrarily to the fits of the $\left(a_{i}, b_{i}\right)$ mentioned above (which relied only on the $q=1,2,3,4,6$ data), we have done quadratic fits of
TABLE X. Fits of the NQC parameters $\left(a_{i}^{\ell m}, b_{i}^{\ell m}\right)$ considered in this work as function of $\nu$. Each quantity is fitted to a quadratic polynomial of the form $f_{\ell m}(\nu)=c_{2}^{\ell m} \nu^{2}+c_{1}^{\ell m} \nu+c_{0}^{\ell m}$.

\begin{tabular}{|c|c|c|c|c|}
\hline & $\ell m$ & $c_{2}^{\ell m}$ & $c_{1}^{\ell m}$ & $c_{0}^{\ell m}$ \\
\hline \multirow{4}{*}{$a_{1}^{\ell m}(\nu)$} & 21 & 0.9150 & -0.6522 & 0.0340 \\
\hline & 22 & 2.1601 & -1.0937 & 0.0793 \\
\hline & $\begin{array}{ll}3 & 2\end{array}$ & 31.671 & -10.310 & 0.6844 \\
\hline & 33 & 2.6793 & -1.2792 & 0.1456 \\
\hline \multirow{4}{*}{$a_{2}^{\ell m}(\nu)$} & $\begin{array}{ll}2 & 1\end{array}$ & 1.9035 & 4.9785 & -0.7106 \\
\hline & 22 & -10.807 & 7.1420 & 0.7035 \\
\hline & $\begin{array}{ll}3 & 2\end{array}$ & -132.73 & 55.153 & -3.0449 \\
\hline & $\begin{array}{ll}3 & 3 \\
\end{array}$ & -12.932 & 10.634 & 0.4704 \\
\hline \multirow{4}{*}{$a_{3}^{\ell m}(\nu)$} & $\begin{array}{ll}2 & 1\end{array}$ & 2.6950 & -3.1603 & 0.8650 \\
\hline & 22 & -2.7666 & -0.1769 & 0.1012 \\
\hline & $\begin{array}{ll}3 & 2\end{array}$ & -19.734 & 1.8299 & 0.3031 \\
\hline & $\begin{array}{ll}3 & 3 \\
\end{array}$ & -0.8932 & -2.9229 & 0.5078 \\
\hline \multirow{4}{*}{$b_{1}^{\ell m}(\nu)$} & $\begin{array}{ll}2 & 1\end{array}$ & -2.2480 & 0.5304 & 0.2466 \\
\hline & 22 & -0.8568 & -0.2417 & 0.1929 \\
\hline & $\begin{array}{ll}3 & 2\end{array}$ & -1.3497 & -1.8083 & 0.5735 \\
\hline & $\begin{array}{ll}3 & 3 \\
\end{array}$ & -1.6468 & -0.1611 & 0.3464 \\
\hline \multirow{4}{*}{$b_{2}^{\ell m}(\nu)$} & $\begin{array}{ll}2 & 1\end{array}$ & 51.726 & -21.689 & 3.1616 \\
\hline & 22 & 9.6382 & -7.6453 & 0.3732 \\
\hline & $\begin{array}{ll}3 & 2\end{array}$ & -4.4860 & -7.7968 & 3.2538 \\
\hline & $\begin{array}{ll}3 & 3\end{array}$ & 6.9597 & -7.5958 & 0.0709 \\
\hline \multirow{4}{*}{$b_{3}^{\ell m}(\nu)$} & $\begin{array}{ll}2 & 1\end{array}$ & -112.81 & 30.559 & -0.0026 \\
\hline & 22 & -80.991 & 17.075 & -1.7974 \\
\hline & $\begin{array}{ll}3 & 2\end{array}$ & 3.2489 & -38.133 & 6.1648 \\
\hline & $\begin{array}{ll}3 & 3 \\
\end{array}$ & -213.46 & 52.819 & -3.4718 \\
\hline
\end{tabular}

$\left(A_{\ell m}, \dot{A}_{\ell m}, \ddot{A}_{\ell m}, \omega_{\ell m}, \dot{\omega}_{\ell m}, \ddot{\omega}_{\ell m}\right)$ to six numerical results, namely the Caltech-Cornell-CITA $q=(1,2,3,4,6)$ data together with the $q=\infty$ data of Ref. [42]. Given these fits, one then needs to solve for the NQC parameters. Actually, such a procedure is simplified by the fact that, as we said, the quadratic fits for the $a_{i}^{22}(\nu)$ 's (which are the only NQC parameters which need to be reinserted in the flux) can be used from the start, so that, contrary to the general case, one can get the needed values of the other NQC parameters in one go, without having to iterate the procedure.

Figure 19 illustrates the performances of the two different fitting procedures. The figure refers to mass ratio $q=2$ only (equivalent results are found for the other mass ratios, with improvements for larger values of $q$ ) and shows the following triple comparison for $\ell=m=2$ (top panel) and $\ell=2, m=1$ (medium panel), and $\ell=m=3$ (bottom panle) between: (i) the NR waveform frequency and modulus; (ii) the EOB waveform frequency and modulus obtained using the fits $\left(a_{i}^{\ell m}(\nu), b_{i}^{\ell m}(\nu)\right)$; (iii) the EOB waveform frequency and modulus obtained by fitting the numerical data extracted at $t_{\text {extr }}^{\mathrm{NR}}$, determining the NQC parameters in the usual way, but using the $a_{i}^{22}$ 

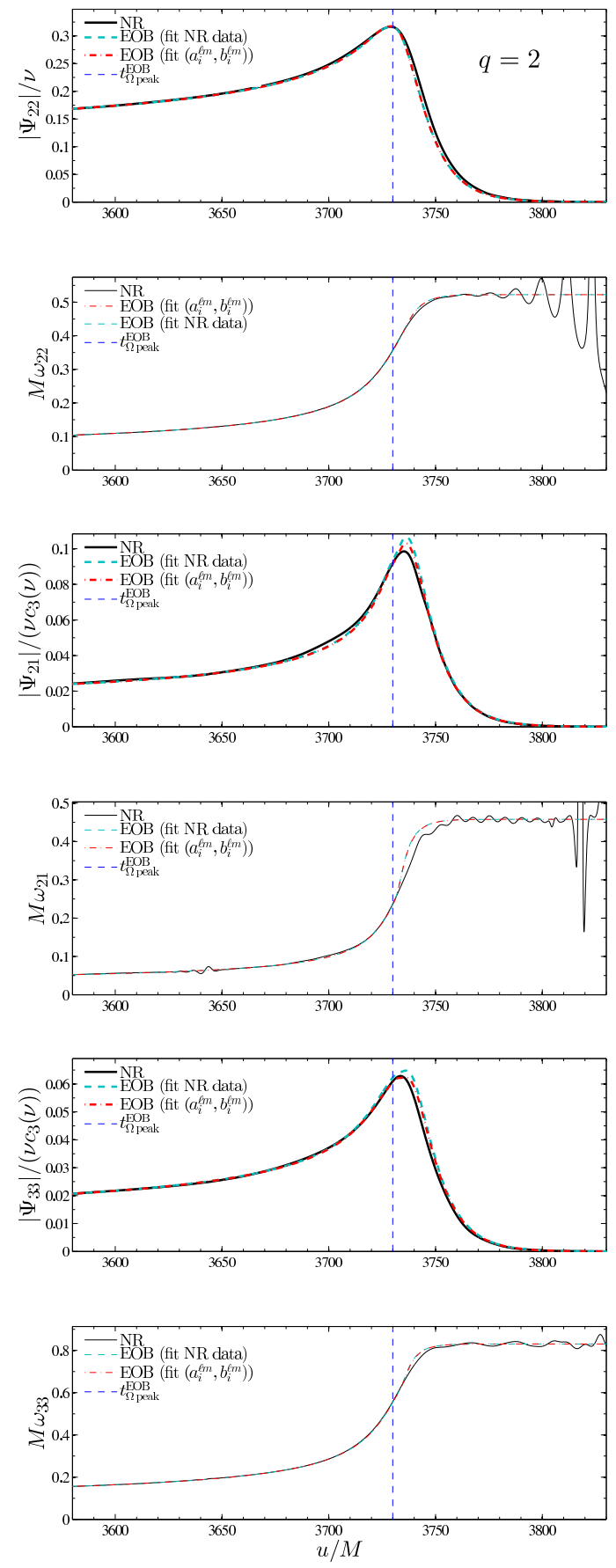

FIG. 19. (color online) Testing two possible fitting strategies to continuously extend the discrete sample of NQC parameters $\left(a_{i}^{\ell m}, b_{i}^{\ell m}\right)$ to any value of $\nu$ : comparison (for $q=2$ ) between $\ell=2$ and $\ell=3$ modulus and frequency.

fits of Table $\mathrm{X}$ to account for NQC corrections in the radiation reaction.

In conclusion, the prescription of using the $\left(a_{i}^{\ell m}(\nu), b_{i}^{\ell m}(\nu)\right)$ fits of Table $\mathrm{X}$ a priori looks as the best (and simplest) choice to obtain the NQC parameters interpolating between the discrete sample of
NR-computed $q$-values. Since the NR data we have at hand are limited to the $\ell=3$ multipole, we cannot check the reliability of the procedure also for higher values of $\ell$. We leave such an investigation to future work.

\section{CONCLUSIONS}

We have improved the EOB description of nonspinning coalescing black hole binaries by incorporating several recent analytical advances, namely:

(i) $4 \mathrm{PN}$ and $5 \mathrm{PN}$ logarithmic contributions to the conservative dynamics [35-38];

(ii) the $\mathcal{O}(\nu) 4 \mathrm{PN}$ nonlogarithmic contribution to the conservative dynamics [36, 38-40];

(iii) resummed horizon-absorption contributions to angular momentum loss [41, 42];

(iv) the radial component of the radiation reaction force implied by consistency with the azimuthal one [43];

(v) an additional 3.5PN contribution to the phase of the (factorized $[5,8,9]$ ) quadrupolar waveform [44].

Moreover, we have introduced new features in the EOB formalism, namely:

(a) a Padé resummation of the additional tail phases $\delta_{\ell m}$ of the factorized EOB waveform;

(b) a new way of matching the EOB waveform to the NR one by mapping the EOB time when the orbital frequency reaches a maximum $t_{\Omega \text { peak }}^{\mathrm{EOB}}$ to a specifically chosen ( $\nu$-dependent) NR time $t_{\text {extr }}^{\mathrm{NR}}(\nu)$ around merger, Eq. (55). More precisely, we impose [by using six next-to-quasi-circular (NQC) parameters] a $C^{2}$ contact between the amplitudes and the frequencies of the NR and $\mathrm{EOB}$ waveforms at the NR instant $t_{\mathrm{extr}}^{\mathrm{NR}}(\nu)$ which corresponds to $t_{\Omega \text { peak }}^{\mathrm{EOB}}$.

We have extracted new information from the NR data, namely:

(c) We showed how to extract from NR (curvature) phasing data the function $Q_{\omega}^{\mathrm{NR}}(\omega) \equiv \omega^{2} / \dot{\omega}$ which is an intrinsic measure of the phase evolution. We have given an explicit representation of the function $Q_{\omega}^{\mathrm{NR}}(\omega ; q)$, for $q=(1,2,3,4,6)$, in terms of some fitting coefficients (see Eqs. (61),(64) and Table III).

(d) We extracted data on the NR amplitude and frequency, together with their first two derivatives, at the specific $\nu$-dependent NR time $t_{\text {extr }}^{\mathrm{NR}}(\nu)$, which is located a little bit after the maximum of the quadrupolar waveform amplitude. We gave fitting formulas for the $\nu$-dependence of those quantities for several multipoles, see Table IX. 
Using such nonperturbative information from NR data, we showed how to complete the EOB model by:

1. Constraining the value of the main EOB radial potential, i.e. the $A(u ; \nu)$ function; and

2. Determining the coefficients entering the NQC correction factor Eq. (27).

Among these results, we think that the new expression of the NR-tuned $A$ function, containing logarithms, is more refined and more accurate than its previous determinations $[22,28,29]$. Let us recall that, as in previous work, the $A$ function is parametrized in terms of coefficients, here called $\left(a_{5}^{c}, a_{6}^{c}\right)$, entering a certain Padé approximant, $A^{\text {Pade }}\left(u ; \nu ; a_{5}^{c}, a_{6}^{c}\right)$, Eq. (10). Then NR data were used to constrain these parameters. We have delineated the reason why the two parameters $\left(a_{5}^{c}, a_{6}^{c}\right)$ entering the Padé definition of $A^{\text {Pade }}\left(u ; \nu ; a_{5}^{c}, a_{6}^{c}\right)$ are degenerate by giving a definition of equivalence classes of the pairs $\left(a_{5}^{c}, a_{6}^{c}\right)$ in terms of some $L_{\infty}$ norm of the $A(u)$ function. We have determined a good NR-tuned $A$ function by assuming a fixed value of $a_{5}^{c}\left(a_{5}^{c}=23.5\right.$ as suggested by recent GSF results $[38,48])$, and by then tuning the remaining parameter $a_{6}^{c}(\nu)$. We found that $a_{6}^{c}(\nu)$ can be simply represented by the mostly-linear function of $\nu$

$$
a_{6}^{c}(\nu)=[-110.5+147(1-4 \nu)]\left(1-\frac{1.5 \times 10^{-5}}{(0.26-\nu)^{2}}\right)^{1 / 2}
$$

where the last, nonlinear ${ }^{14}$ factor is relevant only in the range $0 . \overline{2} \lesssim \nu \leq 0.25$ (i.e., $1 \leq q \lesssim 2$ ). We think that the resulting function of $u$ and $\nu, \widetilde{A}^{\mathrm{EOBNR}}(u ; \nu) \equiv$ $A^{\text {Pade }}\left(u ; \nu ; 23.5, a_{6}^{c}(\nu)\right)$, yields an accurate representation of the $A(u ; \nu)$ function itself, independently of the way it was obtained. Moreover, we find remarkable that the good value of $A(u ; \nu)$ could be obtained already by considering only the inspiral phasing (before the LSO crossing) and was then checked to yield (together with the NR-determined NQC corrections) an excellent phasing agreement up to merger.

We have presented our improved EOB model in a self contained manner so as to allow interested readers to generate for themselves all our EOB results. We intend to make available soon a public version of our EOB codes. In view of the new physics that we have included in our EOB model, and of its excellent performance (obtained without introducing any ad hoc parameters) against the very accurate Caltech-Cornell-CITA data, we recommend to use this new EOB model (or small variations thereof) in future EOB works (in particular in extensions to spinning and/or tidally interacting systems).

14 Additional NR simulations in the mass-ratio range $1 \leq q \lesssim 2$ will be needed to probe/improve the nonlinear behavior of the $a_{6}^{c}(\nu)$ function there.

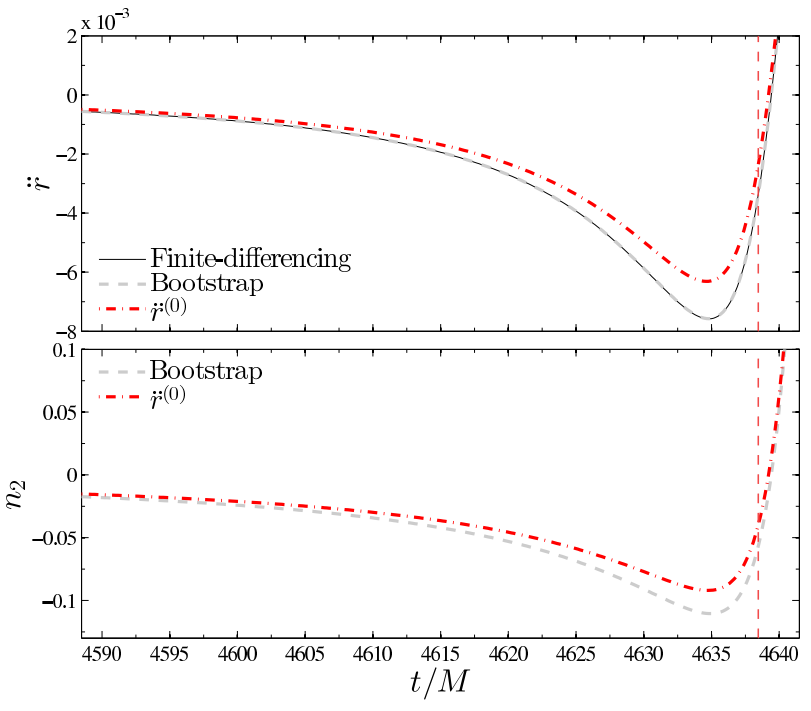

FIG. 20. (color online) Top: computation of $\ddot{r}$ with finite differencing and analytical iterations, and comparison with $(\ddot{r})^{(0)}$. Bottom: effect on the NQC basis vector $n_{2}$. The figure refers to $q=1$ with the choices $a_{5}^{c}=23.5$ and $a_{6}^{c}(0.25)=$ -101.876 . See text for discussion.

\section{ACKNOWLEDGMENTS}

We are grateful L. Buchman, L. Kidder, H. Pfeiffer, M. Scheel, and B. Szilágyi for making available to us the most recent and accurate simulations of the CaltechCornell-CITA collaboration. We are particularly indebted to H. Pfeiffer for continuous help and informative communications about the waveforms, and to L. Buchman for clarifications about the waveform error budget. We thank E. Barausse, A. Buonanno, Y. Pan and A. Taracchini for informative communications about the implementation of their EOB model, Ref. [28, 29], as well as $\mathrm{G}$. Faye for checking the 3.5PN term of the $\delta_{22}$ additional phase, Eq. (24). S.B. was supported in part by DFG grant SFB/Transregio 7 "Gravitational Wave Astronomy". S.B. thanks IHES for hospitality during the development of part of this work.

\section{Appendix A: On the computation of $\ddot{r}$}

In the definition of the NQC correction $n_{2}$, Eq. (28b), we used for the second time derivative of the relative separation $r$ the quantity $(\ddot{r})^{(0)}$, which is the value of $\ddot{r}$ along the conservative dynamics, i.e. neglecting the contributions proportional to $\mathcal{F}$. This choice is made for efficiency's sake because it is faster to compute $(\ddot{r})^{(0)}$ along the dynamics. In spite of the neglect of $\mathcal{F}$ in its computation, $(\ddot{r})^{(0)}$ does represent an allowed NQC correction because it vanishes (together with $\dot{r}$ and the exact value of $\ddot{r}$ ) in the circular limit (see below).

For completeness, let us discuss here how to compute a more exact value of $\ddot{r}$ along the dynamics and how the 
TABLE XI. Fits of Zerilli-normalized multipolar quantities of the numerical waveforms (modulus, frequency and their derivatives) measured at the peak of each multipole ("maxima") as function of $\nu$. Each quantity is fitted to a quadratic polynomial of the form $f_{\ell m}(\nu)=c_{2}^{\ell m} \nu^{2}+c_{1}^{\ell m} \nu+c_{0}^{\ell m}$. For the modulus and its derivatives the leading order $\nu$-dependence (Eq. 22) is factorized before fitting.

\begin{tabular}{|c|c|c|c|c|}
\hline & $\ell m$ & $c_{2}^{\ell m}$ & $c_{1}^{\ell m}$ & $c_{0}^{\ell m}$ \\
\hline & $2 \quad 1$ & $2.9410 \times 10^{-1}$ & $-1.0286 \times 10^{-1}$ & $1.0691 \times 10^{-1}$ \\
\hline$A_{\ell m}$ & 22 & $3.8132 \times 10^{-1}$ & $1.3011 \times 10^{-2}$ & $2.9467 \times 10^{-1}$ \\
\hline \multirow[t]{2}{*}{$\overline{\nu c_{\ell+\epsilon}(\nu)}$} & 32 & $3.9814 \times 10^{-1}$ & $-9.2149 \times 10^{-2}$ & $1.8310 \times 10^{-2}$ \\
\hline & 33 & $2.0896 \times 10^{-1}$ & $4.7198 \times 10^{-3}$ & $5.1463 \times 10^{-2}$ \\
\hline \multirow[b]{2}{*}{$\ddot{A}_{\ell m}$} & 25 & $-5.2646 \times 10^{-3}$ & $6.1932 \times 10^{-4}$ & $-5.4059 \times 10^{-4}$ \\
\hline & 22 & $1.5609 \times 10^{-4}$ & $-1.4628 \times 10^{-3}$ & $-4.8017 \times 10^{-4}$ \\
\hline \multirow[t]{2}{*}{$\overline{\nu c_{\ell+\epsilon}(\nu)}$} & $\begin{array}{ll}3 & 2\end{array}$ & $1.8500 \times 10^{-4}$ & $2.2093 \times 10^{-4}$ & $-1.4184 \times 10^{-4}$ \\
\hline & 33 & $-3.4677 \times 10^{-3}$ & $-6.6072 \times 10^{-6}$ & $-1.6713 \times 10^{-4}$ \\
\hline \multirow{4}{*}{$\omega_{\ell m}$} & $2 \quad 1$ & $5.8728 \times 10^{-1}$ & $-8.3459 \times 10^{-2}$ & $2.9074 \times 10^{-1}$ \\
\hline & 22 & $4.1410 \times 10^{-1}$ & $2.4377 \times 10^{-1}$ & $2.7221 \times 10^{-1}$ \\
\hline & 32 & 3.6315 & $-9.5776 \times 10^{-1}$ & $4.5459 \times 10^{-1}$ \\
\hline & 33 & 1.0192 & $5.4557 \times 10^{-1}$ & $4.5319 \times 10^{-1}$ \\
\hline \multirow{4}{*}{$\dot{\omega}_{\ell m}$} & $2 \quad 1$ & $-2.2041 \times 10^{-1}$ & $1.0228 \times 10^{-1}$ & $6.2835 \times 10^{-4}$ \\
\hline & 22 & $2.8060 \times 10^{-2}$ & $1.4581 \times 10^{-2}$ & $5.8725 \times 10^{-3}$ \\
\hline & 32 & $-2.8225 \times 10^{-1}$ & $3.7702 \times 10^{-2}$ & $1.6036 \times 10^{-2}$ \\
\hline & $\begin{array}{ll}3 & 3 \\
\end{array}$ & $2.5253 \times 10^{-2}$ & $2.7690 \times 10^{-2}$ & $1.0871 \times 10^{-2}$ \\
\hline \multirow{4}{*}{$\ddot{\omega}_{\ell m}$} & 21 & $7.8607 \times 10^{-3}$ & $1.5684 \times 10^{-2}$ & $-3.5511 \times 10^{-3}$ \\
\hline & 22 & $2.3604 \times 10^{-3}$ & $7.2810 \times 10^{-5}$ & $2.2436 \times 10^{-4}$ \\
\hline & 32 & $-8.7028 \times 10^{-2}$ & $1.7233 \times 10^{-2}$ & $8.6570 \times 10^{-5}$ \\
\hline & & $-1.1065 \times 10^{-2}$ & $-2.3899 \times 10^{-6}$ & $2.1351 \times 10^{-4}$ \\
\hline
\end{tabular}

result differs from $(\ddot{r})^{(0)}$. Let us first recall that along the EOB equations of motion $\dot{r}$ is, at any moment, a function of the phase space variables: $\dot{r}=\dot{r}\left(r(t), p_{\varphi}(t), p_{r_{*}}(t)\right)$. Therefore, its total time derivative is the sum of three partial contributions

$$
\ddot{r}=\frac{\partial \dot{r}}{\partial r} \dot{r}+\frac{\partial \dot{r}}{\partial p_{r_{*}}} \dot{p}_{r_{*}}+\frac{\partial \dot{r}}{\partial p_{\varphi}} \dot{p}_{\varphi} .
$$

Using the other EOB equations of motion, this equation reads explicitly

$$
\ddot{r}=\frac{\partial \dot{r}}{\partial r} \dot{r}+\frac{\partial \dot{r}}{\partial p_{r_{*}}}\left(\hat{\mathcal{F}}_{r_{*}}-\frac{\partial \hat{H}_{\mathrm{EOB}}}{\partial r_{*}}\right)+\frac{\partial \dot{r}}{\partial p_{\varphi}} \hat{\mathcal{F}}_{\varphi}
$$

where $\partial H_{\mathrm{EOB}} / \partial r_{*} \equiv(A / B)^{1 / 2} \partial H_{\mathrm{EOB}} / \partial r$.

By definition, the circular dynamics limit corresponds to setting $\dot{r}=0=p_{r_{*}}$ and $\partial H_{\mathrm{EOB}} / \partial r=0$. One then sees that, along the circular dynamics, one has also $\mathcal{F}_{r_{*}} \propto$ $p_{r_{*}}=0$, and (using $\left.\dot{r}=C\left(r, p_{r_{*}}, p_{\varphi}\right) p_{r_{*}}\right) \partial \dot{r} / \partial p_{\varphi} \propto p_{r_{*}}=$ 0 . As a consequence, both $\ddot{r}$ and $(\ddot{r})^{(0)}$, defined by setting to zero the contributions proportional to $\mathcal{F}$, i.e.

$$
(\ddot{r})^{(0)}=\frac{\partial \dot{r}}{\partial r} \dot{r}-\frac{\partial \dot{r}}{\partial p_{r_{*}}} \frac{\partial \hat{H}_{\mathrm{EOB}}}{\partial r_{*}},
$$

vanish in the circular dynamics approximation. This shows that we can use either the exact $\ddot{r}$ or its "geodesic" approximation $(\ddot{r})^{(0)}$ to define the second element of the "NQC basis", $n_{2}=\ddot{r} /\left(r \Omega^{2}\right)$.

When using the definition $n_{2}=(\ddot{r})^{(0)} /\left(r \Omega^{2}\right)$, Eq. (A3) allows one to compute immediately $n_{2}$ along the exact dynamics. By contrast, if one wished to use the definition $n_{2}^{\prime}=\ddot{r} /\left(r \Omega^{2}\right)$, a complication arises. Indeed, as contributions proportional to $\hat{\mathcal{F}}_{r_{*}}$ and $\hat{\mathcal{F}}_{\varphi}$ appear on the r.h.s. of Eq. (A2), and as these contain the squared modulus of the NQC factor (i.e., for each multipole, a factor $\left|1+\sum_{j} a_{j}^{\ell m} n_{j}\right|^{2}$ ) we see that $n_{2}^{\prime} \propto \ddot{r}$ now appears on both sides of Eq. (A2).

Schematically, defining $\xi=\left(r, p_{r_{*}}, p_{\varphi}\right)$, Eq. (A2) has the structure

$$
\ddot{r}=a(\xi)+b(\xi) \hat{\mathcal{F}}_{r_{*}}(\xi, \ddot{r})+c(\xi) \hat{\mathcal{F}}_{\varphi}(\xi, \ddot{r})
$$

which only gives an implicit equation for determining the exact $\ddot{r}$ along the dynamics. We can however get an explicit expression for $\ddot{r}$ by an iterative procedure. Inserting $\ddot{r}^{(0)}$ as lowest order approximation on the r.h.s. of Eq. (A4) defines an improved value, say $(\ddot{r})^{(1)}$ for $\ddot{r}$, namely

$$
\ddot{r}^{(1)}=a(\xi)+b(\xi) \hat{\mathcal{F}}_{r_{*}}\left(\xi, \ddot{r}^{(0)}\right)+c(\xi) \hat{\mathcal{F}}_{\varphi}\left(\xi, \ddot{r}^{(0)}\right) .
$$

By iterating the procedure once more, we then get

$$
\ddot{r}^{(2)}=a(\xi)+b(\xi) \hat{\mathcal{F}}_{r_{*}}\left(\xi, \ddot{r}^{(1)}\right)+c(\xi) \hat{\mathcal{F}}_{\varphi}\left(\xi, \ddot{r}^{(1)}\right) .
$$

The result (A6) leads to a sufficiently accurate computation of $\ddot{r}$ up to merger, as illustrated in the top panel of Fig. 20. However, the recursive presence of the flux in this iteration substantially increases (by approximately a factor 4) the computational time needed to produce an EOB waveform. This is why we prefer to use $n_{2}=\ddot{r}^{(0)} /\left(r \Omega^{2}\right)$. Anyway, as Fig. 20 shows, $n_{2}$ and $n_{2}^{\prime}$ are numerically quite similar. In view of the arguments above their differences are essentially absorbed in a redefinition of the coefficients $a_{i}$.

\section{Appendix B: NQC factor determined using NR data at $t_{A_{22} \text { peak }}^{\mathrm{NR}}$}

In the text, we argued that it was advantageous to determine NQC corrections by matching the EOB waveform (considered at $t_{\Omega \text { peak }}^{\mathrm{EOB}}$ ) to the NR waveform considered at the time $t_{\text {extr }}^{\mathrm{NR}}$. Let us illustrate here (see Fig. 21) in the case $q=6$ the slightly different (but significantly worsened) EOB waveform obtained when one instead matches the $\ell=m=2$ EOB waveform (considered at time $\left.t_{\Omega \text { peak }}^{\mathrm{EOB}}\right)$ to the NR waveform considered at the time $t_{A_{22} \text { peak }}^{\mathrm{NR}}$ (as was done in early EOB works). Figure 21 uses as before $6 \mathrm{NQC}$ corrections and the value $a_{6}^{c}(6 / 49)=-44.67$. However, the NR extraction point, which is also used as NQC determination point, is now $t_{A_{22} \text { peak }}^{\mathrm{NR}}$. 


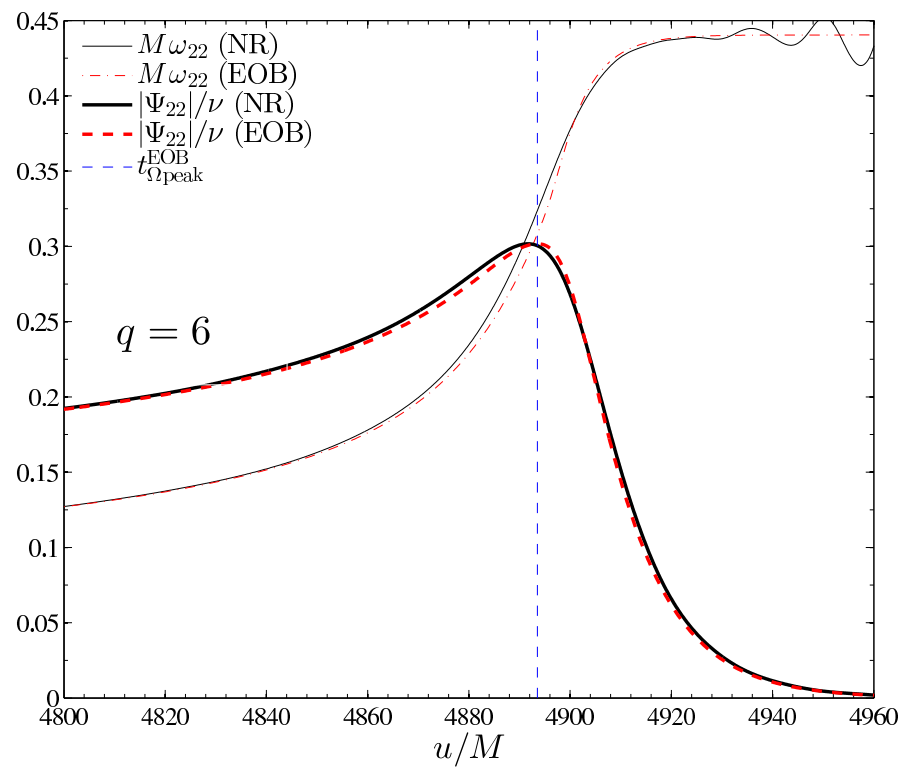

FIG. 21. (color online) Mass ratio $q=6$ : EOB waveform (frequency and modulus) obtained by determining NQC corrections from $\mathrm{NR}$ data extracted at $t_{A_{22} \text { peak }}^{\mathrm{NR}}$ instead of $t_{\mathrm{extr}}^{\mathrm{NR}}$.

The fits of the vector of NR quantities $\left(A_{\ell m}^{\mathrm{NR}}, \ddot{A}_{\ell m}^{\mathrm{NR}}, \omega_{\ell m}^{\mathrm{NR}}, \dot{\omega}_{\ell m}^{\mathrm{NR}}, \ddot{\omega}_{\ell m}^{\mathrm{NR}}\right)$ now measured at the location of the maximum of each multipole are given in Table XI and include, as before, the test-mass information. We checked that these fits are compatible with the fits given in Table II of Ref. [28].

When comparing Fig. 21 with the bottom left panel of Fig. 11, we see that, though the effect of having replaced $t_{\mathrm{extr}}^{\mathrm{NR}}$ by $t_{A_{22} \text { peak }}^{\mathrm{NR}}$ is small, it leads to visible differences. In particular, one sees that the frequency evolution near merger was more accurately captured in Fig. 11 than in Fig. 21.

\section{Appendix C: Effect of including NQC corrections to higher multipoles in the radiation reaction}

In this Appendix we explore the effect of including the NQC correction factor in the higher multipole contributions to radiation reaction, specifically in some of the main subdominant multipoles, $\hat{h}_{21}^{\mathrm{NQC}}, \hat{h}_{33}^{\mathrm{NQC}}$ and $\hat{h}_{32}^{\mathrm{NQC}}$. [By contrast in the main text we NQC corrected only $\hat{h}_{22}^{\mathrm{NQC}}$ in the radiation reaction]. Note that with our choice $x=v_{\varphi}^{2}$ of the argument in $\rho_{\ell m}(x)$ we need larger NQC modulus correction factors than Ref. [28] which used $x=\Omega^{2 / 3}$. Indeed as during the plunge $\Omega^{2 / 3}$ is larger than $v_{\varphi}^{2}$ and as the function $\rho_{\ell m}(x)$ is a decreasing function of its argument, one has, along the EOB dynam- ics, $\left(\rho_{\ell m}\left(v_{\varphi}^{2}\right)\right)^{\ell}>\left(\rho_{\ell m}\left(\Omega^{2 / 3}\right)\right)^{\ell}$. Therefore the inclusion of NQC corrections for higher multipoles is apriori more significant within our EOB setup than within the one of Ref. [28]. We focus on the mass ratio $q=6$ only, because

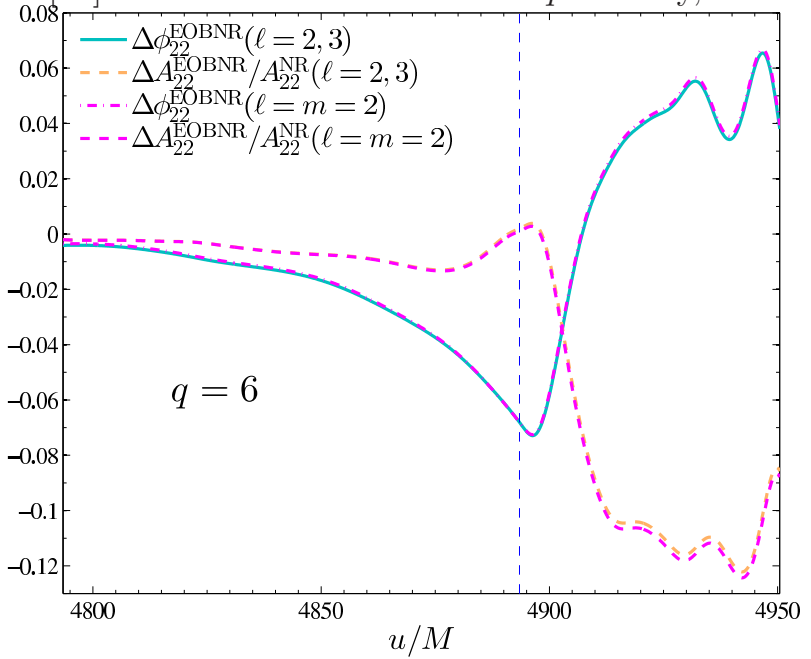

FIG. 22. (color online) Negligible effect on the phasing (and modulus), for $q=6$, of including $\ell=2, m=1$ and $\ell=3$, $(m=2,3)$ NQC corrections to the energy flux beyond the $\ell=m=2$ one.

subdominant multipoles do not significantly contribute when $q \sim 1$. Figure 22 compares the phase difference and the fractional amplitude difference for two EOB models: one with the standard $h_{22}$-only NQC flux correction (magenta online), and another one which includes in addition the three subleading NQC factors $\hat{h}_{21}^{\mathrm{NQC}}, \hat{h}_{33}^{\mathrm{NQC}}$ and $\hat{h}_{32}^{\mathrm{NQC}}$. The effect of this inclusion is totally negligible, so that it is justified to include only the $\ell=m=2 \mathrm{NQC}$ correction to the radiation reaction.

\section{Appendix D: Explicit expression of $\rho_{\ell m}(x)$ and $\delta_{\ell m}(y)$}

In this Appendix we list the explicit expressions of the residual amplitude, $\rho_{\ell m}(x)$, and phase, $\delta_{\ell m}(y)$ corrections that we have implemented in our EOB code. They rely on the results of Refs. [5, 51]. We give explicit expressions for all multipoles up to $\ell=8$ included. Such expressions are given at the $3^{+2} \mathrm{PN}$ approximation, i.e. the $3 \mathrm{PN}$-accurate, $\nu \neq 0$ results of Ref. [5] are $h y$ bridized with the $5 \mathrm{PN}$-accurate, $\nu=0$, terms obtained in Ref. [51]. Let us recall that we used here the following values of the arguments of these functions: $x \rightarrow v_{\varphi}^{2}$ in $\rho_{\ell m}(x)$ and $y \rightarrow\left(H_{\mathrm{EOB}} \Omega\right)^{2 / 3}$. 


$$
\begin{aligned}
& \rho_{22}(x ; \nu)=1+\left(\frac{55 \nu}{84}-\frac{43}{42}\right) x+\left(\frac{19583 \nu^{2}}{42336}-\frac{33025 \nu}{21168}-\frac{20555}{10584}\right) x^{2} \\
& +\left(\frac{10620745 \nu^{3}}{39118464}-\frac{6292061 \nu^{2}}{3259872}+\frac{41 \pi^{2} \nu}{192}-\frac{48993925 \nu}{9779616}-\frac{428}{105} \text { eulerlog }_{2}(x)+\frac{1556919113}{122245200}\right) x^{3} \\
& +\left(\frac{9202}{2205} \text { eulerlog }_{2}(x)-\frac{387216563023}{160190110080}\right) x^{4}+\left(\frac{439877}{55566} \text { eulerlog }_{2}(x)-\frac{16094530514677}{533967033600}\right) x^{5}, \\
& \rho_{21}(x ; \nu)=1+\left(\frac{23 \nu}{84}-\frac{59}{56}\right) x+\left(\frac{617 \nu^{2}}{4704}-\frac{10993 \nu}{14112}-\frac{47009}{56448}\right) x^{2} \\
& +\left(\frac{7613184941}{2607897600}-\frac{107}{105} \text { eulerlog }_{1}(x)\right) x^{3}+\left(\frac{6313}{5880} \text { eulerlog }_{1}(x)-\frac{1168617463883}{911303737344}\right) x^{4} \\
& +\left(-\frac{63735873771463}{16569158860800}+\frac{5029963}{5927040} \text { eulerlog }_{1}(x)\right) x^{5}, \\
& \rho_{33}(x ; \nu)=1+\left(\frac{2 \nu}{3}-\frac{7}{6}\right) x+\left(\frac{149 \nu^{2}}{330}-\frac{1861 \nu}{990}-\frac{6719}{3960}\right) x^{2}+\left(\frac{3203101567}{227026800}-\frac{26}{7} \operatorname{eulerlog}_{3}(x)\right) x^{3} \\
& +\left(\frac{13}{3} \text { eulerlog }_{3}(x)-\frac{57566572157}{8562153600}\right) x^{4}+\left(-\frac{903823148417327}{30566888352000}+\frac{87347}{13860} \text { eulerlog }_{3}(x)\right) x^{5}, \\
& \rho_{32}(x ; \nu)=1+\frac{320 \nu^{2}-1115 \nu+328}{270(3 \nu-1)} x+\frac{3085640 \nu^{4}-20338960 \nu^{3}-4725605 \nu^{2}+8050045 \nu-1444528}{1603800(1-3 \nu)^{2}} x^{2} \\
& +\left(\frac{5849948554}{940355325}-\frac{104}{63} \text { eulerlog }_{2}(x)\right) x^{3}+\left(-\frac{10607269449358}{3072140846775}+\frac{17056}{8505} \text { eulerlog }_{2}(x)\right) x^{4}, \\
& \rho_{31}(x ; \nu)=1+\left(-\frac{2 \nu}{9}-\frac{13}{18}\right) x+\left(-\frac{829 \nu^{2}}{1782}-\frac{1685 \nu}{1782}+\frac{101}{7128}\right) x^{2}+\left(\frac{11706720301}{6129723600}-\frac{26}{63} \text { eulerlog } \log _{1}(x)\right) x^{3}
\end{aligned}
$$

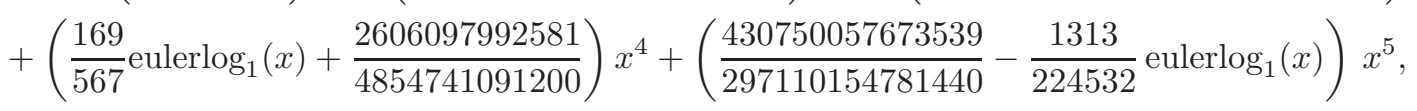

$$
\begin{aligned}
& \rho_{44}(x ; \nu)=1+\frac{2625 \nu^{2}-5870 \nu+1614}{1320(3 \nu-1)} x \\
& +\frac{1252563795 \nu^{4}-6733146000 \nu^{3}-313857376 \nu^{2}+2338945704 \nu-511573572}{317116800(1-3 \nu)^{2}} x^{2} \\
& +\left(\frac{16600939332793}{1098809712000}-\frac{12568}{3465} \text { eulerlog }_{4}(x)\right) x^{3}+\left(\frac{845198}{190575} \text { eulerlog }_{4}(x)-\frac{172066910136202271}{19426955708160000}\right) x^{4}, \\
& \rho_{43}(x ; \nu)=1+\frac{160 \nu^{2}-547 \nu+222}{176(2 \nu-1)} x-\frac{6894273}{7047040} x^{2}+\left(-\frac{1571}{770} \text { eulerlog } \log _{3}(x)+\frac{1664224207351}{195343948800}\right) x^{3} \\
& +\left(-\frac{2465107182496333}{460490801971200}+\frac{174381}{67760} \text { eulerlog }_{3}(x)\right) x^{4}, \\
& \rho_{42}(x ; \nu)=1+\frac{285 \nu^{2}-3530 \nu+1146}{1320(3 \nu-1)} x \\
& +\frac{-379526805 \nu^{4}-3047981160 \nu^{3}+1204388696 \nu^{2}+295834536 \nu-114859044}{317116800(1-3 \nu)^{2}} x^{2} \\
& +\left(\frac{848238724511}{219761942400}-\frac{3142}{3465} \text { eulerlog }_{2}(x)\right) x^{3}+\left(\frac{300061}{381150} \text { eulerlog }_{2}(x)-\frac{12864377174485679}{19426955708160000}\right) x^{4},
\end{aligned}
$$




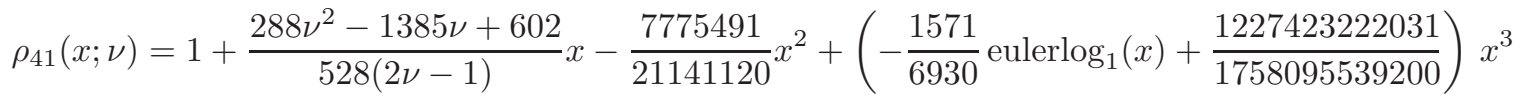

$$
\begin{aligned}
& +\left(-\frac{29584392078751453}{37299754959667200}+\frac{67553}{261360} \text { eulerlog }_{1}(x)\right) x^{4} \\
& \rho_{55}(x ; \nu)=1+\frac{512 \nu^{2}-1298 \nu+487}{390(2 \nu-1)} x-\frac{3353747}{2129400} x^{2}+\left(-\frac{1546}{429} \text { eulerlog }_{5}(x)+\frac{190606537999247}{11957879934000}\right) x^{3} \\
& +\left(-\frac{1213641959949291437}{118143853747920000}+\frac{376451}{83655} \text { eulerlog }_{5}(x)\right) x^{4}, \\
& \rho_{54}(x ; \nu)=1+\frac{33320 \nu^{3}-127610 \nu^{2}+96019 \nu-17448}{13650\left(5 \nu^{2}-5 \nu+1\right)} x-\frac{16213384}{15526875} x^{2} \\
& +\left(-\frac{24736}{10725} \text { eulerlog }_{4}(x)+\frac{6704294638171892}{653946558890625}\right) x^{3} \\
& \rho_{53}(x ; \nu)=1+\frac{176 \nu^{2}-850 \nu+375}{390(2 \nu-1)} x-\frac{410833}{709800} x^{2}+\left(-\frac{4638}{3575} \operatorname{eulerlog}_{3}(x)+\frac{7618462680967}{1328653326000}\right) x^{3} \\
& +\left(-\frac{77082121019870543}{39381284582640000}+\frac{2319}{1859} \text { eulerlog }_{3}(x)\right) x^{4} \\
& \rho_{52}(x ; \nu)=1+\frac{21980 \nu^{3}-104930 \nu^{2}+84679 \nu-15828}{13650\left(5 \nu^{2}-5 \nu+1\right)} x-\frac{7187914}{15526875} x^{2} \\
& +\left(\frac{1539689950126502}{653946558890625}-\frac{6184}{10725} \text { euler } \log _{2}(x)\right) x^{3} \\
& \rho_{51}(x ; \nu)=1+\frac{8 \nu^{2}-626 \nu+319}{390(2 \nu-1)} x-\frac{31877}{304200} x^{2}+\left(-\frac{1546}{10725} \text { eulerlog }_{1}(x)+\frac{7685351978519}{11957879934000}\right) x^{3} \\
& +\left(-\frac{821807362819271}{10740350340720000}+\frac{22417}{190125} \text { eulerlog }_{1}(x)\right) x^{4} \\
& \rho_{66}(x ; \nu)=1+\frac{273 \nu^{3}-861 \nu^{2}+602 \nu-106}{84\left(5 \nu^{2}-5 \nu+1\right)} x-\frac{1025435}{659736} x^{2}+\left(-\frac{3604}{1001} \text { eulerlog } \log _{6}(x)+\frac{610931247213169}{36701493028200}\right) x^{3}
\end{aligned}
$$$$
\rho_{65}(x ; \nu)=1+\frac{220 \nu^{3}-910 \nu^{2}+838 \nu-185}{144\left(3 \nu^{2}-4 \nu+1\right)} x-\frac{59574065}{54286848} x^{2}+\left(-\frac{22525}{9009} \text { eulerlog }_{5}(x)+\frac{67397117912549267}{5798416452820992}\right) x^{3}
$$

$$
\rho_{64}(x ; \nu)=1+\frac{133 \nu^{3}-581 \nu^{2}+462 \nu-86}{84\left(5 \nu^{2}-5 \nu+1\right)} x-\frac{476887}{659736} x^{2}+\left(-\frac{14416}{9009} \text { eulerlog }_{4}(x)+\frac{180067034480351}{24467662018800}\right) x^{3}
$$$$
\rho_{63}(x ; \nu)=1+\frac{156 \nu^{3}-750 \nu^{2}+742 \nu-169}{144\left(3 \nu^{2}-4 \nu+1\right)} x-\frac{152153941}{271434240} x^{2}+\left(-\frac{901}{1001} \text { eulerlog }_{3}(x)+\frac{116042497264681103}{28992082264104960}\right) x^{3}
$$

$\rho_{62}(x ; \nu)=1+\frac{49 \nu^{3}-413 \nu^{2}+378 \nu-74}{84\left(5 \nu^{2}-5 \nu+1\right)} x-\frac{817991}{3298680} x^{2}+\left(-\frac{3604}{9009}\right.$ eulerlog $\left._{2}(x)+\frac{812992177581}{453104852200}\right) x^{3}$

$\rho_{61}(x ; \nu)=1+\frac{124 \nu^{3}-670 \nu^{2}+694 \nu-161}{144\left(3 \nu^{2}-4 \nu+1\right)} x-\frac{79192261}{271434240} x^{2}+\left(-\frac{901}{9009}\right.$ eulerlog $\left.\log _{1}(x)+\frac{6277796663889319}{28992082264104960}\right) x^{3}$ 
$\rho_{77}(x ; \nu)=1+\frac{1380 \nu^{3}-4963 \nu^{2}+4246 \nu-906}{714\left(3 \nu^{2}-4 \nu+1\right)} x-\frac{32358125}{20986602} x^{2}+\left(-\frac{11948}{3315} \operatorname{eulerlog}_{7}(x)+\frac{66555794049401803}{3856993267327200}\right) x^{3}$

$\rho_{76}(x ; \nu)=1+\frac{6104 \nu^{4}-29351 \nu^{3}+37828 \nu^{2}-16185 \nu+2144}{1666\left(7 \nu^{3}-14 \nu^{2}+7 \nu-1\right)} x-\frac{195441224}{171390583} x^{2}$,

$\rho_{75}(x ; \nu)=1+\frac{804 \nu^{3}-3523 \nu^{2}+3382 \nu-762}{714\left(3 \nu^{2}-4 \nu+1\right)} x-\frac{17354227}{20986602} x^{2}+\left(-\frac{59740}{32487} \operatorname{eulerlog}_{5}(x)+\frac{192862646381533}{22039961527584}\right) x^{3}$,

$\rho_{74}(x ; \nu)=1+\frac{41076 \nu^{4}-217959 \nu^{3}+298872 \nu^{2}-131805 \nu+17756}{14994\left(7 \nu^{3}-14 \nu^{2}+7 \nu-1\right)} x-\frac{2995755988}{4627545741} x^{2}$

$\rho_{73}(x ; \nu)=1+\frac{420 \nu^{3}-2563 \nu^{2}+2806 \nu-666}{714\left(3 \nu^{2}-4 \nu+1\right)} x-\frac{7804375}{20986602} x^{2}+\left(-\frac{35844}{54145} \operatorname{eulerlog}_{3}(x)+\frac{1321461327981547}{428554807480800}\right) x^{3}$

$\rho_{72}(x ; \nu)=1+\frac{32760 \nu^{4}-190239 \nu^{3}+273924 \nu^{2}-123489 \nu+16832}{14994\left(7 \nu^{3}-14 \nu^{2}+7 \nu-1\right)} x-\frac{1625746984}{4627545741} x^{2}$

$\rho_{71}(x ; \nu)=1+\frac{228 \nu^{3}-2083 \nu^{2}+2518 \nu-618}{714\left(3 \nu^{2}-4 \nu+1\right)} x-\frac{1055091}{6995534} x^{2}+\left(-\frac{11948}{162435}\right.$ eulerlog $\left._{1}(x)+\frac{142228318411021}{550999038189600}\right) x^{3}$

$\rho_{88}(x ; \nu)=1+\frac{12243 \nu^{4}-53445 \nu^{3}+64659 \nu^{2}-26778 \nu+3482}{2736\left(7 \nu^{3}-14 \nu^{2}+7 \nu-1\right)} x-1.5337092502821381 x^{2}$,

$\rho_{87}(x ; \nu)=1+\frac{38920 \nu^{4}-207550 \nu^{3}+309498 \nu^{2}-154099 \nu+23478}{18240\left(4 \nu^{3}-10 \nu^{2}+6 \nu-1\right)} x-1.175404252991305 x^{2}$

$\rho_{86}(x ; \nu)=1+\frac{2653 \nu^{4}-13055 \nu^{3}+17269 \nu^{2}-7498 \nu+1002}{912\left(7 \nu^{3}-14 \nu^{2}+7 \nu-1\right)} x-0.9061610303170207 x^{2}$

$\rho_{85}(x ; \nu)=1+\frac{6056 \nu^{4}-34598 \nu^{3}+54642 \nu^{2}-28055 \nu+4350}{3648\left(4 \nu^{3}-10 \nu^{2}+6 \nu-1\right)} x-0.7220789990670207 x^{2}$,

$\rho_{84}(x ; \nu)=1+\frac{4899 \nu^{4}-28965 \nu^{3}+42627 \nu^{2}-19434 \nu+2666}{2736\left(7 \nu^{3}-14 \nu^{2}+7 \nu-1\right)} x-0.47652059150068155 x^{2}$

$\rho_{83}(x ; \nu)=1+\frac{24520 \nu^{4}-149950 \nu^{3}+249018 \nu^{2}-131059 \nu+20598}{18240\left(4 \nu^{3}-10 \nu^{2}+6 \nu-1\right)} x-0.4196774909106648 x^{2}$,

$\rho_{82}(x ; \nu)=1+\frac{3063 \nu^{4}-22845 \nu^{3}+37119 \nu^{2}-17598 \nu+2462}{2736\left(7 \nu^{3}-14 \nu^{2}+7 \nu-1\right)} x-0.2261796441029474 x^{2}$

$\rho_{81}(x ; \nu)=1+\frac{21640 \nu^{4}-138430 \nu^{3}+236922 \nu^{2}-126451 \nu+20022}{18240\left(4 \nu^{3}-10 \nu^{2}+6 \nu-1\right)} x-0.26842133517043704 x^{2}$ 
The "eulerlog" functions euler $\log _{m}(x)$ are defined as

$$
\text { eulerlog } \log _{m}(x)=\gamma_{E}+\log 2+\frac{1}{2} \log x+\log m
$$

where $\gamma_{E}$ is Euler's constant, $\gamma_{E}=0.577215 \ldots$ and $\log (x)$ the natural logarithm function.

Let us now give the explicit expression of the residual phase corrections $\delta_{\ell m}$ that are implemented in the code. For $\delta_{2 m}, \delta_{33}$ and $\delta_{31}$ we list here explicitly both their Taylor-expanded forms (labeled with a "Taylor" superscript) and their Padé resummed ones. The $\delta_{\ell m}$ for higher multipoles can be given only in Taylor-expanded form and thus the label "Taylor" is omitted. The terms in boldface are the highest-order known $\mathrm{PN}$ terms for $\nu=0$. They are omitted when $\nu \neq 0$, and in particular in the computation of the Padé approximants, but they are kept in the computation of the $\nu=0$ EOB waveform. The Taylor-expanded $\delta_{\ell m}$ read

$$
\begin{aligned}
\delta_{22}^{\text {Taylor }}= & \frac{7}{3} y^{3 / 2}-24 \nu y^{5 / 2}+\frac{428}{105} \pi y^{3}\left(\frac{30995}{1134} \nu+\frac{962}{135} \nu^{2}\right) y^{7 / 2}+\left(\frac{\mathbf{1 7 1 2}}{\mathbf{3 1 5}} \boldsymbol{\pi}^{\mathbf{2}}-\frac{\mathbf{2 2 0 3}}{\mathbf{8 1}}\right) \boldsymbol{y}^{\mathbf{9} / \mathbf{2}}, \\
\delta_{21}^{\text {Taylor }}= & \frac{2}{3} y^{3 / 2}-\frac{493}{4} \nu y^{5 / 2}+\frac{107}{105} \pi y^{3}+\left(\frac{\mathbf{2 1 4}}{\mathbf{3 1 5}} \boldsymbol{\pi}^{\mathbf{2}}-\frac{\mathbf{2 7 2}}{\mathbf{8 1}}\right) \boldsymbol{y}^{\mathbf{9} / \mathbf{2}}, \\
\delta_{33}^{\text {Taylor }}= & \frac{13}{10} y^{3 / 2}-\frac{80897}{2430} \nu y^{5 / 2}+\frac{39}{7} \pi y^{3}+\left(\frac{\mathbf{7 8}}{\mathbf{7}} \boldsymbol{\pi}^{\mathbf{2}}-\frac{\mathbf{2 2 7 8 2 7}}{\mathbf{3 0 0 0}}\right) \boldsymbol{y}^{\mathbf{9} / \mathbf{2}}, \\
\delta_{32}= & \frac{10+33 \nu}{15(1-3 \nu)} y^{3 / 2}+\frac{52}{21} \pi y^{3}+\left(\frac{\mathbf{2 0 8}}{\mathbf{6 3}} \boldsymbol{\pi}^{\mathbf{2}}-\frac{\mathbf{9 1 1 2}}{\mathbf{4 0 5}}\right) \boldsymbol{y}^{\mathbf{9} / \mathbf{2}}, \\
\delta_{31}^{\text {Taylor }}= & \frac{13}{30} y^{3 / 2}-\frac{17 \nu}{10} y^{5 / 2}+\frac{13}{21} \pi y^{3}+\left(\frac{\mathbf{2 6}}{\mathbf{6 3}} \pi^{\mathbf{2}}-\frac{\mathbf{2 2 7 8 2 7}}{\mathbf{8 1 0 0 0}}\right) \boldsymbol{y}^{\mathbf{9} / \mathbf{2}}, \\
\delta_{44}= & \frac{112+219 \nu}{120(1-3 \nu)} y^{3 / 2}+\frac{25136}{3465} \pi y^{3}+\left(\frac{\mathbf{2 0 1 0 8 8}}{\mathbf{1 0 3 9 5}} \boldsymbol{\pi}^{\mathbf{2}}-\frac{\mathbf{5 5 1 4 4}}{\mathbf{3 7 5}}\right) \boldsymbol{y}^{\mathbf{9} / \mathbf{2}}, \\
\delta_{43}= & \frac{486+4961 \nu}{810(1-2 \nu)} y^{3 / 2}+\frac{1571}{385} \pi y^{3}, \\
\delta_{42}= & \frac{7(1+6 \nu)}{15(1-3 \nu)} y^{3 / 2}+\frac{6284}{3465} \pi y^{3}+\left(\frac{\mathbf{2 5 1 3 6}}{\mathbf{1 0 3 9 5}} \boldsymbol{\pi}^{\mathbf{2}}-\frac{\mathbf{6 8 9 3}}{\mathbf{3 7 5}}\right) \boldsymbol{y}^{\mathbf{9} / \mathbf{2}}, \\
\delta_{41}= & \frac{2+507 \nu}{10(1-2 \nu)} y^{3 / 2}+\frac{1571}{3465} \pi y^{3}, \\
\delta_{55}= & \frac{96875+857528 \nu}{131250(1-2 \nu)} y^{3 / 2} \cdot
\end{aligned}
$$

Among these, we used $\delta_{32}, \delta_{4 m}$ and $\delta_{55}$ in their Taylorexpanded form indicated above. By contrast, for $\delta_{22}$, $\delta_{21}, \delta_{33}$ and $\delta_{31}$ we used (denoting $v_{y} \equiv \sqrt{y}$ ) the following Padé resummed expressions (see Section II B 1 for further details)

$$
\begin{aligned}
\delta_{22} & =\frac{7}{3} v_{y}^{3} \frac{808920 \nu \pi v_{y}+137388 \pi^{2} v_{y}^{2}+35 \nu^{2}\left(136080+(154975-1359276 \nu) v_{y}^{2}\right)}{808920 \nu \pi v_{y}+137388 \pi^{2} v_{y}^{2}+35 \nu^{2}\left(136080+(154975+40404 \nu) v_{y}^{2}\right)} \\
\delta_{21} & =\frac{2}{3} v_{y}^{3} \frac{69020 \nu+5992 \pi v_{y}}{5992 \pi v_{y}+2456 \nu\left(28+493 \nu v_{y}^{2}\right)}, \\
\delta_{33}= & \frac{13}{10} v_{y}^{3} \frac{1+94770 \pi v_{y} /(566279 \nu)}{1+94770 \pi v_{y} /(566279 \nu)+80897 \nu v_{y}^{2} / 3159}, \\
\delta_{31}= & \frac{13}{30} v_{y}^{3} \frac{4641 \nu+1690 \pi v_{y}}{4641 \nu+1690 \pi v_{y}+18207 v_{y}^{2} \nu^{2}} .
\end{aligned}
$$


[1] A. Buonanno and T. Damour, Phys. Rev. D59, 084006 (1999)

[2] A. Buonanno and T. Damour, Phys. Rev. D62, 064015 (2000)

[3] T. Damour, P. Jaranowski, and G. Schaefer, Phys. Rev. D62, 084011 (2000)

[4] T. Damour, Phys. Rev. D64, 124013 (2001)

[5] T. Damour, B. R. Iyer, and A. Nagar, Phys. Rev. D79, 064004 (2009)

[6] T. Damour and A. Nagar, The Effective One Body description of the Two-Body problem, in Mass and Motion in General Relativity, edited by L. Blanchet, A. Spallicci and B. Whiting, Springer, Dordrecht (2011) pp. 211-252, arXiv:0906.1769 [gr-qc]

[7] T. Damour(2012), arXiv:1212.3169 [gr-qc]

[8] T. Damour and A. Nagar, Phys. Rev. D76, 064028 (2007), arXiv:0705.2519 [gr-qc]

[9] T. Damour and A. Nagar, Phys.Rev. D77, 024043 (2008), arXiv:0711.2628 [gr-qc]

[10] A. Buonanno, Y. Chen, and T. Damour, Phys. Rev. D74, 104005 (2006), arXiv:gr-qc/0508067

[11] T. Damour, P. Jaranowski, and G. Schaefer, Phys.Rev. D78, 024009 (2008), arXiv:0803.0915 [gr-qc]

[12] E. Barausse and A. Buonanno, Phys.Rev. D81, 084024 (2010), arXiv:0912.3517 [gr-qc]

[13] A. Nagar, Phys.Rev. D84, 084028 (2011), arXiv:1106.4349 [gr-qc]

[14] E. Barausse and A. Buonanno, Phys.Rev. D84, 104027 (2011), arXiv:1107.2904 [gr-qc]

[15] T. Damour and A. Nagar, Phys.Rev. D81, 084016 (2010), arXiv:0911.5041 [gr-qc]

[16] D. Bini, T. Damour, and G. Faye, Phys.Rev. D85, 124034 (2012), arXiv:1202.3565 [gr-qc]

[17] T. Damour, E. Gourgoulhon, and P. Grandclement, Phys.Rev. D66, 024007 (2002), arXiv:gr-qc/0204011 [grqc]

[18] A. Buonanno, G. B. Cook, and F. Pretorius, Phys.Rev. D75, 124018 (2007), arXiv:gr-qc/0610122 [gr-qc]

[19] A. Buonanno, Y. Pan, J. G. Baker, J. Centrella, B. J. Kelly, et al., Phys.Rev. D76, 104049 (2007), arXiv:0706.3732 [gr-qc]

[20] T. Damour, A. Nagar, E. N. Dorband, D. Pollney, and L. Rezzolla, Phys.Rev. D77, 084017 (2008), arXiv:0712.3003 [gr-qc]

[21] T. Damour, A. Nagar, M. Hannam, S. Husa, and B. Bruegmann, Phys.Rev. D78, 044039 (2008), arXiv:0803.3162 [gr-qc]

[22] T. Damour and A. Nagar, Phys. Rev. D79, 081503 (2009)

[23] A. Buonanno, Y. Pan, H. P. Pfeiffer, M. A. Scheel, L. T. Buchman, et al., Phys.Rev. D79, 124028 (2009), arXiv:0902.0790 [gr-qc]

[24] L. Baiotti, T. Damour, B. Giacomazzo, A. Nagar, and L. Rezzolla, Phys.Rev.Lett. 105, 261101 (2010), arXiv:1009.0521 [gr-qc]

[25] L. Baiotti, T. Damour, B. Giacomazzo, A. Nagar, and L. Rezzolla, Phys.Rev. D84, 024017 (2011), arXiv:1103.3874 [gr-qc]

[26] T. Damour, A. Nagar, D. Pollney, and C. Reisswig, Phys.Rev.Lett. 108, 131101 (2012), 4 pages, 2 figures, arXiv:1110.2938 [gr-qc]
[27] S. Bernuzzi, A. Nagar, M. Thierfelder, and B. Brugmann, Phys.Rev. D86, 044030 (2012), arXiv:1205.3403 [gr-qc]

[28] Y. Pan, A. Buonanno, M. Boyle, L. T. Buchman, L. E. Kidder, et al., Phys.Rev. D84, 124052 (2011), 26 pages, 25 figures, published Phys. Rev. D version, arXiv:1106.1021 [gr-qc]

[29] A. Taracchini, Y. Pan, A. Buonanno, E. Barausse, M. Boyle, et al., Phys.Rev. D86, 024011 (2012), arXiv:1202.0790 [gr-qc]

[30] L. Blanchet, Living Rev.Rel. 9, 4 (2006)

[31] T. Damour, A. Nagar, and L. Villain, Phys.Rev. D85, 123007 (2012), arXiv:1203.4352 [gr-qc]

[32] D. A. Brown, P. Kumar, and A. H. Nitz(2012), arXiv:1211.6184 [gr-qc]

[33] A. Le Tiec, A. H. Mroue, L. Barack, A. Buonanno, H. P. Pfeiffer, et al., Phys.Rev.Lett. 107, 141101 (2011), arXiv:1106.3278 [gr-qc]

[34] L. T. Buchman, H. P. Pfeiffer, M. A. Scheel, and B. Szilagyi, Phys.Rev. D86, 084033 (2012), arXiv:1206.3015 [gr-qc]

[35] T. Damour, Phys.Rev. D81, 024017 (2010), arXiv:0910.5533 [gr-qc]

[36] L. Blanchet, S. L. Detweiler, A. Le Tiec, and B. F. Whiting, Phys.Rev. D81, 084033 (2010), arXiv:1002.0726 [grqc]

[37] T. Damour, (unpublished); cited in Ref. [46], which quoted and used some combinations of the logarithmic contributions to $a(u)$ and $\bar{d}(u)(2010)$

[38] E. Barausse, A. Buonanno, and A. Le Tiec, Phys.Rev. D85, 064010 (2012), arXiv:1111.5610 [gr-qc]

[39] L. Blanchet, S. Detweiler, A. Le Tiec, and B. F. Whiting, Fundam.Theor.Phys. 162, 415 (2011), arXiv:1007.2614 [gr-qc]

[40] A. Le Tiec, E. Barausse, and A. Buonanno, Phys.Rev.Lett. 108, 131103 (2012), arXiv:1111.5609 [gr-qc]

[41] A. Nagar and S. Akcay, Phys.Rev. D85, 044025 (2012), arXiv:1112.2840 [gr-qc]

[42] S. Bernuzzi, A. Nagar, and A. Zenginoglu, Phys.Rev. D86, 104038 (2012), arXiv:1207.0769 [gr-qc]

[43] D. Bini and T. Damour, Phys.Rev. D86, 124012 (2012), arXiv:1210.2834 [gr-qc]

[44] G. Faye, S. Marsat, L. Blanchet, and B. R. Iyer, Class.Quant.Grav. 29, 175004 (2012), arXiv:1204.1043 [gr-qc]

[45] Y. Pan, A. Buonanno, L. T. Buchman, T. Chu, L. E. Kidder, et al., Phys.Rev. D81, 084041 (2010), arXiv:0912.3466 [gr-qc]

[46] L. Barack, T. Damour, and N. Sago, Phys.Rev. D82, 084036 (2010), arXiv:1008.0935 [gr-qc]

[47] A. Le Tiec, L. Blanchet, and B. F. Whiting, Phys.Rev. D85, 064039 (2012), arXiv:1111.5378 [gr-qc]

[48] S. Akcay, L. Barack, T. Damour, and N. Sago, Phys.Rev. D86, 104041 (2012), arXiv:1209.0964 [gr-qc]

[49] L. Barack and N. Sago, Phys.Rev.Lett. 102, 191101 (2009), arXiv:0902.0573 [gr-qc]

[50] T. Damour and A. Gopakumar, Phys. Rev. D73, 124006 (2006), arXiv:gr-qc/0602117

[51] R. Fujita and B. R. Iyer, Phys. Rev. D82, 044051 (2010), arXiv:1005.2266 [gr-qc] 
[52] T. Damour, A. Nagar, and M. Trias, Phys.Rev. D83, 024006 (2011), arXiv:1009.5998 [gr-qc]

[53] R. Fujita, Prog.Theor.Phys. 127, 583 (2012), arXiv:1104.5615 [gr-qc]

[54] R. Fujita, Prog.Theor.Phys. 128, 971 (2012), arXiv:1211.5535 [gr-qc]

[55] S. Bernuzzi, A. Nagar, and A. Zenginoglu, Phys.Rev. D83, 064010 (2011), arXiv:1012.2456 [gr-qc]

[56] E. Barausse, A. Buonanno, S. A. Hughes, G. Khanna, S. O'Sullivan, et al., Phys.Rev. D85, 024046 (2012), arXiv:1110.3081 [gr-qc]

[57] E. Berti, V. Cardoso, and C. M. Will, Phys.Rev. D73, 064030 (2006), arXiv:gr-qc/0512160 [gr-qc]

[58] E. Poisson and M. Sasaki, Phys.Rev. D51, 5753 (1995), arXiv:gr-qc/9412027 [gr-qc]

[59] K. Alvi, Phys.Rev. D64, 104020 (2001), arXiv:grqc/0107080 [gr-qc]

[60] B. R. Iyer and C. Will, Phys.Rev. D52, 6882 (1995)

[61] A. Gopakumar, B. R. Iyer, and S. Iyer, Phys.Rev. D55, 6030 (1997), arXiv:gr-qc/9703075 [gr-qc]

[62] A. Gopakumar and B. R. Iyer, Phys.Rev. D56, 7708 (1997), arXiv:gr-qc/9710075 [gr-qc]

[63] T. Damour and A. Nagar, Phys.Rev. D76, 044003 (2007), arXiv:0704.3550 [gr-qc]

[64] M. A. Scheel, H. P. Pfeiffer, L. Lindblom, L. E. Kidder, O. Rinne, et al., Phys.Rev. D74, 104006 (2006), arXiv:grqc/0607056 [gr-qc]

[65] M. Boyle, L. Lindblom, H. Pfeiffer, M. Scheel, and L. E. Kidder, Phys.Rev. D75, 024006 (2007), arXiv:grqc/0609047 [gr-qc]
[66] M. Boyle, D. A. Brown, L. E. Kidder, A. H. Mroue, H. P. Pfeiffer, et al., Phys.Rev. D76, 124038 (2007), arXiv:0710.0158 [gr-qc]

[67] M. Boyle, A. Buonanno, L. E. Kidder, A. H. Mroue, Y. Pan, et al., Phys.Rev. D78, 104020 (2008), arXiv:0804.4184 [gr-qc]

[68] M. A. Scheel et al., Phys. Rev. D79, 024003 (2009), arXiv:0810.1767 [gr-qc]

[69] I. MacDonald, A. H. Mroue, H. P. Pfeiffer, M. Boyle, L. E. Kidder, et al., Phys.Rev. D87, 024009 (2013), arXiv:1210.3007 [gr-qc]

[70] A. M. Abrahams and R. H. Price, Phys.Rev. D53, 1963 (1996), arXiv:gr-qc/9508059 [gr-qc]

[71] E. Pazos, E. N. Dorband, A. Nagar, C. Palenzuela, E. Schnetter, et al., Class.Quant.Grav. 24, S341 (2007), arXiv:gr-qc/0612149 [gr-qc]

[72] L. Baiotti, S. Bernuzzi, G. Corvino, R. De Pietri, and A. Nagar, Phys.Rev. D79, 024002 (2009), arXiv:0808.4002 [gr-qc]

[73] S. Bernuzzi and A. Nagar, Phys. Rev. D81, 084056 (2010), arXiv:1003.0597 [gr-qc]

[74] S. Bernuzzi, M. Thierfelder, and B. Bruegmann, Phys.Rev. D85, 104030 (2012), arXiv:1109.3611 [gr-qc]

[75] A. Zenginoglu, Class. Quant. Grav. 25, 145002 (2008), arXiv:0712.4333 [gr-qc]

[76] A. Zenginoglu, Class. Quant. Grav. 27, 045015 (2010), arXiv:0911.2450 [gr-qc]

[77] A. Zenginoglu, J.Comput.Phys. 230, 2286 (2011), arXiv:1008.3809 [math.NA] 